\title{
UNIFORM REGULARITY AND VANISHING VISCOSITY LIMIT FOR THE CHEMOTAXIS-NAVIER-STOKES SYSTEM IN A 3D BOUNDED DOMAIN
}

\author{
ZHIPENG ZHANG
}

\begin{abstract}
We investigate the uniform regularity and vanishing viscosity limit for the incompressible chemotaxis-Navier-Stokes system in a smooth bounded domain $\Omega \subset \mathbb{R}^{3}$. It is shown that there exists a unique strong solution of the incompressible chemotaxis-Navier-Stokes system in a finite time interval which is independent of the viscosity coefficient. Moreover, the solution is uniformly bounded in a conormal Sobolev space, which allows us to take the vanishing viscosity limit to obtain the incompressible inviscid chemotaxis-Navier-Stokes system.
\end{abstract}

\section{INTRODUCTION}

Chemotaxis is a biological process in which cells or bacteria move towards a chemically more favorable environment. For example, bacteria move towards higher concentration of oxygen which they consume. A typical model describing chemotaxis is the Keller-Segel equations derived by Keller and Segel in [13. which have been studied extensively. In nature, bacteria often live in a viscous fluid so that a convective transport of both cells and chemicals is happened through the fluid, and meanwhile a gravitation effect on the motion of the fluid is produced by the heavier bacteria. Thus, this interaction become more complicated since we not only pay attention to chemotaxis and diffusion but also transport and fluid dynamics. To describe the above biological phenomena, Tuval et al in 29] proposed the following model

$$
\begin{aligned}
& n_{t}+u \cdot \nabla n=\epsilon_{1} \Delta n-\nabla \cdot(k(c) n \nabla c), \\
& c_{t}+u \cdot \nabla c=\epsilon_{2} \Delta c-f(c) n, \\
& u_{t}+u \cdot \nabla u+\nabla p=\epsilon_{3} \Delta u-n \nabla \phi, \\
& \nabla \cdot u=0
\end{aligned}
$$

in $(0, T) \times \Omega$. The unknowns in (1.1) -(1.4) are $n(t, x), c(t, x), u(t, x)$ and $p(t, x)$, denoting the cell density, chemical concentration, velocity field and pressure of the

Date: November 9, 2018.

2000 Mathematics Subject Classification. 35Q30, 76D03, 76D05, 76D07.

Key words and phrases. Incompressible chemotaxis-Navier-Stokes system, Conormal Sobolev space, Vanishing viscosity limit, Navier boundary conditions. 
fluid, respectively. The pressure $p(t, x)$ in (1.3) can be recovered from $n$ and $u$ via an explicit Caldern-Zygmund singular integral operator [3]. The nonnegative functions $f(c)$ and $k(c)$ denote the chemical consumption rate and chemotaxis sensitivity. The given function $\phi$ represents the potential function produced by different physical mechanism, such as the gravitational force or centrifugal force. $\epsilon_{i}(i=1,2)$ are the corresponding diffusion coefficients for the cells and chemicals, and $\epsilon_{3}$ is the viscous coefficient for the fluid.

Due to the significance of the biological background, this model has been studied extensively and the main focus is on the solvability, see $[1,5,12,17,19,23,24$ and the references cited therein. Especially, Lorz [1] showed the local existence of weak solution for the above model in three bounded domain. Duan, Lorz and Markowich 24. obtained the global existence of the solution of the system (1.1)-(1.4) and the time decay rates of the classical solution near constant states in $\mathbb{R}^{3}$. In [17, Chae, Kang, and Lee proved the local well-posedness and blow up criterion of the smooth solution for the chemotaxis-Navier-Stokes system in $\mathbb{R}^{d}(d=2,3)$ and the global existence of the classical solution in $\mathbb{R}^{2}$ under some assumptions on the consumption rate and the chemotaxis sensitivity.

However, the research on the uniform regularity and vanishing viscosity limit for the system (1.1)-(1.4) is very limited. To the best of knowledge of the author, the only result is given by Zhang 22. He proved the the inviscid limit of the 3D chemotaxis-Navier-Stokes system in the whole space and established the convergence rate. From the biological point of review, it is more interesting to study this problem in a bounded domain.

The purpose of this paper is to investigate the uniform regularity and vanishing viscosity limit for the following chemotaxis-Navier-Stokes system

$$
\begin{aligned}
& n_{t}^{\epsilon}+u^{\epsilon} \cdot \nabla n^{\epsilon}=\Delta n^{\epsilon}-\nabla \cdot\left(n^{\epsilon} \nabla c^{\epsilon}\right), \\
& c_{t}^{\epsilon}+u^{\epsilon} \cdot \nabla c^{\epsilon}=\Delta c^{\epsilon}-c^{\epsilon} n^{\epsilon}, \\
& u_{t}^{\epsilon}+u^{\epsilon} \cdot \nabla u^{\epsilon}+\nabla p^{\epsilon}=\epsilon \Delta u^{\epsilon}-n^{\epsilon} \nabla \phi, \\
& \nabla \cdot u^{\epsilon}=0,
\end{aligned}
$$

in $(0, T) \times \Omega$. Here, $\Omega$ is a smooth bounded domain of $\mathbb{R}^{3}$. The chemotaxis-NavierStokes system (1.5)-(1.8) is considered under the initial condition

$$
\left.\left(n^{\epsilon}, c^{\epsilon}, u^{\epsilon}\right)\right|_{t=0}=\left(n_{0}^{\epsilon}, c_{0}^{\epsilon}, u_{0}^{\epsilon}\right)
$$

and the homogeneous boundary condition of Neumann type for $n^{\epsilon}$ and $c^{\epsilon}$

$$
\frac{\partial n^{\epsilon}}{\partial \nu}=\frac{\partial c^{\epsilon}}{\partial \nu}=0
$$

where $\nu$ stands for the outward unit normal vector to $\Omega$, and the Navier boundary condition for $u^{\epsilon}$ as

$$
u^{\epsilon} \cdot \nu=0, \quad\left(S u^{\epsilon} \cdot \nu\right)_{\tau}=-\zeta u_{\tau}^{\epsilon} \quad \text { on } \partial \Omega,
$$


where $\zeta$ is a coefficient measuring the tendency of the fluid to slip on the boundary, $S$ is the strain tensor defined by

$$
S u^{\epsilon}=\frac{1}{2}\left(\nabla u^{\epsilon}+\left(\nabla u^{\epsilon}\right)^{t}\right),
$$

$\left(\nabla u^{\epsilon}\right)^{t}$ denotes the transpose of the matrix $\nabla u^{\epsilon}$, and $u_{\tau}^{\epsilon}$ stands for the tangential part of $u^{\epsilon}$ on $\partial \Omega$, i.e.

$$
u_{\tau}^{\epsilon}=u^{\epsilon}-\left(u^{\epsilon} \cdot \nu\right) \nu
$$

The boundary condition (1.11) was introduced by Navier in 20. to show that the velocity is propositional to the tangential part of the stress. It allow the fluid slip along the boundary and is often used to model rough boundaries.

We point out that when $n^{\epsilon}=c^{\epsilon}=0$ in the system (1.5)-(1.8), it is reduced to the classical incompressible Navier-Stokes equations

$$
\begin{aligned}
& u_{t}^{\epsilon}+u^{\epsilon} \cdot \nabla u^{\epsilon}+\nabla p^{\epsilon}=\epsilon \Delta u^{\epsilon}, \\
& \nabla \cdot u^{\epsilon}=0 .
\end{aligned}
$$

There are lots of results on the inviscid limit to the incompressible Navier-Stokes equations, see $[2,8,11,14,16,18,21,26,28,32]$ and the references therein. When the incompressible Navier-Stokes equations (1.12)-(1.13) are supplemented with the boundary condition

$$
u^{\epsilon} \cdot \nu=0, \quad \nu \times \omega_{u}^{\epsilon}=0 \quad \text { on } \partial \Omega,
$$

where $\omega_{u}^{\epsilon}=\nabla \times u^{\epsilon}$, Xiao and Xin [32] obtained the local existence of strong solution with some uniform bounds in $H^{3}(\Omega)$ and the vanishing viscosity limit. Subsequently, their result was extended to $W^{k, p}(\Omega)$ in $[8]$. The main reason is that the boundary integrals vanishes on flat portions of the boundary, see also [9, 10, Later, the results in [8,32] were generalized by Berselli and Spirito [2] to a general bounded domain under certain restrictions on the initial data. Recently, Masmoudi and Rousset [18] considered the uniform regularity and vanishing viscosity limit for the incompressible Navier-Stokes equations (1.12)-(1.13) with the Navier boundary condition (1.11) in the anisotropic conormal Sobolev spaces which will be defined below.

Motivated by the ideas of [18, in this paper, we investigate the uniform regularity of the solution to the problem (1.5)-(1.11) in the anisotropic conormal Sobolev spaces and take the inviscid limit $\epsilon \rightarrow 0$ to obtain the following limit system (Assume that $\left(n^{\epsilon}, c^{\epsilon}, u^{\epsilon}\right)$ converge to $\left(n^{0}, c^{0}, u^{0}\right)$ in some sense.)

$$
\begin{aligned}
& n_{t}^{0}+u \cdot \nabla n^{0}=\Delta n^{0}-\nabla \cdot\left(n^{0} \nabla c^{0}\right), \\
& c_{t}^{0}+u \cdot \nabla c^{0}=\Delta c^{0}-c^{0} n^{0}, \\
& u_{t}^{0}+u \cdot \nabla u^{0}+\nabla p^{0}=-n^{0} \nabla \phi, \\
& \nabla \cdot u^{0}=0,
\end{aligned}
$$


in $(0, T) \times \Omega$ with the initial and boundary conditions

$$
\begin{aligned}
& \left.\left(n^{0}, c^{0}, u^{0}\right)\right|_{t=0}=\left(n_{0}, c_{0}, u_{0}\right), \\
& u^{0} \cdot \nu=0, \quad \frac{\partial n^{0}}{\partial \nu}=\frac{\partial c^{0}}{\partial \nu}=0 \quad \text { on } \quad \partial \Omega .
\end{aligned}
$$

Before stating our main results, we first introduce the notations and conventions used throughout this paper. We assume that $\Omega$ has a covering such that

$$
\Omega \subset \Omega_{0} \cup_{k=1}^{n} \Omega_{k},
$$

where $\overline{\Omega_{0}} \subset \Omega$ and in each $\Omega_{k}$ there exists a function $\psi_{k}$ such that

$$
\begin{aligned}
\Omega \cup \Omega_{k} & =\left\{x=\left(x_{1}, x_{2}, x_{3}\right) \mid x_{3}>\psi_{k}\left(x_{1}, x_{2}\right)\right\} \cup \Omega_{k}, \\
\partial \Omega \cup \Omega_{k} & =\left\{x=\left(x_{1}, x_{2}, x_{3}\right) \mid x_{3}=\psi_{k}\left(x_{1}, x_{2}\right)\right\} \cup \Omega_{k} .
\end{aligned}
$$

We say that $\Omega$ is $\mathcal{C}^{m}$ if the functions $\psi_{k}$ are $\mathcal{C}^{m}$-functions.

To define the conormal Sobolev spaces, we consider $\left(Z_{k}\right)_{1 \leq k \leq N}$, a finite set of generators of vector fields that are tangent to $\partial \Omega$, and set

$$
H_{c o}^{m}(\Omega):=\left\{f \in L^{2}(\Omega) \mid Z^{I} f \in L^{2}(\Omega) \text { for }|I| \leq m, m \in \mathbb{N}\right\},
$$

where $I=\left(k_{1}, \ldots, k_{m}\right), Z^{I}:=Z_{k_{1}} \cdots Z_{k_{m}}$. We define the norm of $H_{c o}^{m}(\Omega)$ as

$$
\|f\|_{m}^{2}:=\sum_{|I| \leq m}\left\|Z^{I} f\right\|_{L^{2}}^{2}
$$

We say a vector field, $u$, is in $H_{c o}^{m}(\Omega)$ if each of its components is in $H_{c o}^{m}(\Omega)$ and

$$
\|u\|_{m}^{2}:=\sum_{i=1}^{3} \sum_{|I| \leq m}\left\|Z^{I} u_{i}\right\|_{L^{2}}^{2}
$$

is finite. In the same way, we set

$$
\begin{aligned}
\|f\|_{m, \infty} & :=\sum_{|I| \leq m}\left\|Z^{I} f\right\|_{L^{\infty}}, \\
\left\|\nabla Z^{m} f\right\|^{2} & :=\sum_{|I|=m}\left\|\nabla Z^{I} f\right\|_{L^{2}}^{2},
\end{aligned}
$$

and we say that $f \in W_{c o}^{m, \infty}(\Omega)$ if $\|f\|_{m, \infty}$ is finite. By using the above covering of $\Omega$, we can assume that each vector field is supported in one of $\left\{\Omega_{i}\right\}_{i=0}^{n}$. Also, we note that the $\|\cdot\|_{m}$ norm yields a control of the standard $H^{m}$ norm in $\Omega_{0}$, whereas if $\Omega_{i} \cap \partial \Omega \neq \emptyset$, there is no control of the normal derivatives.

Since $\partial \Omega$ is given locally by $x_{3}=\psi\left(x_{1}, x_{2}\right)$ (We omit the subscript $k$ for notational convenience), it is convenient to use the coordinates:

$$
\Psi:(y, z) \mapsto(y, \psi(y)+z)=x .
$$

A local basis is thus given by the vector fields $\left(\partial_{y^{1}}, \partial_{y^{1}}, \partial_{z}\right)$ where $\partial_{y^{1}}$ and $\partial_{y^{2}}$ are tangent to $\partial \Omega$ on the boundary and in general $\partial_{z}$ is usually not a normal vector field. We sometimes use the notation $\partial_{y^{3}}$ for $\partial_{z}$. By using this parametrization, we 
can take suitable vector fields compactly supported in $\Omega_{i}$ in the definition of the $\|\cdot\|_{m}$ norms:

$$
Z_{i}=\partial_{y^{i}}=\partial_{i}+\partial_{i} \psi \partial_{z}, i=1,2, \quad Z_{3}=\varphi(z) \partial_{z},
$$

where $\varphi(z)=\frac{z}{1+z}$ is a smooth and supported function in $[0,+\infty)$ and satisfies

$$
\varphi(0)=0, \varphi^{\prime}(0)>0, \varphi(z)>0 \text { for } z>0 .
$$

In this paper, we shall still denote by $\partial_{i}, i=1,2,3$ or $\nabla$ the derivatives with respect to the standard coordinates of $\mathbb{R}^{3}$. The coordinates of a vector field $u$ in the basis $\left(\partial_{y^{1}}, \partial_{y^{1}}, \partial_{z}\right)$ will be denote by $u^{i}$, thus

$$
u=u^{1} \partial_{y^{1}}+u^{2} \partial_{y^{2}}+u^{3} \partial_{z} .
$$

We denote by $u_{i}$ the coordinates in the standard basis of $\mathbb{R}^{3}$, i.e.

$$
u=u_{1} \partial_{1}+u_{2} \partial_{2}+u_{3} \partial_{3} .
$$

The unit outward normal vector $\nu$ is given locally by

$\nu(x)=\nu(\Psi(y, z)):=\frac{1}{\sqrt{1+|\nabla \psi(y)|^{2}}}\left(\begin{array}{c}\partial_{1} \psi(y) \\ \partial_{2} \psi(y) \\ -1\end{array}\right), \quad N(x):=\sqrt{1+|\nabla \psi(y)|^{2}} \nu(x)$

and denote by $\Pi$ the orthogonal projection

$$
\Pi(x) u=\Pi(\Psi(y, z)) u:=u-[u \cdot \nu(\Psi(y, z))] \nu(\Psi(y, z)
$$

which gives the orthogonal projector onto the tangent space of the boundary. Note that both $\nu$ and $\Pi$ are defined in the whole $\Omega_{k}$ and do not depend on $z$. By using these notations, the Navier boundary condition (1.11) reads

$$
u^{\epsilon} \cdot \nu=0, \quad \Pi \partial_{\nu} u^{\epsilon}=\theta\left(u^{\epsilon}\right)-2 \zeta \Pi u^{\epsilon},
$$

where $\theta$ is the shape operator (second fundamental form) of the boundary,

$$
\theta\left(u^{\epsilon}\right):=\Pi\left((\nabla \nu) u^{\epsilon}\right) .
$$

For later use and notational convenience, we set

$$
\mathcal{Z}^{\alpha}=\partial_{t}^{\alpha_{0}} Z^{\alpha_{1}}=\partial_{t}^{\alpha_{0}} Z_{1}^{\alpha_{11}} Z_{2}^{\alpha_{12}} Z_{3}^{\alpha_{13}},
$$

and we also use the following notations

$$
\|f(t)\|_{\mathcal{H}^{m}}^{2}:=\sum_{|\alpha| \leq m}\left\|\mathcal{Z}^{\alpha} f(t)\right\|_{L_{x}^{2}}^{2}, \quad\|f(t)\|_{\mathcal{H}^{m, \infty}}^{2}:=\sum_{|\alpha| \leq m}\left\|\mathcal{Z}^{\alpha} f(t)\right\|_{L_{x}^{\infty}}^{2}
$$

for smooth time-space function $f(t, x)$.

Throughout the paper, we shall denote by $\|\cdot\|_{H^{m}}$ and $\|\cdot\|_{W^{m, \infty}}$ the standard Sobolev norms in $\Omega$ and the notation $|\cdot|_{H^{m}}$ will be used for the standard Sobolev norm of functions defined on $\partial \Omega$. Note that this norm involves only tangential derivatives. $\|\cdot\|$ stands for the standard $L^{2}$ norm and $(\cdot, \cdot)$ for the $L^{2}$ scalar 
product. The letter $D$ and $d$ are positive numbers which may change from line to line, but independent of $\epsilon \in(0,1]$. $D_{m}$ stands for a positive constant independent of $\epsilon$ which depends on the $\mathcal{C}^{m}$-norm of the functions $\psi_{k} . P(\cdot)$ denotes a polynomial function.

In order to obtain the uniform estimates for the solutions of the chemotaxisNavier-Stokes system with the boundary conditions (1.10) and (1.11), we need to find a suitable functional space. Here, we define the functional space $\mathcal{E}_{m}^{\epsilon}(T)$ for functions $\left(n^{\epsilon}, c^{\epsilon}, u^{\epsilon}\right)(t, x)$ as follows:

$$
\mathcal{E}_{m}^{\epsilon}(T)=\left\{\left(n^{\epsilon}, c^{\epsilon}, u^{\epsilon}\right) \in L^{\infty}\left([0, T], L^{2}\right) \mid \operatorname{esssup}_{0 \leq t \leq T}\left\|\left(n^{\epsilon}, c^{\epsilon}, u^{\epsilon}\right)(t)\right\|_{\mathcal{E}_{m}^{\epsilon}}<+\infty\right\}
$$

where the norms $\|(\cdot, \cdot, \cdot)\|_{\mathcal{E}_{m}^{\epsilon}}$ is given by

$$
\begin{aligned}
\left\|\left(n^{\epsilon}, c^{\epsilon}, u^{\epsilon}\right)(t)\right\|_{\mathcal{E}_{m}^{\epsilon}}:= & \left\|\left(n^{\epsilon}, c^{\epsilon}, u^{\epsilon}\right)\right\|_{\mathcal{H}^{m}}+\left\|\left(\nabla n^{\epsilon}, \nabla u^{\epsilon}\right)(t)\right\|_{\mathcal{H}^{m-1}}+\left\|\nabla c^{\epsilon}\right\|_{\mathcal{H}^{m}} \\
& +\left\|\left(\Delta n^{\epsilon}, \Delta c^{\epsilon}\right)\right\|_{\mathcal{H}^{m-1}}+\left\|\nabla u^{\epsilon}\right\|_{\mathcal{H}^{1, \infty}} .
\end{aligned}
$$

Correspondingly, for the initial data $\left(n_{0}^{\epsilon}, c_{0}^{\epsilon}, u_{0}^{\epsilon}\right)$, we difine

$$
\begin{aligned}
& \sup _{0<\epsilon \leq 1}\left\|\left(n_{0}^{\epsilon}, c_{0}^{\epsilon}, u_{0}^{\epsilon}\right)\right\|_{\mathcal{E}_{m}^{\epsilon}}:=\sup _{0<\epsilon \leq 1}\{\left\|\left(n_{0}^{\epsilon}, c_{0}^{\epsilon}, u_{0}^{\epsilon}\right)\right\|_{\mathcal{H}^{m}}+\left\|\left(\nabla n_{0}^{\epsilon}, \nabla u_{0}^{\epsilon}\right)\right\|_{\mathcal{H}^{m-1}}+\left\|\nabla c_{0}^{\epsilon}\right\|_{\mathcal{H}^{m}} \\
&\left.+\left\|\left(\Delta n_{0}^{\epsilon}, \Delta c_{0}^{\epsilon}\right)\right\|_{\mathcal{H}^{m-1}}+\left\|\nabla u_{0}^{\epsilon}\right\|_{\mathcal{H}^{1, \infty}}\right\} \leq \widetilde{D}_{0},
\end{aligned}
$$

where $\widetilde{D}_{0}$ is a positive constant independent of $\epsilon \in(0,1]$, and the time derivatives of initial data in (1.29) are defined through the system (1.5)-(1.8). Thus, the initial data $\left(n_{0}^{\epsilon}, c_{0}^{\epsilon}, u_{0}^{\epsilon}\right)$ is assumed to have a higher space regularity and compatibility. We note that the a priori estimates in Theorem 3.1 below are obtained in the situation that the approximate solution is sufficiently smooth up to the boundary. Therefore, in order to obtain a self-contained result, we need to assume the approximated initial data satisfies the boundary compatibility condition (1.11). For the initial data $\left(n_{0}^{\epsilon}, c_{0}^{\epsilon}, u_{0}^{\epsilon}\right)$ satisfying (1.29), it is not clear if there exists an approximate sequence $\left(n_{0}^{\epsilon, \delta}, c_{0}^{\epsilon, \delta}, u_{0}^{\epsilon, \delta}\right)$ ( $\delta$ being a regularization parameter), which satisfy the boundary compatibilities and $\left\|\left(n_{0}^{\epsilon, \delta}-n_{0}^{\epsilon}, c_{0}^{\epsilon, \delta}-c_{0}^{\epsilon}, u_{0}^{\epsilon, \delta}-u_{0}^{\epsilon}\right)\right\|_{\mathcal{E}_{m}^{\epsilon}} \rightarrow 0$ as $\delta \rightarrow 0$. Thus, we set

$$
\begin{aligned}
\mathcal{E}_{C N S, a p}^{m}:=\{ & \left(n^{\epsilon}, c^{\epsilon}, u^{\epsilon}\right) \in \mathcal{C}^{2 m+1} \times \mathcal{C}^{2 m+1} \times \mathcal{C}^{2 m} \mid \\
& \partial_{t}^{k} n^{\epsilon}, \partial_{t}^{k} c^{\epsilon}, \partial_{t}^{k} u^{\epsilon}, \partial_{t}^{k} \nabla n^{\epsilon}, \partial_{t}^{k} \nabla c^{\epsilon}, k=1, \ldots, m \text { are defined through } \\
& \text { the system (1.5) - (1.11) and } \partial_{t}^{k} u^{\epsilon}, \partial_{t}^{k} \nabla n^{\epsilon}, \partial_{t}^{k} \nabla c^{\epsilon}, k=1, \ldots, m-1 \\
& \text { satisfy the boundary compatibility condition }\}
\end{aligned}
$$

and

$$
\mathcal{E}_{C N S}^{m}:=\text { The closure of } \mathcal{E}_{C N S, a p}^{m, \epsilon} \text { in the norm }\|(\cdot, \cdot, \cdot)\|_{\mathcal{E}_{m}^{\epsilon}} .
$$

Our first result of this paper reads as follows: 
Theorem 1.1. Let $m$ be an integer satisfying $m \geq 6$ and $\Omega$ be a $\mathcal{C}^{m+2}$ domain. Assume that the initial data $\left(n_{0}^{\epsilon}, c_{0}^{\epsilon}, u_{0}^{\epsilon}\right) \in \mathcal{E}_{C N S}^{m}$ satisfy (1.29), $\nabla \cdot u_{0}^{\epsilon}=0$ and $\phi$ is a smooth function. Then, there exist $\widetilde{T}_{0}>0$ and $\widetilde{D}_{1}$, independent of $\epsilon \in(0,1]$ and $|\zeta| \leq 1$, such that there exists a unique solution of the problem (1.5)-(1.11) on $\left[0, \widetilde{T}_{0}\right]$ that satisfies

$$
\begin{aligned}
& \sup _{0 \leq \tau \leq t}\left(\left\|\left(n^{\epsilon}, c^{\epsilon}, u^{\epsilon}\right)\right\|_{\mathcal{H}^{m}}^{2}+\left\|\nabla\left(n^{\epsilon}, u^{\epsilon}\right)\right\|_{\mathcal{H}^{m-1}}^{2}+\left\|\nabla c^{\epsilon}\right\|_{\mathcal{H}^{m}}^{2}\right. \\
& \left.+\left\|\Delta\left(n^{\epsilon}, c^{\epsilon}\right)\right\|_{\mathcal{H}^{m-1}}^{2}+\left\|\nabla u^{\epsilon}\right\|_{1, \infty}^{2}\right)+\epsilon \int_{0}^{t}\left(\left\|\nabla u^{\epsilon}\right\|_{\mathcal{H}^{m}}^{2}+\left\|\nabla^{2} u^{\epsilon}\right\|_{\mathcal{H}^{m-1}}^{2}\right) d \tau \\
& +\int_{0}^{t}\left(\left\|\nabla n^{\epsilon}\right\|_{\mathcal{H}^{m}}^{2}+\left\|\Delta c^{\epsilon}\right\|_{\mathcal{H}^{m}}^{2}+\left\|\nabla \Delta\left(n^{\epsilon}, c^{\epsilon}\right)\right\|_{\mathcal{H}^{m-1}}^{2}\right) d \tau \leq \widetilde{D}_{1}, \quad \forall t \in\left[0, \widetilde{T}_{0}\right],
\end{aligned}
$$

where $\widetilde{D}_{1}$ depends only on $\widetilde{D}_{0}, D_{m+2}$ and $\phi$.

Remark 1.1. Since $\Delta c^{\epsilon}$ appear in the equation (1.5), we can not do the same higher order estimate for $\nabla n^{\epsilon}$ as $\nabla c^{\epsilon}$. Otherwise, we obtain the term $\left\|\nabla \Delta c^{\epsilon}\right\|_{\mathcal{H}^{m}}$ in the right-hand side of the energy inequality, which is out of control.

Remark 1.2. When the Navier boundary condition (1.11) is replaced by the following more generalized form

$$
u^{\epsilon} \cdot \nu=0, \quad \nu \times \omega_{u}^{\epsilon}=\left[B u^{\epsilon}\right]_{\tau} \quad \text { on } \quad \partial \Omega,
$$

where $B=2(A-S(\nu))$ and $A$ is a $(1,1)$-type tensor on the boundary $\partial \Omega$, we can still obtain the same results as those in Theorem 1.1.

Now we give some comments on the proof of Theorem 1.1. We shall follow and modify some ideas developed in [18. In fact, due to the strong coupling among $n^{\epsilon}$, $c^{\epsilon}$ and $u^{\epsilon}$, we need to overcome some new difficulties and to face more complicated energy estimates. The main step of the proof Theorem 1.1 can be stated as follows: First, we get a conormal energy estimates in $\mathcal{H}^{m}$ for $\left(n^{\epsilon}, c^{\epsilon}, u^{\epsilon}\right)$. The second step is to give the estimates for $\left\|\nabla n^{\epsilon}\right\|_{\mathcal{H}^{m-1}}$ and $\left\|\nabla c^{\epsilon}\right\|_{\mathcal{H}^{m}}$. Since the equation (1.5) involve the term $\Delta c^{\epsilon}$, we can not do the same higher order estimate for $\nabla n^{\epsilon}$ as $\nabla c^{\epsilon}$. In the third step, we show the estimates for $\left\|\Delta\left(n^{\epsilon}, c^{\epsilon}\right)\right\|_{\mathcal{H}^{m-1}}$. Since the dissipative terms $\Delta n^{\epsilon}$ and $\Delta c^{\epsilon}$ appear, we can easily deduce the estimates. Next, we focus on the estimate of $\left\|\nabla u^{\epsilon}\right\|_{\mathcal{H}^{m-1}}$ in the fourth step. In order to obtain this estimate by an energy method, $\partial_{\nu} u^{\epsilon}$ is not a convenient quantity, since it does not vanish on the boundary. However, due to the incompressible condition (1.8), $\left\|\partial_{\nu} u^{\epsilon} \cdot \nu\right\|_{\mathcal{H}^{m-1}}$ can be easily controlled by the $\mathcal{H}^{m}$ norm of $u^{\epsilon}$. Moreover, thank to the the Nvier boundary conditions (1.11), it is convenient to study $\eta^{\epsilon}=\left(S u^{\epsilon} \nu+\right.$ $\left.\zeta u^{\epsilon}\right)_{\tau}$. We find that $\eta^{\epsilon}$ satisfy equations with homogeneous Dirichlet boundary conditions and gives us control of $\left(\partial_{\nu} u^{\epsilon}\right)_{\tau}$, and by performing energy estimate on the equations solved by $\eta^{\epsilon}$, we can get a control of $\left\|\eta^{\epsilon}\right\|_{\mathcal{H}^{m-1}}$. The fifth step 
is to estimate the pressure. In the spirit of [18, we split the pressure into two parts which satisfy nonhomogeneous elliptic equations with Neumann boundary conditions. In view of the regularity theory of elliptic equations with Neumann boundary conditions, we get the estimates of the pressure terms. Finally, we need to estimate $\left\|u^{\epsilon}\right\|_{\mathcal{H}^{1, \infty}},\left\|\nabla\left(c^{\epsilon}, u^{\epsilon}\right)\right\|_{\mathcal{H}^{1, \infty}},\left\|\left(n^{\epsilon}, c^{\epsilon}\right)\right\|_{W^{2, \infty}}$ and $\left\|\nabla \Delta c^{\epsilon}\right\|_{L^{\infty}}$. By virtue of the anistropic Sobolev embedding inequality in Lemma 2.3 and the equations (1.5) and (1.6), we can give the estimates for them, except for $\left\|\nabla u^{\epsilon}\right\|_{\mathcal{H}^{1, \infty}}$. In order to estimate $\left\|\nabla u^{\epsilon}\right\|_{\mathcal{H}^{1, \infty}}$, similar to the fourth step, we find equivalent quantities $\widetilde{\eta}^{\epsilon}$ which satisfies a homogenous Dirichlet condition and solves a convection-diffusion equation. The estimate will be obtained by using Lemma 14 in [18].

Based on Theorem 1.1, we justify the vanishing viscosity limit as follows:

Theorem 1.2. Let $m$ be an integer satisfying $m \geq 6$ and $\Omega$ be a $\mathcal{C}^{m+2}$ domain. Consider $\left(n_{0}, c_{0}, u_{0}\right) \in \mathcal{E}_{C N S}^{m}$ and $\nabla \cdot u_{0}=0$ and $\left(n^{\epsilon}, c^{\epsilon}, u^{\epsilon}\right)$ the solution of the system (1.5)-(1.8) with the initial data $\left(n_{0}, c_{0}, u_{0}\right)$ and the boundary conditions (1.10) and (1.11) given by Theorem 1.1. Then, there exists a unique solution of the system (1.15)-(1.20) with initial value $\left(n_{0}, c_{0}, u_{0}\right),\left(n^{0}, c^{0}, u^{0}\right) \in \mathcal{E}_{C N S}^{m}$ such that

$$
\begin{aligned}
\sup _{0 \leq t \leq \widetilde{T}_{0}}( & \left\|u^{\epsilon}-u^{0}\right\|_{L^{2}}+\left\|\left(n^{\epsilon}, c^{\epsilon}\right)-\left(n^{0}, c^{0}\right)\right\|_{H^{1}} \\
& \left.+\left\|u^{\epsilon}-u^{0}\right\|_{L^{\infty}}+\left\|\left(n^{\epsilon}, c^{\epsilon}\right)-\left(n^{0}, c^{0}\right)\right\|_{W^{1, \infty}}\right) \rightarrow 0,
\end{aligned}
$$

when $\epsilon$ tends to zero.

The rest of the paper is organized as follows. In the following section, we present some inequalities that will be used frequently later. In Section 3, we prove a priori energy estimates. Next, we use the a priori estimates in Theorem 3.1 to give the proof of Theorem 1.1 in section 4. Finally, we prove Theorem 1.2 in Section 5 .

\section{Preliminaries}

We first introduce the Korn's inequlity which play an important role in energy estimates below.

Lemma 2.1 (Korn's inequality [4). Let $\Omega$ be a bounded Lipschitz domain of $\mathbb{R}^{3}$. There exists a constant $D>0$ depending only on $\Omega$ such that

$$
\|u\|_{H^{1}(\Omega)} \leq D\left(\|u\|_{L^{2}(\Omega)}+\|S(u)\|_{L^{2}(\Omega)}\right), \quad \forall u \in\left(H^{1}(\Omega)\right)^{3} .
$$

Next, we introduce the space

$$
\mathcal{W}^{m}([0, T] \times \Omega)=\left\{f(t, x) \in L^{2}([0, T] \times \Omega)\left|\mathcal{Z}^{\alpha} f \in L^{2}([0, T] \times \Omega),\right| \alpha \mid \leq m\right\} .
$$

Then, we have the following Gagliardo-Nirenberg-Moser type inequality whose proof can be found in [6]. 
Lemma 2.2. Let $u, v \in L^{\infty}([0, T] \times \Omega) \cap \mathcal{W}^{m}([0, T] \times \Omega)$, we have

$$
\begin{array}{r}
\int_{0}^{t}\left\|\mathcal{Z}^{\alpha_{1}} u \mathcal{Z}^{\alpha_{2}} v\right\|^{2} d \tau \leq D\left(\|u\|_{L_{t, x}^{\infty}}^{2} \int_{0}^{t}\|v\|_{\mathcal{H}^{m}}^{2} d \tau+\|v\|_{L_{t, x}^{\infty}}^{2} \int_{0}^{t}\|u\|_{\mathcal{H}^{m}}^{2} d \tau\right), \\
\left|\alpha_{1}\right|+\left|\alpha_{2}\right|=m .
\end{array}
$$

Finally, we need the following anistropic Sobolev embedding and trace estimates.

Lemma $2.3(18,30])$. Let $m_{1} \geq 0$ and $m_{2} \geq 0$ be integers, $u \in H_{c o}^{m_{1}}(\Omega) \cap H_{c o}^{m_{2}}(\Omega)$ and $\nabla u \in H_{c o}^{m_{2}}(\Omega)$. Then we have

$$
\begin{aligned}
& \|u\|_{L^{\infty}(\Omega)}^{2} \leq D\left(\|\nabla u\|_{m_{2}}+\|u\|_{m_{2}}\right)\|u\|_{m_{1}}, \quad m_{1}+m_{2} \geq 3, \\
& |u|_{H^{s}(\partial \Omega)}^{2} \leq D\left(\|\nabla u\|_{m_{2}}+\|u\|_{m_{2}}\right)\|u\|_{m_{1}}, \quad m_{1}+m_{2} \geq 2 s \geq 0 .
\end{aligned}
$$

\section{A priori estimates}

The main aim of this section is to prove the following a priori estimate which is the crucial step in the proof of Theorem 1.1. For notational convenience, we drop the superscript $\epsilon$ throughout this section.

Theorem 3.1. For $m>6$ and a $\mathcal{C}^{m+2}$ domain $\Omega$, there exists a constant $D_{m+2}>$ 0 , independent of $\epsilon \in(0,1]$ and $|\zeta| \leq 1$, such that for any sufficiently smooth solution defined on $[0, T]$ of the problem (1.5)-(1.11) in $\Omega$, we have

$$
\begin{aligned}
& \sup _{0 \leq \tau \leq t} N_{m}(\tau)+\epsilon \int_{0}^{t}\left(\|\nabla u\|_{\mathcal{H}^{m}}^{2}+\left\|\nabla^{2} u\right\|_{\mathcal{H}^{m-1}}^{2}\right) d \tau+\int_{0}^{t}\left(\|\nabla n\|_{\mathcal{H}^{m}}^{2}+\|\Delta c\|_{\mathcal{H}^{m}}^{2}\right. \\
& \left.+\|\nabla \Delta(n, c)\|_{\mathcal{H}^{m-1}}^{2}\right) d \tau \leq \widetilde{D}_{2} D_{m+2}\left\{N_{m}(0)+\left(1+P\left(N_{m}(t)\right)\right) \int_{0}^{t} P\left(N_{m}(\tau)\right) d \tau\right\},
\end{aligned}
$$

where $\widetilde{D}_{2}$ depends only on $\phi$ and

$$
N_{m}(t):=\|(n, c, u)\|_{\mathcal{H}^{m}}^{2}+\|\nabla(n, u)\|_{\mathcal{H}^{m-1}}^{2}+\|\nabla c\|_{\mathcal{H}^{m}}^{2}+\|\Delta(n, c)\|_{\mathcal{H}^{m-1}}^{2}+\|\nabla u\|_{1, \infty}^{2} .
$$

Since the proof of Theorem 3.1 is quite complicated and lengthy, we divided the proof into the following subsections.

3.1. Conormal Energy Estimates. In this subsection, we first give the basic $L^{2}$ energy estimates.

Lemma 3.1. For a smooth solution of the problem (1.5)-(1.11), we have

$$
\sup _{0 \leq \tau \leq t}\|u\|^{2}+\epsilon \int_{0}^{t}\|\nabla u\|^{2} d \tau \leq D_{2}\left(\left\|u_{0}\right\|^{2}+\int_{0}^{t}\left(\|u\|^{2}+\|n \nabla \phi\|^{2}\right) d \tau\right)
$$

for every $\epsilon \in(0,1]$ and $|\zeta| \leq 1$. 
Proof. Multiplying (1.7) by $u$, we obtain

$$
\left(u_{t}, u\right)+(u \cdot \nabla u, u)=\epsilon(\Delta u, u)-(\nabla p, u)-(n \nabla \phi, u) .
$$

Due to (1.8), (1.11) and integration by parts, we find

$$
\begin{aligned}
(\nabla p, u) & =\int_{\partial \Omega} p u \cdot \nu d \sigma-\int_{\Omega} p \nabla \cdot u d x=0, \\
(\epsilon \Delta u, u)=2 \epsilon(\nabla \cdot S u, u) & =-2 \epsilon\|S u\|^{2}+2 \epsilon \int_{\partial \Omega}((S u) \cdot \nu) \cdot u d \sigma \\
& =-2 \epsilon\|S u\|^{2}-2 \epsilon \zeta \int_{\partial \Omega}\left|u_{\tau}\right|^{2} d \sigma .
\end{aligned}
$$

From (3.4)-(3.6) and Lemma 2.1, we can obtain (3.3).

Next, we give the basic $L^{2}$ energy estimate for $(n, c)$.

Lemma 3.2. For a smooth solution of the problem (1.5)-(1.11), we have

$$
\sup _{0 \leq \tau \leq t}\|n\|^{2}+\int_{0}^{t}\|\nabla n\|^{2} d \tau \leq D_{2}\left(\left\|n_{0}\right\|^{2}+\int_{0}^{t}\|n \nabla c\|^{2} d \tau\right)
$$

for every $\epsilon \in(0,1]$ and $|\zeta| \leq 1$.

Proof. Multiplying (1.5) by $n$, we obtain

$$
\left(n_{t}, n\right)+(u \cdot \nabla n, n)=(\Delta n, n)-(\nabla \cdot(n \nabla c), n) .
$$

Due to (1.8)-(1.11) and integration by parts, we find

$$
(u \cdot \nabla n, n)=0, \quad(\Delta n, n)=-\|\nabla n\|^{2}, \quad(\nabla \cdot(n \nabla c), n)=-(n \nabla c, \nabla n) .
$$

Based on (3.9) and Young's inequality, we can obtain (3.7).

Similar to Lemma 3.2, we can easily get the following Lemma, the proof being omitted.

Lemma 3.3. For a smooth solution of the problem (1.5)-(1.11), we have

$$
\sup _{0 \leq \tau \leq t}\|c\|^{2}+\int_{0}^{t}\|\nabla c\|^{2} d \tau \leq D_{2}\left(\left\|c_{0}\right\|^{2}+\int_{0}^{t}\|\sqrt{n} c\|^{2} d \tau\right)
$$

for every $\epsilon \in(0,1]$ and $|\zeta| \leq 1$.

Now, we turn to the higher order energy estimates of $(n, c, u)$. Set

$$
M(t):=\sup _{0 \leq \tau \leq t}\left\{\|u\|_{\mathcal{H}^{1, \infty}}^{2}+\|\nabla(c, u)\|_{\mathcal{H}^{1, \infty}}^{2}+\|(n, c)\|_{W^{2, \infty}}^{2}+\|\nabla \Delta c\|_{L^{\infty}}^{2}\right\} .
$$

Lemma 3.4. For every $m \geq 0$, a smooth solution of the problem (1.5)-(1.11) satisfies the estimate

$$
\begin{aligned}
& \sup _{0 \leq \tau \leq t}\|u\|_{\mathcal{H}^{m}}^{2}+\epsilon \int_{0}^{t}\|\nabla u\|_{\mathcal{H}^{m}}^{2} d \tau \\
\leq & D_{m+2}\left\{\left\|u_{0}\right\|_{\mathcal{H}^{m}}^{2}+\int_{0}^{t}\left(\left\|\nabla^{2} p_{1}\right\|_{\mathcal{H}^{m-1}}\|u\|_{\mathcal{H}^{m}}+\epsilon^{-1}\left(\left\|\nabla p_{2}\right\|_{\mathcal{H}^{m-1}}^{2}+\left\|p_{2}\right\|_{\mathcal{H}^{m-1}}^{2}\right)\right) d \tau\right.
\end{aligned}
$$




$$
\left.+(1+P(M(t))) \int_{0}^{t}\left(\|u\|_{\mathcal{H}^{m}}^{2}+\|\nabla u\|_{\mathcal{H}^{m-1}}^{2}\right) d \tau+\int_{0}^{t}\|n \nabla \phi\|_{\mathcal{H}^{m}}^{2} d \tau\right\}
$$

where the pressure $p:=p_{1}+p_{2}$. Here, $p_{1}$ is the "Euler" part of the pressure which solves

$$
\left\{\begin{array}{l}
\Delta p_{1}=-\nabla \cdot(u \cdot \nabla u)-\nabla \cdot(n \nabla \phi) \quad \text { in } \quad \Omega \\
\partial_{\nu} p_{1}=-(u \cdot \nabla u) \cdot \nu-n \nabla \phi \cdot \nu \quad \text { on } \quad \partial \Omega
\end{array}\right.
$$

and $p_{2}^{\epsilon}$ is the "Navier-Stokes" part of the pressure which solves

$$
\left\{\begin{array}{l}
\Delta p_{2}=0 \quad \text { in } \quad \Omega \\
\partial_{\nu} p_{2}=\epsilon \Delta u \cdot \nu \text { on } \partial \Omega .
\end{array}\right.
$$

Note that here we use the convention that $\|\cdot\|_{\mathcal{H}^{m}}=0$ for $m<0$.

Proof. The estimate for $m=0$ has been given in Lemma 3.1 Now we assume that Lemma 3.2 holds for $|\alpha| \leq m-1$ and prove that it is still ture for $|\alpha|=m$. We apply $\mathcal{Z}^{\alpha}$ to (1.7) for $|\alpha|=m$ to obtain

$$
\mathcal{Z}^{\alpha} u_{t}+u \cdot \nabla \mathcal{Z}^{\alpha} u+\mathcal{Z}^{\alpha} \nabla p=\epsilon \mathcal{Z}^{\alpha} \Delta u-\mathcal{Z}^{\alpha}(n \nabla \phi)+\mathcal{C}_{1},
$$

where

$$
\mathcal{C}_{1}:=-\left[\mathcal{Z}^{\alpha}, u \cdot \nabla\right] u=-\sum_{|\beta| \geq 1, \beta+\gamma=\alpha} D_{\beta, \gamma} \mathcal{Z}^{\beta}(u) \cdot \mathcal{Z}^{\gamma} \nabla u-u \cdot\left[\mathcal{Z}^{\alpha}, \nabla\right] u .
$$

Multiplying (3.15) by $\mathcal{Z}^{\alpha} u$ and integrating by parts, we have

$$
\frac{1}{2} \frac{d}{d t}\left\|\mathcal{Z}^{\alpha} u\right\|^{2}+\left(\mathcal{Z}^{\alpha} \nabla p, \mathcal{Z}^{\alpha} u\right)=\epsilon\left(\mathcal{Z}^{\alpha} \Delta u, \mathcal{Z}^{\alpha} u\right)-\left(\mathcal{Z}^{\alpha}(n \nabla \phi), \mathcal{Z}^{\alpha} u\right)+\left(\mathcal{C}_{1}, \mathcal{Z}^{\alpha} u\right)
$$

First, we estimate the first term in the right-hand side of (3.16). We obtain

$$
\epsilon \int_{\Omega} \mathcal{Z}^{\alpha} \Delta u \cdot \mathcal{Z}^{\alpha} u d x=2 \epsilon \int_{\Omega}\left(\nabla \cdot \mathcal{Z}^{\alpha} S u\right) \cdot \mathcal{Z}^{\alpha} u d x+2 \epsilon \int_{\Omega}\left(\left[\mathcal{Z}^{\alpha}, \nabla \cdot\right] S u\right) \cdot \mathcal{Z}^{\alpha} u d x
$$

Now, by integrating by parts, we get from the first term in the right-hand side of (3.17) that

$$
\begin{aligned}
\epsilon \int_{\Omega}\left(\nabla \cdot \mathcal{Z}^{\alpha} S u\right) \cdot \mathcal{Z}^{\alpha} u d x= & -\epsilon \int_{\Omega} \mathcal{Z}^{\alpha} S u \cdot \nabla \mathcal{Z}^{\alpha} u d x+\epsilon \int_{\partial \Omega}\left(\left(\mathcal{Z}^{\alpha} S u\right) \cdot \nu\right) \cdot \mathcal{Z}^{\alpha} u d \sigma \\
= & -\epsilon\left\|S\left(\mathcal{Z}^{\alpha} u\right)\right\|^{2}-\epsilon \int_{\Omega}\left[\mathcal{Z}^{\alpha}, S\right] u \cdot \nabla \mathcal{Z}^{\alpha} u d x \\
& +\epsilon \int_{\partial \Omega}\left(\left(\mathcal{Z}^{\alpha} S u\right) \cdot \nu\right) \cdot \mathcal{Z}^{\alpha} u d \sigma
\end{aligned}
$$

Thanks to Lemma 2.1. there exists a $d_{0}>0$ such that

$$
\epsilon \int_{\Omega}\left(\nabla \cdot \mathcal{Z}^{\alpha} S u\right) \cdot \mathcal{Z}^{\alpha} u d x \leq-d_{0} \epsilon\left\|\nabla\left(\mathcal{Z}^{\alpha} u\right)\right\|^{2}+D_{m+2}\|u\|_{\mathcal{H}^{m}}^{2}
$$




$$
+D_{m+2} \epsilon\left\|\nabla \mathcal{Z}^{\alpha} u\right\|\|\nabla u\|_{\mathcal{H}^{m-1}}+\epsilon \int_{\partial \Omega}\left(\left(\mathcal{Z}^{\alpha} S u\right) \cdot \nu\right) \cdot \mathcal{Z}^{\alpha} u d \sigma .
$$

It remains to estimate the boundary term of (3.19). Before we treat the boundary term, we have the following observations. Due to the Navier boundary condition (1.24), we get

$$
\left|\Pi \partial_{\nu} \partial_{t}^{\alpha_{0}} u\right|_{H^{m}(\partial \Omega)} \leq\left|\theta\left(\partial_{t}^{\alpha_{0}} u\right)\right|_{H^{m}(\partial \Omega)}+2 \zeta\left|\Pi \partial_{t}^{\alpha_{0}} u\right|_{H^{m}(\partial \Omega)} \leq D\left|\partial_{t}^{\alpha_{0}} u\right|_{H^{m}(\partial \Omega)} .
$$

To estimate the normal part of $\partial_{\nu} u$, we can use the divergence free condition to write

$$
\nabla \cdot \partial_{t}^{\alpha_{0}} u=\partial_{\nu} \partial_{t}^{\alpha_{0}} u \cdot \nu+\left(\Pi \partial_{y_{1}} \partial_{t}^{\alpha_{0}} u\right)^{1}+\left(\Pi \partial_{y_{2}} \partial_{t}^{\alpha_{0}} u\right)^{2} .
$$

Hence, we easily get

$$
\left|\partial_{\nu} \partial_{t}^{\alpha_{0}} u \cdot \nu\right|_{H^{m-1}(\partial \Omega)} \leq D\left|\partial_{t}^{\alpha_{0}} u\right|_{H^{m}(\partial \Omega)} .
$$

From (3.20) and (3.22), we have

$$
\left|\nabla \partial_{t}^{\alpha_{0}} u\right|_{H^{m-1}(\partial \Omega)} \leq D\left|\partial_{t}^{\alpha_{0}} u\right|_{H^{m}(\partial \Omega)} .
$$

Thanks to $u \cdot n=0$ on the boundary, we immediately obtain that

$$
\left|\left(\mathcal{Z}^{\alpha} u\right) \cdot n\right|_{H^{1}(\partial \Omega)} \leq D\left|\partial_{t}^{\alpha_{0}} u\right|_{H^{m-\left|\alpha_{0}\right|(\partial \Omega)}}, \quad|\alpha|=m .
$$

Now we return to deal with the boundary term of (3.19) as follows

$$
\begin{aligned}
\epsilon \int_{\partial \Omega}\left(\left(\mathcal{Z}^{\alpha} S u\right) \cdot \nu\right) \cdot \mathcal{Z}^{\alpha} u d \sigma= & \epsilon \int_{\partial \Omega} \mathcal{Z}^{\alpha}(\Pi(S u \cdot \nu)) \cdot \Pi \mathcal{Z}^{\alpha} u d \sigma \\
& +\epsilon \int_{\partial \Omega} \mathcal{Z}^{\alpha}\left(\partial_{\nu} u \cdot \nu\right) \mathcal{Z}^{\alpha} u \cdot \nu d \sigma+\mathcal{C}_{b}
\end{aligned}
$$

where

$$
\begin{aligned}
\mathcal{C}_{b}:= & \epsilon \int_{\partial \Omega}\left[\mathcal{Z}^{\alpha}, \Pi\right](S u \cdot \nu) \cdot \Pi \mathcal{Z}^{\alpha} u d \sigma+\epsilon \int_{\partial \Omega}\left[\mathcal{Z}^{\alpha}, \nu\right]\left(S u^{\epsilon} \cdot \nu\right) \mathcal{Z}^{\alpha} u \cdot \nu d \sigma \\
& +\epsilon \sum_{\beta+\gamma=\alpha, \gamma \neq 0} D_{\beta, \gamma} \int_{\partial \Omega}\left(\mathcal{Z}^{\beta} S u \cdot \mathcal{Z}^{\gamma} \nu\right) \cdot \mathcal{Z}^{\alpha} u d \sigma .
\end{aligned}
$$

Thanks to the Navier boundary condition (1.11), we can easily get

$$
\left|\epsilon \int_{\partial \Omega} \mathcal{Z}^{\alpha}(\Pi(S u \cdot \nu)) \cdot \Pi \mathcal{Z}^{\alpha} u d \sigma\right| \leq \epsilon D_{m+2}\left|\partial_{t}^{\alpha_{0}} u\right|_{H^{m-\left|\alpha_{0}\right|}(\partial \Omega)}^{2} .
$$

We also note that $\mathcal{Z}^{\alpha} u \cdot n$ and $\mathcal{C}_{b}$ with $\alpha=\alpha_{0}$ vanish, so we assume $\alpha \neq \alpha_{0}$. From (3.23), we obtain that

$$
\begin{aligned}
\left|C_{b}\right| & \leq \epsilon D_{m+2}\left|\nabla \partial_{t}^{\alpha_{0}} u\right|_{H^{m-1-\left|\alpha_{0}\right|(\partial \Omega)}}\left|\partial_{t}^{\alpha_{0}} u\right|_{H^{m-\left|\alpha_{0}\right|(\partial \Omega)}} \\
& \leq \epsilon D_{m+2}\left|\partial_{t}^{\alpha_{0}} u\right|_{H^{m-\left|\alpha_{0}\right|(\partial \Omega)}}^{2} .
\end{aligned}
$$

By integrating by parts along the boundary, we have that

$$
\left|\epsilon \int_{\partial \Omega} \mathcal{Z}^{\alpha}\left(\partial_{\nu} u \cdot \nu\right) \mathcal{Z}^{\alpha} u \cdot \nu d \sigma\right| \leq \epsilon D\left|\partial_{\nu} \partial_{t}^{\alpha_{0}} u \cdot \nu\right|_{H^{m-1-\left|\alpha_{0}\right|(\partial \Omega)}}\left|\mathcal{Z}^{\alpha} u \cdot \nu\right|_{H^{1}(\partial \Omega)}
$$




$$
\leq \epsilon D\left|\partial_{t}^{\alpha_{0}} u\right|_{H^{m-\left|\alpha_{0}\right|(\partial \Omega)}}^{2} .
$$

Hence, we get from (3.19) and (3.25)-(3.27) that

$$
\begin{aligned}
\epsilon \int_{\Omega}\left(\nabla \cdot \mathcal{Z}^{\alpha} S u\right) \cdot \mathcal{Z}^{\alpha} u d \sigma \leq D_{m+2} & \left(\|u\|_{\mathcal{H}^{m}}^{2}+\epsilon\left\|\nabla \mathcal{Z}^{\alpha} u\right\|\|\nabla u\|_{\mathcal{H}^{m-1}}\right. \\
& \left.+\epsilon\left|\partial_{t}^{\alpha_{0}} u\right|_{H^{m-\left|\alpha_{0}\right|}(\partial \Omega)}^{2}\right)-d_{0} \epsilon\left\|\nabla\left(\mathcal{Z}^{\alpha} u\right)\right\|^{2}
\end{aligned}
$$

Next, we deal with the second term of the right-hand side of (3.17), i.e. $\epsilon \int_{\Omega}\left(\left[\mathcal{Z}^{\alpha}, \nabla \cdot\right] S u\right) \cdot \mathcal{Z}^{\alpha} u d x$. We can expand it as a sum of terms under the form

$$
\epsilon \int_{\Omega} \beta_{k} \partial_{k}\left(\mathcal{Z}^{\tilde{\alpha}} S u\right) \cdot \mathcal{Z}^{\alpha} u d x, \quad|\tilde{\alpha}| \leq m-1
$$

By using integrations by parts and (3.23), we have

$$
\begin{aligned}
\epsilon\left|\int_{\Omega} \beta_{k} \partial_{k}\left(\mathcal{Z}^{\tilde{\alpha}} S u\right) \cdot \mathcal{Z}^{\alpha} u d x\right| \leq D_{m+2} \epsilon & \left(\|\nabla u\|_{\mathcal{H}^{m}}\|\nabla u\|_{\mathcal{H}^{m-1}}+\|u\|_{\mathcal{H}^{m}}^{2}\right. \\
& \left.+\left|\partial_{t}^{\alpha_{0}} u\right|_{H^{m-\left|\alpha_{0}\right|(\partial \Omega)}}^{2}\right) .
\end{aligned}
$$

Consequently, from (3.28) and (3.29), we get

$$
\begin{aligned}
\epsilon\left|\int_{\Omega} \mathcal{Z}^{\alpha} \Delta u \cdot \mathcal{Z}^{\alpha} u d x\right| \leq D_{m+2} & \left\{\|u\|_{\mathcal{H}^{m}}^{2}+\epsilon\|\nabla u\|_{\mathcal{H}^{m}}\|\nabla u\|_{\mathcal{H}^{m-1}}\right. \\
& \left.+\epsilon\left|\partial_{t}^{\alpha_{0}} u\right|_{H^{m-\left|\alpha_{0}\right|}(\partial \Omega)}^{2}\right\}-d_{0} \epsilon\left\|\nabla\left(\mathcal{Z}^{\alpha} u\right)\right\|^{2} .
\end{aligned}
$$

Second, we estimate the term involving the pressure $p$ in (3.16). We note that $\left(\mathcal{Z}^{\alpha} \nabla p, \mathcal{Z}^{\alpha} u\right)$ with $\alpha=\alpha_{0}$ vanishes, so we deal with the case of $\alpha \neq \alpha_{0}$.

$$
\left|\int_{\Omega} \mathcal{Z}^{\alpha} \nabla p \cdot \mathcal{Z}^{\alpha} u d x\right| \leq\left\|\nabla^{2} p_{1}\right\|_{\mathcal{H}^{m-1}}\|u\|_{\mathcal{H}^{m}}+\left|\int_{\Omega} \mathcal{Z}^{\alpha} \nabla p_{2} \cdot \mathcal{Z}^{\alpha} u d x\right| .
$$

Now, we focus on the last term in (3.31). By integrating by parts, we obtain that

$$
\begin{aligned}
\left|\int_{\Omega} \mathcal{Z}^{\alpha} \nabla p_{2} \cdot \mathcal{Z}^{\alpha} u d x\right| \leq D_{m+2} & \left(\left\|\nabla p_{2}\right\|_{\mathcal{H}^{m-1}}\|u\|_{\mathcal{H}^{m}}+\left\|\nabla p_{2}\right\|_{\mathcal{H}^{m-1}}\left\|\nabla \mathcal{Z}^{\alpha} u\right\|\right. \\
+ & \left.\left|\int_{\partial \Omega} \mathcal{Z}^{\alpha} p_{2} \mathcal{Z}^{\alpha} u \cdot \nu d \sigma\right|\right) .
\end{aligned}
$$

By integrating by parts along the boundary and Lemma 2.3, we get

$$
\begin{aligned}
& \left|\int_{\partial \Omega} \mathcal{Z}^{\alpha} p_{2} \mathcal{Z}^{\alpha} u \cdot \nu d \sigma\right| \\
\leq & D_{m+2}\left|\mathcal{Z}^{\widetilde{\alpha}} p_{2}\right|_{L^{2}(\partial \Omega)}\left|\mathcal{Z}^{\alpha} u \cdot \nu\right|_{H^{1}(\partial \Omega)} \\
\leq & D_{m+2}\left(\left\|\nabla p_{2}\right\|_{\mathcal{H}^{m-1}}+\left\|p_{2}\right\|_{\mathcal{H}^{m-1}}\right)\left(\|\nabla u\|_{\mathcal{H}^{m}}+\|u\|_{\mathcal{H}^{m}}\right),
\end{aligned}
$$

where $|\widetilde{\alpha}|=m-1$. From (3.31)-(3.33), we get

$$
\begin{aligned}
\left|\int_{\Omega} \mathcal{Z}^{\alpha} \nabla p \cdot \mathcal{Z}^{\alpha} u d x\right| \leq & D_{m+2}\left\|\nabla^{2} p_{1}\right\|_{\mathcal{H}^{m-1}}\|u\|_{\mathcal{H}^{m}}+D_{m+2}\left(\left\|\nabla p_{2}\right\|_{\mathcal{H}^{m-1}}\right. \\
& \left.+\left\|p_{2}\right\|_{\mathcal{H}^{m-1}}\right)\left(\|\nabla u\|_{\mathcal{H}^{m}}+\|u\|_{\mathcal{H}^{m}}\right) .
\end{aligned}
$$


Finally, we estimate the commutator term. By using Lemma 2.2 we have

$$
\begin{aligned}
\int_{0}^{t}\left\|\mathcal{C}_{1}\right\|^{2} d \tau \leq & \sum_{|\beta| \geq 1, \beta+\gamma=\alpha} D_{\beta, \gamma} \int_{0}^{t}\left\|\mathcal{Z}^{\beta}(u) \cdot \mathcal{Z}^{\gamma} \nabla u\right\|^{2} d \tau+\int_{0}^{t}\left\|u \cdot\left[\mathcal{Z}^{\alpha}, \nabla\right] u\right\|^{2} d \tau \\
\leq D & \left\{\|\mathcal{Z} u\|_{L_{x, t}^{\infty}}^{2} \int_{0}^{t}\|\nabla u\|_{\mathcal{H}^{m-1}}^{2} d \tau+\|\nabla u\|_{L_{x, t}^{\infty}}^{2} \int_{0}^{t}\|\mathcal{Z} u\|_{\mathcal{H}^{m-1}}^{2} d \tau\right. \\
& \left.\quad+\|u\|_{L_{x, t}^{\infty}}^{2} \int_{0}^{t}\|\nabla u\|_{\mathcal{H}^{m-1}}^{2} d \tau\right\} \\
\leq & D_{m+1} M(t) \int_{0}^{t}\left(\|\nabla u\|_{\mathcal{H}^{m-1}}^{2}+\|u\|_{\mathcal{H}^{m}}^{2}\right) d \tau
\end{aligned}
$$

Consequently, from (3.30), (3.34)-(3.35), Lemma 2.3, Young's inequality and the assumptions with respect to $|\alpha| \leq m-1$, we get (3.12). This ends the proof of Lemma 3.2

Next, we give the higher order estimate of $(n, c)$.

Lemma 3.5. For every $m \geq 0$, a smooth solution of the problem (1.5)-(1.11) satisfies the estimate

$$
\begin{aligned}
\sup _{0 \leq \tau \leq t}\left(\|(n, c)\|_{\mathcal{H}^{m}}^{2}\right)+d \int_{0}^{t}\|\nabla(n, c)\|_{\mathcal{H}^{m}}^{2} d \tau \\
\leq D_{m+2}\left\{\|\left(n_{0}, c_{0} \|_{\mathcal{H}^{m}}^{2}+(1+P(M(t))) \int_{0}^{t}\left(\|(n, c, u)\|_{\mathcal{H}^{m}}^{2}+\|\nabla c\|_{\mathcal{H}^{m}}^{2}\right.\right.\right. \\
\left.\left.+\|\nabla n\|_{\mathcal{H}^{m-1}}^{2}+\left\|\nabla^{2} c\right\|_{\mathcal{H}^{m-1}}^{2}\right) d \tau+\delta \int_{0}^{t}\left\|\nabla^{2} n\right\|_{\mathcal{H}^{m-1}}^{2} d \tau\right\},
\end{aligned}
$$

where $\delta>0$ is a small enough constant.

Proof. The estimate for $m=0$ has been given in Lemmas 3.2 and 3.3 Now we assume that Lemma 3.5 holds for $|\alpha| \leq m-1$ and prove that it is still true for $|\alpha|=m$. We apply $\mathcal{Z}^{\alpha}$ to (1.5) for $|\alpha|=m$ to obtain that

$$
\mathcal{Z}^{\alpha} n_{t}+u \cdot \nabla \mathcal{Z}^{\alpha} n=\mathcal{Z}^{\alpha} \Delta n-\mathcal{Z}^{\alpha}(\nabla \cdot(n \nabla c))+\mathcal{C}_{2}
$$

where

$$
\mathcal{C}_{2}:=-\left[\mathcal{Z}^{\alpha}, u \cdot \nabla\right] n=-\sum_{|\beta| \geq 1, \beta+\gamma=\alpha} D_{\beta, \gamma} \mathcal{Z}^{\beta}(u) \cdot \mathcal{Z}^{\gamma} \nabla n-u \cdot\left[\mathcal{Z}^{\alpha}, \nabla\right] n .
$$

Multiplying (3.37) by $\mathcal{Z}^{\alpha} n$ and integrating by parts, we have

$$
\frac{1}{2} \frac{d}{d t}\left\|\mathcal{Z}^{\alpha} n\right\|^{2}=\left(\mathcal{Z}^{\alpha} \Delta n, \mathcal{Z}^{\alpha} n\right)-\left(\mathcal{Z}^{\alpha}(\nabla \cdot(n \nabla c)), \mathcal{Z}^{\alpha} n\right)+\left(\mathcal{C}_{2}, \mathcal{Z}^{\alpha} n\right) .
$$

First, we estimate the first term in the right-hand side of (3.38). By integrating by parts, we get

$$
\int_{\Omega} \mathcal{Z}^{\alpha} \Delta n \mathcal{Z}^{\alpha} n d x=\int_{\Omega} \nabla \cdot\left(\mathcal{Z}^{\alpha} \nabla n\right) \mathcal{Z}^{\alpha} n d x+\int_{\Omega}\left[\mathcal{Z}^{\alpha}, \nabla \cdot\right] \nabla n \mathcal{Z}^{\alpha} n d x
$$




$$
\begin{aligned}
= & -\int_{\Omega} \mathcal{Z}^{\alpha} \nabla n \nabla \mathcal{Z}^{\alpha} n d x+\int_{\Omega}\left[\mathcal{Z}^{\alpha}, \nabla \cdot\right] \nabla n \mathcal{Z}^{\alpha} n d x \\
& +\int_{\partial \Omega} \nu \cdot \mathcal{Z}^{\alpha} \nabla n \mathcal{Z}^{\alpha} n d \sigma .
\end{aligned}
$$

Thanks to Lemma 2.3, there exists a constant $d>0$ such that

$$
\int_{\Omega} \mathcal{Z}^{\alpha} \Delta n \mathcal{Z}^{\alpha} n d x \leq-d\|\nabla n\|_{\mathcal{H}^{m}}^{2}+D_{\delta} D_{m+2}\left(\|n\|_{\mathcal{H}^{m}}^{2}+\|\nabla n\|_{\mathcal{H}^{m-1}}^{2}\right)+\delta\left\|\nabla^{2} n\right\|_{\mathcal{H}^{m-1}}^{2}
$$

Next, we deal with the second term in the right-hand side of (3.38). By integrating by parts, we get

$$
\begin{aligned}
-\int_{\Omega} \mathcal{Z}^{\alpha}(\nabla \cdot(n \nabla c)) \mathcal{Z}^{\alpha} n d x= & \int_{\Omega} \mathcal{Z}^{\alpha}(n \nabla c) \nabla \mathcal{Z}^{\alpha} n d x-\int_{\Omega}\left[\mathcal{Z}^{\alpha}, \nabla \cdot\right](n \nabla c) \mathcal{Z}^{\alpha} n d x \\
& -\int_{\partial \Omega} \nu \cdot \mathcal{Z}^{\alpha}(n \nabla c) \mathcal{Z}^{\alpha} n d \sigma
\end{aligned}
$$

For the last term of (3.41), by using Lemmas 2.2 and 2.3, and (1.10), we obtain that

$$
\begin{aligned}
\left|\int_{0}^{t} \int_{\partial \Omega} \nu \cdot \mathcal{Z}^{\alpha}(n \nabla c) \mathcal{Z}^{\alpha} n d \sigma d \tau\right| \leq & D_{\delta} D_{m+2}(1+M(t)) \int_{0}^{t}\left\{\|n\|_{\mathcal{H}^{m}}^{2}+\|\nabla(n, c)\|_{\mathcal{H}^{m-1}}^{2}\right. \\
& \left.+\left\|\nabla^{2} c\right\|_{\mathcal{H}^{m-1}}^{2}\right\} d \tau+\delta \int_{0}^{t}\|\nabla n\|_{\mathcal{H}^{m}}^{2} d \tau . \quad(3.42)
\end{aligned}
$$

For the commutator term in (3.41), we can expand it as a sum of terms under the form

$$
\int_{\Omega} \beta_{k} \partial_{k} \mathcal{Z}^{\tilde{\alpha}}(n \nabla c) \mathcal{Z}^{\alpha} n d x, \quad|\tilde{\alpha}| \leq m-1 .
$$

By using Lemma 2.2, we easily get that

$$
\begin{aligned}
\left|\int_{0}^{t} \int_{\Omega}\left[\mathcal{Z}^{\alpha}, \nabla \cdot\right](n \nabla c) \mathcal{Z}^{\alpha} n d x d \tau\right| \leq D_{m+1} M(t) \int_{0}^{t} & \left(\|n\|_{\mathcal{H}^{m}}^{2}+\|\nabla(n, c)\|_{\mathcal{H}^{m-1}}^{2}\right. \\
& \left.+\left\|\nabla^{2} c\right\|_{\mathcal{H}^{m-1}}^{2}\right) d \tau
\end{aligned}
$$

Also, by using Lemma 2.2, we obtain that

$$
\begin{aligned}
\left|\int_{0}^{t} \int_{\Omega} \mathcal{Z}^{\alpha}(n \nabla c) \nabla \mathcal{Z}^{\alpha} n d x d \tau\right| \leq & D_{\delta} D_{m+2} M(t) \int_{0}^{t}\left(\|n\|_{\mathcal{H}^{m}}^{2}+\|\nabla c\|_{\mathcal{H}^{m}}^{2}\right) d \tau \\
& +\delta \int_{0}^{t}\|\nabla n\|_{\mathcal{H}^{m}}^{2} d \tau
\end{aligned}
$$

Therefore, from (3.42)-(3.44), we get that

$$
\begin{aligned}
& \quad\left|\int_{0}^{t} \int_{\Omega} \mathcal{Z}^{\alpha}(\nabla \cdot(n \nabla c)) \mathcal{Z}^{\alpha} n d x d \tau\right| \\
& \leq D_{\delta} D_{m+2}(1+M(t)) \int_{0}^{t}\left(\|n\|_{\mathcal{H}^{m}}^{2}+\|\nabla n\|_{\mathcal{H}^{m-1}}^{2}\right. \\
& \left.\quad+\|\nabla c\|_{\mathcal{H}^{m}}^{2}+\left\|\nabla^{2} c\right\|_{\mathcal{H}^{m-1}}^{2}\right) d \tau+\delta \int_{0}^{t}\|\nabla n\|_{\mathcal{H}^{m}}^{2} d \tau .
\end{aligned}
$$


Finally, we estimate the commutator term in (3.38). Similar to (3.35), by using Lemma 2.2 we have

$$
\left|\int_{0}^{t} \int_{\Omega} \mathcal{C}_{2} \mathcal{Z}^{\alpha} n d x d \tau\right| \leq D_{m+1} M(t) \int_{0}^{t}\left(\|(n, u)\|_{\mathcal{H}^{m}}^{2}+\|\nabla n\|_{\mathcal{H}^{m-1}}^{2}\right) d \tau .
$$

Similar to $n$, we apply $\mathcal{Z}^{\alpha}$ to (1.6) for $|\alpha|=m$ to obtain

$$
\left.\mathcal{Z}^{\alpha} c_{t}+u \cdot \nabla \mathcal{Z}^{\alpha} c=\mathcal{Z}^{\alpha} \Delta c-\mathcal{Z}^{\alpha}(n c)\right)+\mathcal{C}_{3},
$$

where

$$
\mathcal{C}_{3}:=-\left[\mathcal{Z}^{\alpha}, u \cdot \nabla\right] c .
$$

Multiplying (3.47) by $\mathcal{Z}^{\alpha} c$ and integrating by parts, we have

$$
\frac{1}{2} \frac{d}{d t}\left\|\mathcal{Z}^{\alpha} c\right\|^{2}=\left(\mathcal{Z}^{\alpha} \Delta c, \mathcal{Z}^{\alpha} c\right)-\left(\mathcal{Z}^{\alpha}(n c), \mathcal{Z}^{\alpha} c\right)+\left(\mathcal{C}_{3}, \mathcal{Z}^{\alpha} c\right) .
$$

By using Lemma 2.2, we can easily obtain

$$
\left|\int_{0}^{t} \int_{\Omega} \mathcal{Z}^{\alpha}(n c) \mathcal{Z}^{\alpha} c d x d \tau\right| \leq D_{m+2} M(t) \int_{0}^{t}\left(\|n\|_{\mathcal{H}^{m}}^{2}+\|c\|_{\mathcal{H}^{m}}^{2}\right) d \tau .
$$

Similar to (3.40) and (3.46), we can directly get

$$
\begin{aligned}
& \int_{\Omega} \mathcal{Z}^{\alpha} \Delta c \mathcal{Z}^{\alpha} c d x \leq-d\|\nabla c\|_{\mathcal{H}^{m}}^{2}+D_{\delta} D_{m+2}\left(\|c\|_{\mathcal{H}^{m}}^{2}+\|\nabla c\|_{\mathcal{H}^{m-1}}^{2}\right)+\delta\left\|\nabla^{2} c\right\|_{\mathcal{H}^{m-1}}^{2} \\
& \left|\int_{0}^{t} \int_{\Omega} \mathcal{C}_{3} \mathcal{Z}^{\alpha} c d x d \tau\right| \leq D_{m+1} M(t) \int_{0}^{t}\left(\|(c, u)\|_{\mathcal{H}^{m}}^{2}+\|\nabla c\|_{\mathcal{H}^{m-1}}^{2}\right) d \tau
\end{aligned}
$$

Consequently, from (3.40), (3.45)-(3.46), (3.49)-(3.51), and the assumptions with respect to $|\alpha| \leq m-1$, we get (3.36). This ends the proof of Lemma 3.5.

3.2. Conormal Energy Estimates for $\nabla n$ and $\nabla c$. In this subsection, we shall give some uniform estimates to $\|\nabla n\|_{\mathcal{H}^{m-1}}$ and $\|\nabla c\|_{\mathcal{H}^{m}}$. First, we deal with $\nabla n$. We have

Lemma 3.6. For every $m \geq 1$, a smooth solution of the problem (1.5)-(1.11) satisfies the estimate

$$
\begin{aligned}
& \sup _{0 \leq \tau \leq t}\|\nabla n\|_{\mathcal{H}^{m-1}}^{2}+\int_{0}^{t}\|\Delta n\|_{\mathcal{H}^{m-1}}^{2} d \tau \\
& \leq D_{m+2}\left\{\left\|\nabla n_{0}\right\|_{\mathcal{H}^{m-1}}^{2}+\delta \int_{0}^{t}\|\nabla \Delta n\|_{\mathcal{H}^{m-2}}^{2} d \tau+\delta \int_{0}^{t}\|\nabla \Delta c\|_{\mathcal{H}^{m-1}}^{2} d \tau\right. \\
& +D_{\delta}(1+P(M(t))) \int_{0}^{t}\left(\|(n, u)\|_{\mathcal{H}^{m-1}}^{2}+\|\nabla(n, c, u)\|_{\mathcal{H}^{m-1}}^{2}\right. \\
& \left.\left.+\left\|\nabla^{2}(n, c)\right\|_{\mathcal{H}^{m-1}}^{2}\right) d \tau\right\}
\end{aligned}
$$

where $\delta>0$ is a small enough constant. 
Proof. Multiplying (1.5) by $\Delta n$ yields that

$$
\int_{\Omega} n_{t} \Delta n d x+\int_{\Omega} u \cdot \nabla n \Delta n d x=\int_{\Omega} \Delta n \Delta n d x-\int_{\Omega} \nabla \cdot(n \nabla c) \Delta n d x .
$$

Integrating by parts and using the boundary condition (1.10), we obtain that

$$
\int_{\Omega} n_{t} \Delta n d x=-\frac{1}{2} \frac{d}{d t} \int_{\Omega}|\nabla n|^{2} d x
$$

Furthermore, by using Young's inequality, we get

$$
\sup _{0 \leq \tau \leq t}\|\nabla n\|^{2}+\int_{0}^{t}\|\Delta n\|^{2} d \tau \leq D\left\{\left\|\nabla n_{0}\right\|^{2}+M(t) \int_{0}^{t}\left(\|\nabla n\|^{2}+\|n\|^{2}\right) d \tau\right\} .
$$

Hence, the case of $|\alpha|=1$ is true.

Next, we consider the higher order estimates. Assume that (3.52) has been proved for $|\alpha| \leq m-2$, we need to prove it holds for $|\alpha|=m-1$. By applying $\mathcal{Z}^{\alpha} \nabla$ with $|\alpha|=m-1$ to (1.5), we obtain that

$$
\mathcal{Z}^{\alpha} \nabla n_{t}-\mathcal{Z}^{\alpha} \nabla \Delta n=-\mathcal{Z}^{\alpha} \nabla(\nabla n \cdot \nabla c)-\mathcal{Z}^{\alpha} \nabla(n \Delta c)-\mathcal{Z}^{\alpha} \nabla(u \cdot \nabla n) .
$$

Multiplying (3.55) by $\mathcal{Z}^{\alpha} \nabla n$ leads to

$$
\begin{aligned}
& \frac{1}{2} \frac{d}{d t} \int_{\Omega}\left|\mathcal{Z}^{\alpha} \nabla n\right|^{2} d x-\int_{\Omega} \mathcal{Z}^{\alpha} \nabla \Delta n \mathcal{Z}^{\alpha} \nabla n d x \\
= & -\int_{\Omega} \mathcal{Z}^{\alpha} \nabla(\nabla n \cdot \nabla c) \mathcal{Z}^{\alpha} \nabla n d x-\int_{\Omega} \mathcal{Z}^{\alpha} \nabla(n \Delta c) \mathcal{Z}^{\alpha} \nabla n d x \\
& -\int_{\Omega} \mathcal{Z}^{\alpha} \nabla(u \cdot \nabla n) \mathcal{Z}^{\alpha} \nabla n d x .
\end{aligned}
$$

By using integration by parts and the boundary condition (1.10), we obtain that

$$
\begin{aligned}
& -\int_{\Omega} \mathcal{Z}^{\alpha} \nabla \Delta n \mathcal{Z}^{\alpha} \nabla n d x \\
= & -\int_{\partial \Omega} \mathcal{Z}^{\alpha} \Delta n \mathcal{Z}^{\alpha} \nabla n \cdot \nu d \sigma+\int_{\Omega}\left|\operatorname{div}\left(\mathcal{Z}^{\alpha} \nabla n\right)\right|^{2} d x \\
& +\int_{\Omega}\left[\mathcal{Z}^{\alpha}, \operatorname{div}\right] \nabla n \operatorname{div}\left(\mathcal{Z}^{\alpha} \nabla n\right) d x-\int_{\Omega}\left[\mathcal{Z}^{\alpha}, \nabla\right] \Delta n \cdot \mathcal{Z}^{\alpha} \nabla n d x .
\end{aligned}
$$

Hence, we have

$$
\begin{aligned}
& \frac{1}{2} \frac{d}{d t} \int_{\Omega}\left|\mathcal{Z}^{\alpha} \nabla n\right|^{2} d x+\int_{\Omega}\left|\operatorname{div}\left(\mathcal{Z}^{\alpha} \nabla n\right)\right|^{2} d x \\
= & -\int_{\Omega} \mathcal{Z}^{\alpha} \nabla(\nabla n \cdot \nabla c) \mathcal{Z}^{\alpha} \nabla n d x-\int_{\Omega} \mathcal{Z}^{\alpha} \nabla(n \Delta c) \mathcal{Z}^{\alpha} \nabla n d x \\
& -\int_{\Omega} \mathcal{Z}^{\alpha} \nabla(u \cdot \nabla n) \mathcal{Z}^{\alpha} \nabla n d x+\int_{\partial \Omega} \mathcal{Z}^{\alpha} \Delta n \mathcal{Z}^{\alpha} \nabla n \cdot \nu d \sigma \\
& -\int_{\Omega}\left[\mathcal{Z}^{\alpha}, \operatorname{div}\right] \nabla n \operatorname{div}\left(\mathcal{Z}^{\alpha} \nabla n\right) d x+\int_{\Omega}\left[\mathcal{Z}^{\alpha}, \nabla\right] \Delta n \cdot \mathcal{Z}^{\alpha} \nabla n d x .
\end{aligned}
$$

First, applying Young's inequality, we can easily arrive at

$$
\left|\int_{0}^{t} \int_{\Omega}\left[\mathcal{Z}^{\alpha}, \operatorname{div}\right] \nabla n \operatorname{div}\left(\mathcal{Z}^{\alpha} \nabla n\right) d x d \tau-\int_{0}^{t} \int_{\Omega}\left[\mathcal{Z}^{\alpha}, \nabla\right] \Delta n \cdot \mathcal{Z}^{\alpha} \nabla n d x d \tau\right|
$$




$$
\begin{aligned}
\leq & \delta \int_{0}^{t}\|\nabla \Delta n\|_{\mathcal{H}^{m-2}}^{2} d \tau+\delta \int_{0}^{t}\left\|\operatorname{div}\left(\mathcal{Z}^{\alpha} \nabla n\right)\right\|^{2} d \tau \\
& +D_{\delta} D_{m+1}\left(\int_{0}^{t}\left\|\nabla^{2} n\right\|_{\mathcal{H}^{m-2}}^{2} d \tau+\int_{0}^{t}\|\nabla n\|_{\mathcal{H}^{m-1}}^{2} d \tau\right)
\end{aligned}
$$

Next, using Lemma 2.2, we have

$$
\begin{aligned}
& \left|\int_{0}^{t} \int_{\Omega} \mathcal{Z}^{\alpha} \nabla(\nabla n \cdot \nabla c) \mathcal{Z}^{\alpha} \nabla n d x d \tau\right| \\
\leq & D_{m+2} P(M(t)) \int_{0}^{t}\left(\|\nabla n\|_{\mathcal{H}^{m-1}}^{2}+\|\nabla c\|_{\mathcal{H}^{m-1}}^{2}+\left\|\nabla^{2} n\right\|_{\mathcal{H}^{m-1}}^{2}+\left\|\nabla^{2} c\right\|_{\mathcal{H}^{m-1}}^{2}\right) d \tau .
\end{aligned}
$$

Furthermore, based on Lemma 2.2 and Young's inequality, we obtain

$$
\begin{aligned}
& \quad\left|\int_{0}^{t} \int_{\Omega} \mathcal{Z}^{\alpha} \nabla(n \Delta c) \mathcal{Z}^{\alpha} \nabla n d x d \tau\right| \\
& \leq \delta \int_{0}^{t}\|\nabla \Delta c\|_{\mathcal{H}^{m-1}}^{2} d \tau+D_{\delta} D_{m+2} P(M(t)) \\
& \quad \times \int_{0}^{t}\left(\|\nabla n\|_{\mathcal{H}^{m-1}}^{2}+\|\Delta c\|_{\mathcal{H}^{m-1}}^{2}+\|n\|_{\mathcal{H}^{m-1}}^{2}\right) d \tau .
\end{aligned}
$$

Now, we turn to estimate the boundary term in (3.56). Note that when $\left|\alpha_{0}\right|=$ $m-1$ or $\left|\alpha_{13}\right| \neq 0$, this term vanishes. So we can integrate by parts along the boundary to deduce that

$$
\left|\int_{0}^{t} \int_{\partial \Omega} \mathcal{Z}^{\alpha} \Delta n \mathcal{Z}^{\alpha} \nabla n \cdot \nu d \sigma d \tau\right| \leq \int_{0}^{t}\left|\partial_{t}^{\alpha_{0}} Z_{y}^{\beta} \Delta n\right|_{L^{2}(\partial \Omega)}\left|\mathcal{Z}^{\alpha} \nabla n \cdot \nu\right|_{H^{1}(\partial \Omega)} d \tau
$$

where $|\beta|=m-\left|\alpha_{0}\right|-1$. Due to Lemma 2.3, we arrive at

$$
\left|\partial_{t}^{\alpha_{0}} Z_{y}^{\beta} \Delta n\right|_{L^{2}(\partial \Omega)} \leq D_{m+2}\left(\|\nabla \Delta n\|_{\mathcal{H}^{m-2}}^{\frac{1}{2}}+\|\Delta n\|_{\mathcal{H}^{m-2}}^{\frac{1}{2}}\right)\|\Delta n\|_{\mathcal{H}^{m-2}}^{\frac{1}{2}} .
$$

With the help of the boundary condition (1.10) and Lemma 2.3, we have

$$
\left|\mathcal{Z}^{\alpha} \nabla n \cdot \nu\right|_{H^{1}(\partial \Omega)} \leq D_{m+2}\left(\left\|\nabla^{2} n\right\|_{\mathcal{H}^{m-1}}^{\frac{1}{2}}+\|\nabla n\|_{\mathcal{H}^{m-1}}^{\frac{1}{2}}\right)\|\nabla n\|_{\mathcal{H}^{m-1}}^{\frac{1}{2}}
$$

Based on (3.60)-(3.62) and Young's inequality, we can get

$$
\begin{aligned}
\left|\int_{0}^{t} \int_{\partial \Omega} \mathcal{Z}^{\alpha} \Delta n \mathcal{Z}^{\alpha} \nabla n \cdot \nu d \sigma d \tau\right| \leq & \delta \int_{0}^{t}\|\nabla \Delta n\|_{\mathcal{H}^{m-2}}^{2} d \tau+D_{\delta} D_{m+2} \int_{0}^{t}\left(\|\nabla n\|_{\mathcal{H}^{m-1}}^{2}\right. \\
& \left.+\left\|\nabla^{2} n\right\|_{\mathcal{H}^{m-1}}^{2}\right) d \tau
\end{aligned}
$$

Finally, we deal with the term involving $u$ in (3.56). Integrating by parts leads to that

$$
\begin{aligned}
& \int_{\Omega} \mathcal{Z}^{\alpha} \nabla(u \cdot \nabla n) \mathcal{Z}^{\alpha} \nabla n d x \\
= & \int_{\partial \Omega} \mathcal{Z}^{\alpha}(u \cdot \nabla n) \mathcal{Z}^{\alpha} \nabla n \cdot \nu d x+\int_{\Omega} \mathcal{Z}^{\alpha}(u \cdot \nabla n) \operatorname{div}\left(\mathcal{Z}^{\alpha} \nabla n\right) d x
\end{aligned}
$$




$$
+\int_{\Omega}\left[\mathcal{Z}^{\alpha}, \nabla\right](u \cdot \nabla n) \mathcal{Z}^{\alpha} \nabla n d x .
$$

By using Lemmas 2.2 and 2.3, we get

$$
\begin{aligned}
& \left|\int_{0}^{t} \int_{\partial \Omega} \mathcal{Z}^{\alpha}(u \cdot \nabla n) \mathcal{Z}^{\alpha} \nabla n \cdot \nu d x d \tau\right| \\
& \leq D_{m+2}(1+P(M(t))) \int_{0}^{t}\left(\left\|\nabla^{2} n\right\|_{\mathcal{H}^{m-1}}^{2}+\|\nabla n\|_{\mathcal{H}^{m-1}}^{2}+\|u\|_{\mathcal{H}^{m-1}}^{2}+\|\nabla u\|_{\mathcal{H}^{m-1}}^{2}\right) d \tau .
\end{aligned}
$$

Applying Young's inequality and Lemma 2.2, we obtain that

$$
\begin{aligned}
& \quad\left|\int_{0}^{t} \int_{\Omega} \mathcal{Z}^{\alpha}(u \cdot \nabla n) \operatorname{div}\left(\mathcal{Z}^{\alpha} \nabla n\right) d x d \tau+\int_{0}^{t} \int_{\Omega}\left[\mathcal{Z}^{\alpha}, \nabla\right](u \cdot \nabla n) \mathcal{Z}^{\alpha} \nabla n d x d \tau\right| \\
& \leq \delta \int_{0}^{t}\left\|\operatorname{div}\left(\mathcal{Z}^{\alpha} \nabla n\right)\right\|^{2} d \tau+D_{\delta} D_{m+2} P(M(t)) \int_{0}^{t}\left(\|\nabla n\|_{\mathcal{H}^{m-1}}^{2}+\left\|\nabla^{2} n\right\|_{\mathcal{H}^{m-2}}^{2}\right. \\
& \left.\quad+\|u\|_{\mathcal{H}^{m-2}}^{2}+\|\nabla u\|_{\mathcal{H}^{m-2}}^{2}\right) d \tau .
\end{aligned}
$$

Combination of (3.64)-(3.66) yields that

$$
\begin{aligned}
& \left|\int_{0}^{t} \int_{\Omega} \mathcal{Z}^{\alpha} \nabla(u \cdot \nabla n) \mathcal{Z}^{\alpha} \nabla n d x d \tau\right| \\
\leq & \delta \int_{0}^{t}\left\|\operatorname{div}\left(\mathcal{Z}^{\alpha} \nabla n\right)\right\|^{2} d \tau+D_{m+2}(1+P(M(t))) \\
& \times \int_{0}^{t}\left(\left\|\nabla^{2} n\right\|_{\mathcal{H}^{m-1}}^{2}+\|\nabla n\|_{\mathcal{H}^{m-1}}^{2}+\|u\|_{\mathcal{H}^{m-1}}^{2}+\|\nabla u\|_{\mathcal{H}^{m-1}}^{2}\right) d \tau .
\end{aligned}
$$

Based on the elliptic regularity results with Neumann boundary condition, we obtain that

$$
\left\|\nabla^{2} n\right\|_{\mathcal{H}^{m-1}}^{2} \leq D_{m+2}\left(\|\nabla n\|_{\mathcal{H}^{m-1}}^{2}+\|\Delta n\|_{\mathcal{H}^{m-1}}^{2}\right)
$$

Consequently, the combination of (3.56)-(3.58), (3.63), (3.67)-(3.68) and the inductive assumption yield (3.52). Therefore, we complete the proof of Lemma 3.6 .

Next, we give the uniform estimate to $\nabla c$.

Lemma 3.7. For every $m \geq 0$, a smooth solution of the problem (1.5)-(1.11) satisfies the estimate

$$
\begin{aligned}
& \sup _{0 \leq \tau \leq t}\|\nabla c\|_{\mathcal{H}^{m}}^{2}+\int_{0}^{t}\|\Delta c\|_{\mathcal{H}^{m}}^{2} d \tau \\
& \leq D_{m+2}\left\{\left\|\nabla c_{0}\right\|_{\mathcal{H}^{m}}^{2}+\delta \int_{0}^{t}\|\nabla \Delta c\|_{\mathcal{H}^{m-1}}^{2} d \tau+\delta \int_{0}^{t}\|\nabla n\|_{\mathcal{H}^{m}}^{2} d \tau\right. \\
&+D_{\delta}(1+P(M(t))) \int_{0}^{t}\left(\|(n, c, u)\|_{\mathcal{H}^{m}}^{2}+\|\nabla c\|_{\mathcal{H}^{m}}^{2}+\|\nabla u\|_{\mathcal{H}^{m-1}}^{2}\right. \\
&\left.\left.+\left\|\nabla^{2} c\right\|_{\mathcal{H}^{m-1}}^{2}\right) d \tau\right\},
\end{aligned}
$$


where $\delta>0$ is a small enough constant.

Proof. Multiplying (1.6) by $\Delta n$ yields that

$$
\int_{\Omega} c_{t} \Delta c d x+\int_{\Omega} u \cdot \nabla c \Delta c d x=\int_{\Omega} \Delta c \Delta c d x-\int_{\Omega} n c \Delta c d x .
$$

Integration by parts and the boundary condition (1.10) lead to

$$
\int_{\Omega} c_{t} \Delta c d x=-\frac{1}{2} \frac{d}{d t} \int_{\Omega}|\nabla c|^{2} d x
$$

By using Young's inequality, we arrive at

$$
\sup _{0 \leq \tau \leq t}\|\nabla c\|^{2}+\int_{0}^{t}\|\Delta c\|^{2} d \tau \leq D\left\{\left\|\nabla c_{0}\right\|^{2}+M(t) \int_{0}^{t}\left(\|\nabla c\|^{2}+\|c\|^{2}\right) d \tau\right\} .
$$

Hence, (3.69) holds in the case of $|\alpha|=0$.

Next, we deal with the higher order estimates. Assume that (3.69) has been proved for $|\alpha| \leq m-1$, we need to show it still holds for $|\alpha|=m$. By applying $\mathcal{Z}^{\alpha} \nabla$ with $|\alpha|=m$ to (1.6), we obtain that

$$
\mathcal{Z}^{\alpha} \nabla c_{t}-\mathcal{Z}^{\alpha} \nabla \Delta n=-\mathcal{Z}^{\alpha} \nabla(n c)-\mathcal{Z}^{\alpha} \nabla(u \cdot \nabla c) .
$$

By multiplying (3.73) by $\mathcal{Z}^{\alpha} \nabla c$, we obtain that

$$
\begin{aligned}
\frac{1}{2} \frac{d}{d t} \int_{\Omega}\left|\mathcal{Z}^{\alpha} \nabla c\right|^{2} d x-\int_{\Omega} \mathcal{Z}^{\alpha} \nabla \Delta c \mathcal{Z}^{\alpha} \nabla c d x & =-\int_{\Omega} \mathcal{Z}^{\alpha} \nabla(n c) \mathcal{Z}^{\alpha} \nabla c d x \\
& -\int_{\Omega} \mathcal{Z}^{\alpha} \nabla(u \cdot \nabla c) \mathcal{Z}^{\alpha} \nabla c d x .
\end{aligned}
$$

By integrating by parts and using the boundary condition (1.10), we obtain that

$$
\begin{aligned}
& -\int_{\Omega} \mathcal{Z}^{\alpha} \nabla \Delta c \mathcal{Z}^{\alpha} \nabla c d x \\
= & -\int_{\partial \Omega} \mathcal{Z}^{\alpha} \Delta c \mathcal{Z}^{\alpha} \nabla c \cdot \nu d \sigma+\int_{\Omega}\left|\operatorname{div}\left(\mathcal{Z}^{\alpha} \nabla c\right)\right|^{2} d x \\
& +\int_{\Omega}\left[\mathcal{Z}^{\alpha}, \operatorname{div}\right] \nabla c \operatorname{div}\left(\mathcal{Z}^{\alpha} \nabla c\right) d x-\int_{\Omega}\left[\mathcal{Z}^{\alpha}, \nabla\right] \Delta c \cdot \mathcal{Z}^{\alpha} \nabla c d x .
\end{aligned}
$$

Hence, we have

$$
\begin{aligned}
& \frac{1}{2} \frac{d}{d t} \int_{\Omega}\left|\mathcal{Z}^{\alpha} \nabla c\right|^{2} d x+\int_{\Omega}\left|\operatorname{div}\left(\mathcal{Z}^{\alpha} \nabla c\right)\right|^{2} d x \\
= & -\int_{\Omega} \mathcal{Z}^{\alpha} \nabla(n c) \mathcal{Z}^{\alpha} \nabla c d x-\int_{\Omega} \mathcal{Z}^{\alpha} \nabla(u \cdot \nabla c) \mathcal{Z}^{\alpha} \nabla c d x+\int_{\partial \Omega} \mathcal{Z}^{\alpha} \Delta c \mathcal{Z}^{\alpha} \nabla c \cdot \nu d \sigma \\
& -\int_{\Omega}\left[\mathcal{Z}^{\alpha}, \operatorname{div}\right] \nabla c \operatorname{div}\left(\mathcal{Z}^{\alpha} \nabla c\right) d x+\int_{\Omega}\left[\mathcal{Z}^{\alpha}, \nabla\right] \Delta c \cdot \mathcal{Z}^{\alpha} \nabla c d x .
\end{aligned}
$$

First, similar to Lemma 3.6, we can easily arrive at

$$
\begin{aligned}
& \left|\int_{0}^{t} \int_{\Omega}\left[\mathcal{Z}^{\alpha}, \operatorname{div}\right] \nabla c \operatorname{div}\left(\mathcal{Z}^{\alpha} \nabla c\right) d x d \tau-\int_{0}^{t} \int_{\Omega}\left[\mathcal{Z}^{\alpha}, \nabla\right] \Delta c \cdot \mathcal{Z}^{\alpha} \nabla c d x d \tau\right| \\
\leq & \delta \int_{0}^{t}\|\nabla \Delta c\|_{\mathcal{H}^{m-1}}^{2} d \tau+\delta \int_{0}^{t}\left\|\operatorname{div}\left(\mathcal{Z}^{\alpha} \nabla c\right)\right\|^{2} d \tau
\end{aligned}
$$




$$
+D_{\delta} D_{m+2}\left(\int_{0}^{t}\left\|\nabla^{2} c\right\|_{\mathcal{H}^{m-1}}^{2} d \tau+\int_{0}^{t}\|\nabla c\|_{\mathcal{H}^{m}}^{2} d \tau\right)
$$

Next, using Lemma 2.2 and Young's inequality, we have

$$
\begin{aligned}
& \left|\int_{0}^{t} \int_{\Omega} \mathcal{Z}^{\alpha} \nabla(n c) \mathcal{Z}^{\alpha} \nabla c d x d \tau\right| \\
\leq & \delta \int_{0}^{t}\|\nabla n\|_{\mathcal{H}^{m}}^{2} d \tau+D_{\delta} D_{m+2} P(M(t)) \int_{0}^{t}\left(\|c\|_{\mathcal{H}^{m}}^{2}+\|n\|_{\mathcal{H}^{m}}^{2}+\|\nabla c\|_{\mathcal{H}^{m}}^{2}\right) d \tau .
\end{aligned}
$$

Now, we turn to estimate the boundary term in (3.74). Like in Lemma 3.6, when $\left|\alpha_{0}\right|=m-1$ or $\left|\alpha_{13}\right| \neq 0$, this term vanishes. Thus, integrating by parts along the boundary lead to

$$
\left|\int_{0}^{t} \int_{\partial \Omega} \mathcal{Z}^{\alpha} \Delta c \mathcal{Z}^{\alpha} \nabla c \cdot \nu d \sigma d \tau\right| \leq \int_{0}^{t}\left|\partial_{t}^{\alpha_{0}} Z_{y}^{\beta} \Delta c\right|_{L^{2}(\partial \Omega)}\left|\mathcal{Z}^{\alpha} \nabla c \cdot \nu\right|_{H^{1}(\partial \Omega)} d \tau,
$$

where $|\beta|=m-\left|\alpha_{0}\right|-1$. By virtue of Lemma 2.3, we arrive at

$$
\left|\partial_{t}^{\alpha_{0}} Z_{y}^{\beta} \Delta c\right|_{L^{2}(\partial \Omega)} \leq D\left(\|\nabla \Delta c\|_{\mathcal{H}^{m-1}}^{\frac{1}{2}}+\|\Delta c\|_{\mathcal{H}^{m-1}}^{\frac{1}{2}}\right)\|\Delta c\|_{\mathcal{H}^{m-1}}^{\frac{1}{2}} .
$$

Based on the boundary condition (1.10) and Lemma 2.3, we can deduce that

$$
\left|\mathcal{Z}^{\alpha} \nabla c \cdot \nu\right|_{H^{1}(\partial \Omega)} \leq D\left(\left\|\nabla^{2} c\right\|_{\mathcal{H}^{m}}^{\frac{1}{2}}+\|\nabla c\|_{\mathcal{H}^{m}}^{\frac{1}{2}}\right)\|\nabla c\|_{\mathcal{H}^{m}}^{\frac{1}{2}}
$$

Combining (3.77)-(3.79) and using Young's inequality, we can get

$$
\begin{aligned}
\left|\int_{0}^{t} \int_{\partial \Omega} \mathcal{Z}^{\alpha} \Delta c \mathcal{Z}^{\alpha} \nabla c \cdot \nu d \sigma d \tau\right| \leq & \delta \int_{0}^{t}\|\nabla \Delta c\|_{\mathcal{H}^{m-1}}^{2} d \tau+\delta \int_{0}^{t}\left\|\nabla^{2} c\right\|_{\mathcal{H}^{m}}^{2} d \tau \\
& +D_{\delta} D_{m+2} \int_{0}^{t}\left(\|\nabla c\|_{\mathcal{H}^{m}}^{2}+\left\|\nabla^{2} c\right\|_{\mathcal{H}^{m-1}}^{2}\right) d \tau .
\end{aligned}
$$

Finally, we deal with the term involving $u$ in (3.74). By using integration by parts, it is easy to deduce that

$$
\begin{aligned}
& \int_{\Omega} \mathcal{Z}^{\alpha} \nabla(u \cdot \nabla c) \mathcal{Z}^{\alpha} \nabla c d x \\
= & \int_{\partial \Omega} \mathcal{Z}^{\alpha}(u \cdot \nabla c) \mathcal{Z}^{\alpha} \nabla c \cdot \nu d x+\int_{\Omega} \mathcal{Z}^{\alpha}(u \cdot \nabla c) \operatorname{div}\left(\mathcal{Z}^{\alpha} \nabla c\right) d x \\
& +\int_{\Omega}\left[\mathcal{Z}^{\alpha}, \nabla\right](u \cdot \nabla c) \mathcal{Z}^{\alpha} \nabla c d x .
\end{aligned}
$$

Similar to (3.60), we can integrate by parts along the boundary to deduce that

$$
\left|\int_{\partial \Omega} \mathcal{Z}^{\alpha}(u \cdot \nabla c) \mathcal{Z}^{\alpha} \nabla c \cdot \nu d x\right| \leq D\left|\partial_{t}^{\alpha_{0}} Z_{y}^{\beta}(u \cdot \nabla c)\right|_{L^{2}(\partial \Omega)}\left|\mathcal{Z}^{\alpha} \nabla c \cdot \nu\right|_{H^{1} \partial \Omega},
$$

where $|\beta|=m-\left|\alpha_{0}\right|-1$. Applying Lemma 2.3, we arrive at

$$
\begin{aligned}
\left|\partial_{t}^{\alpha_{0}} Z_{y}^{\beta}(u \cdot \nabla c)\right|_{L^{2}(\partial \Omega)} & \leq D\left(\|\nabla(u \cdot \nabla c)\|_{\mathcal{H}^{m-1}}^{\frac{1}{2}}+\|u \cdot \nabla c\|_{\mathcal{H}^{m-1}}^{\frac{1}{2}}\right)\|u \cdot \nabla c\|_{\mathcal{H}^{m-1}}^{\frac{1}{2}} \\
\left|\mathcal{Z}^{\alpha} \nabla c \cdot \nu\right|_{H^{1} \partial \Omega} & \leq D\left(\left\|\nabla^{2} c\right\|_{\mathcal{H}^{m}}^{\frac{1}{2}}+\|\nabla c\|_{\mathcal{H}^{m}}^{\frac{1}{2}}\right)\|\nabla c\|_{\mathcal{H}^{m}}^{\frac{1}{2}}
\end{aligned}
$$


By using Lemma 2.2 and Young's inequality, we obtain that

$$
\begin{aligned}
& \left|\int_{0}^{t} \int_{\partial \Omega} \mathcal{Z}^{\alpha}(u \cdot \nabla c) \mathcal{Z}^{\alpha} \nabla c \cdot \nu d x d \tau\right| \\
& \leq \delta \int_{0}^{t}\left\|\nabla^{2} c\right\|_{\mathcal{H}^{m}}^{2} d \tau+(1+P(M(t))) \\
& \quad \times \int_{0}^{t}\left(\|\nabla c\|_{\mathcal{H}^{m}}^{2}+\left\|\nabla^{2} c\right\|_{\mathcal{H}^{m-1}}^{2}+\|u\|_{\mathcal{H}^{m-1}}^{2}+\|\nabla u\|_{\mathcal{H}^{m-1}}^{2}\right) d \tau .
\end{aligned}
$$

In view of Young's inequality, we can get

$$
\begin{aligned}
& \left|\int_{0}^{t} \int_{\Omega} \mathcal{Z}^{\alpha}(u \cdot \nabla c) \operatorname{div}\left(\mathcal{Z}^{\alpha} \nabla c\right) d x d \tau+\int_{0}^{t} \int_{\Omega}\left[\mathcal{Z}^{\alpha}, \nabla\right](u \cdot \nabla c) \mathcal{Z}^{\alpha} \nabla c d x d \tau\right| \\
& \leq \delta \int_{0}^{t}\left\|\operatorname{div}\left(\mathcal{Z}^{\alpha} \nabla c\right)\right\|^{2} d \tau+D(1+P(M(t))) \int_{0}^{t}\left(\|\nabla c\|_{\mathcal{H}^{m}}^{2}+\left\|\nabla^{2} c\right\|_{\mathcal{H}^{m-1}}^{2}\right. \\
& \left.\quad+\|u\|_{\mathcal{H}^{m}}^{2}+\|\nabla u\|_{\mathcal{H}^{m-1}}^{2}\right) d \tau
\end{aligned}
$$

The combination of (3.83) and (3.84) yields that

$$
\begin{aligned}
& \left|\int_{0}^{t} \int_{\Omega} \mathcal{Z}^{\alpha} \nabla(u \cdot \nabla c) \mathcal{Z}^{\alpha} \nabla c d x d \tau\right| \\
& \leq \delta \int_{0}^{t}\left\|\operatorname{div}\left(\mathcal{Z}^{\alpha} \nabla c\right)\right\|^{2} d \tau+\delta \int_{0}^{t}\left\|\nabla^{2} c\right\|_{\mathcal{H}^{m}}^{2} d \tau+D_{m+2}(1+P(M(t))) \\
& \quad \times \int_{0}^{t}\left(\|\nabla c\|_{\mathcal{H}^{m}}^{2}+\left\|\nabla^{2} c\right\|_{\mathcal{H}^{m-1}}^{2}+\|u\|_{\mathcal{H}^{m}}^{2}+\|\nabla u\|_{\mathcal{H}^{m-1}}^{2}\right) d \tau
\end{aligned}
$$

Consequently, based on (3.68), (3.75), (3.76), (3.80), (3.85) and the inductive assumption yield (3.69). Therefore, we complete the proof of Lemma 3.7

3.3. Conormal Energy Estimates for $\Delta n$ and $\Delta c$. In this subsection, we can establish some uniform estimates for $\|\Delta n\|_{\mathcal{H}^{m-1}}$ and $\|\Delta c\|_{\mathcal{H}^{m-1}}$. We have the following uniform estimate with respect to $n$.

Lemma 3.8. For every $m \geq 1$, a smooth solution of the problem (1.5)-(1.11) satisfies the estimate

$$
\begin{aligned}
\sup _{0 \leq \tau \leq t}\|\Delta n\|_{\mathcal{H}^{m-1}}^{2}+\int_{0}^{t}\|\nabla \Delta n\|_{\mathcal{H}^{m-1}}^{2} d \tau \\
\leq D_{m+2}\left\{\left\|\Delta n_{0}\right\|_{\mathcal{H}^{m-1}}^{2}+(1+P(M(t))) \int_{0}^{t}\left(\|(n, c, u)\|_{\mathcal{H}^{m-1}}^{2}\right.\right. \\
\left.\left.\quad+\|\nabla(n, u)\|_{\mathcal{H}^{m-1}}^{2}+\|\nabla c\|_{\mathcal{H}^{m}}^{2}+\left\|\nabla^{2}(n, c)\right\|_{\mathcal{H}^{m-1}}^{2}\right) d \tau\right\} .
\end{aligned}
$$

Proof. Applying $\nabla$ to the equation (1.5) gives

$$
\nabla n_{t}+\nabla(u \cdot \nabla n)=\nabla \Delta n-\nabla(n \Delta c)-\nabla(\nabla n \cdot \nabla c) .
$$

By multiplying (3.87) by $\nabla \Delta n$ and integrating over $\Omega$, we obtain that

$$
-\int_{\Omega} \nabla n_{t} \cdot \nabla \Delta n d x+\int_{\Omega}|\nabla \Delta n|^{2} d x
$$




$$
\begin{aligned}
= & \int_{\Omega} \nabla(u \cdot \nabla n) \cdot \nabla \Delta n d x+\int_{\Omega} \nabla(n \Delta c) \cdot \nabla \Delta n d x \\
& +\int_{\Omega} \nabla(\nabla n \cdot \nabla c) \cdot \nabla \Delta n d x .
\end{aligned}
$$

By using the integration by parts and the boundary condition (1.10), we get

$$
-\int_{\Omega} \nabla n_{t} \cdot \nabla \Delta n d x=\frac{1}{2} \frac{d}{d t} \int_{\Omega}|\Delta n|^{2} d x
$$

In view of Young's inequality, we obtain that

$$
\begin{aligned}
& \left|\int_{0}^{t} \int_{\Omega} \nabla(u \cdot \nabla n) \cdot \nabla \Delta n-\nabla(n \Delta c) \cdot \nabla \Delta n-\nabla(\nabla n \cdot \nabla c) \cdot \nabla \Delta n d x d \tau\right| \\
\leq & \delta \int_{0}^{t}\|\nabla \Delta n\|^{2} d \tau+D_{\delta} M(t) \int_{0}^{t}\left(\|n\|^{2}+\|\nabla n\|^{2}+\left\|\nabla^{2} n\right\|^{2}\right) d \tau
\end{aligned}
$$

where $\delta$ is a small enough constant. Therefore, based on (3.88)-(3.90), (3.86) holds for $m=1$.

Now, we turn to do higher order uniform estimates. Assume that (3.86) has been proved for $|\alpha| \leq m-2$, we need to show that it holds for $|\alpha|=m-1$. By applying $\mathcal{Z}^{\alpha}$ with $|\alpha|=m-1$ to (3.87), we obtain that

$$
\mathcal{Z}^{\alpha} \nabla n_{t}+\mathcal{Z}^{\alpha} \nabla(u \cdot \nabla n)=\mathcal{Z}^{\alpha} \nabla \Delta n-\mathcal{Z}^{\alpha} \nabla(n \Delta c)-\mathcal{Z}^{\alpha} \nabla(\nabla n \cdot \nabla c) .
$$

Multiplying (3.91) by $\nabla \mathcal{Z}^{\alpha} \Delta n$, we get

$$
\begin{aligned}
& -\int_{\Omega} \mathcal{Z}^{\alpha} \nabla n_{t} \cdot \nabla \mathcal{Z}^{\alpha} \Delta n d x+\int_{\Omega} \mathcal{Z}^{\alpha} \nabla \Delta n \cdot \nabla \mathcal{Z}^{\alpha} \Delta n d x \\
= & \int_{\Omega} \mathcal{Z}^{\alpha} \nabla(u \cdot \nabla n) \cdot \nabla \mathcal{Z}^{\alpha} \Delta n d x+\int_{\Omega} \mathcal{Z}^{\alpha} \nabla(n \Delta c) \cdot \nabla \mathcal{Z}^{\alpha} \Delta n d x \\
& +\int_{\Omega} \mathcal{Z}^{\alpha} \nabla(\nabla n \cdot \nabla c) \cdot \nabla \mathcal{Z}^{\alpha} \Delta n d x .
\end{aligned}
$$

Due to integration by parts, we have

$$
\begin{aligned}
-\int_{\Omega} \mathcal{Z}^{\alpha} \nabla n_{t} \cdot \nabla \mathcal{Z}^{\alpha} \Delta n d x= & \frac{1}{2} \frac{d}{d t} \int_{\Omega}\left|\mathcal{Z}^{\alpha} \Delta n\right|^{2} d x-\int_{\partial \Omega} \nu \cdot \mathcal{Z}^{\alpha} \nabla n_{t}^{\epsilon} \mathcal{Z}^{\alpha} \Delta n d \sigma \\
& -\int_{\Omega}\left[\mathcal{Z}^{\alpha}, \nabla \cdot\right] \nabla n_{t} \mathcal{Z}^{\alpha} \Delta n d x \\
\int_{\Omega} \mathcal{Z}^{\alpha} \nabla \Delta n \cdot \nabla \mathcal{Z}^{\alpha} \Delta n d x= & \int_{\Omega}\left|\nabla \mathcal{Z}^{\alpha} \Delta n\right|^{2} d x+\int_{\Omega}\left[\mathcal{Z}^{\alpha}, \nabla\right] \Delta n \cdot \nabla \mathcal{Z}^{\alpha} \Delta n d x .
\end{aligned}
$$

Hence, from (3.93) and (3.94), we get

$$
\begin{aligned}
& \frac{1}{2} \frac{d}{d t} \int_{\Omega}\left|\mathcal{Z}^{\alpha} \Delta n\right|^{2} d x+\int_{\Omega}\left|\nabla \mathcal{Z}^{\alpha} \Delta n\right|^{2} d x \\
= & \int_{\Omega} \mathcal{Z}^{\alpha} \nabla(u \cdot \nabla n) \cdot \nabla \mathcal{Z}^{\alpha} \Delta n d x+\int_{\Omega} \mathcal{Z}^{\alpha} \nabla(n \Delta c) \cdot \nabla \mathcal{Z}^{\alpha} \Delta n d x \\
& +\int_{\partial \Omega} \nu \cdot \mathcal{Z}^{\alpha} \nabla n_{t} \mathcal{Z}^{\alpha} \Delta n d \sigma+\int_{\Omega}\left[\mathcal{Z}^{\alpha}, \nabla \cdot\right] \nabla n_{t} \mathcal{Z}^{\alpha} \Delta n d x
\end{aligned}
$$




$$
-\int_{\Omega}\left[\mathcal{Z}^{\alpha}, \nabla\right] \Delta n \cdot \nabla \mathcal{Z}^{\alpha} \Delta n d x+\int_{\Omega} \mathcal{Z}^{\alpha} \nabla(\nabla n \cdot \nabla c) \cdot \nabla \mathcal{Z}^{\alpha} \Delta n d x .
$$

First, we deal with the boundary term on the right-hand side of (3.95). Note that when $\left|\alpha_{0}\right|=m-1$, this integral vanishes. Hence, we assume $\left|\alpha_{0}\right| \leq m-2$. It is easy to deduce that

$$
\left|\int_{0}^{t} \int_{\partial \Omega} \nu \cdot \mathcal{Z}^{\alpha} \nabla n_{t} \mathcal{Z}^{\alpha} \Delta n d \sigma d \tau\right| \leq \int_{0}^{t}\left|\nu \cdot \mathcal{Z}^{\alpha} \nabla n_{t}\right|_{L^{2}(\partial \Omega)}\left|\mathcal{Z}^{\alpha} \Delta n\right|_{L^{2}(\partial \Omega)} d \tau .
$$

Based on Lemma 2.3 and the boundary condition (1.10), we get

$$
\begin{aligned}
\left|\nu \cdot \mathcal{Z}^{\alpha} \nabla n_{t}\right|_{L^{2}(\partial \Omega)} & \leq D_{m}\left(\left\|\nabla^{2} n\right\|_{\mathcal{H}^{m-1}}^{\frac{1}{2}}+\|\nabla n\|_{\mathcal{H}^{m-1}}^{\frac{1}{2}}\right)\|\nabla n\|_{\mathcal{H}^{m-1}}^{\frac{1}{2}}, \\
\left|\mathcal{Z}^{\alpha} \Delta n\right|_{L^{2}(\partial \Omega)} & \leq D_{m}\left(\|\nabla \Delta n\|_{\mathcal{H}^{m-1}}^{\frac{1}{2}}+\|\Delta n\|_{\mathcal{H}^{m-1}}^{\frac{1}{2}}\right)\|\Delta n\|_{\mathcal{H}^{m-1}}^{\frac{1}{2}} .
\end{aligned}
$$

Therefore, in view of Young's inequality, we have

$$
\begin{aligned}
\left|\int_{0}^{t} \int_{\partial \Omega} \nu \cdot \mathcal{Z}^{\alpha} \nabla n_{t} \mathcal{Z}^{\alpha} \Delta n d \sigma d \tau\right| \leq & \delta \int_{0}^{t}\|\nabla \Delta n\|_{\mathcal{H}^{m-1}}^{2} d \tau+D_{\delta} D_{m} \int_{0}^{t}\left(\left\|\nabla^{2} n\right\|_{\mathcal{H}^{m-1}}^{2}\right. \\
& \left.+\|\nabla n\|_{\mathcal{H}^{m-1}}^{2}\right) d \tau
\end{aligned}
$$

Next, we can use Young's inequality to get the following estimate directly,

$$
\begin{aligned}
& \left|\int_{0}^{t} \int_{\Omega}\left[\mathcal{Z}^{\alpha}, \nabla\right] \Delta n \cdot \nabla \mathcal{Z}^{\alpha} \Delta n d x d \tau\right| \\
\leq & \delta \int_{0}^{t}\|\nabla \Delta n\|_{\mathcal{H}^{m-1}}^{2} d \tau+D_{\delta} \int_{0}^{t}\|\nabla \Delta n\|_{\mathcal{H}^{m-2}}^{2} d \tau .
\end{aligned}
$$

Also, it is easy to deduce that

$$
\left|\int_{0}^{t} \int_{\Omega}\left[\mathcal{Z}^{\alpha}, \nabla \cdot\right] \nabla n_{t} \mathcal{Z}^{\alpha} \Delta n d x d \tau\right| \leq D \int_{0}^{t}\left\|\nabla^{2} n\right\|_{\mathcal{H}^{m-1}}^{2} d \tau
$$

Furthermore, by virtue of Lemma 2.2 and Young's inequality, we obtain that

$$
\begin{aligned}
& \left|\int_{0}^{t} \int_{\Omega} \mathcal{Z}^{\alpha} \nabla(\nabla n \cdot \nabla c) \cdot \nabla \mathcal{Z}^{\alpha} \Delta n d x d \tau\right| \\
& \leq \delta \int_{0}^{t}\|\nabla \Delta n\|_{\mathcal{H}^{m-1}}^{2} d \tau+D_{\delta} P(M(t)) \int_{0}^{t}\left(\left\|\nabla^{2} n\right\|_{\mathcal{H}^{m-1}}^{2}+\|\nabla n\|_{\mathcal{H}^{m-1}}^{2}\right. \\
& \left.\quad+\left\|\nabla^{2} c\right\|_{\mathcal{H}^{m-1}}^{2}+\|\nabla c\|_{\mathcal{H}^{m-1}}^{2}\right) d \tau, \\
& \quad\left|\int_{0}^{t} \int_{\Omega} \mathcal{Z}^{\alpha} \nabla(u \cdot \nabla n) \cdot \nabla \mathcal{Z}^{\alpha} \Delta n d x d \tau\right| \\
& \leq \delta \int_{0}^{t}\|\nabla \Delta n\|_{\mathcal{H}^{m-1}}^{2} d \tau+D_{\delta} P(M(t)) \int_{0}^{t}\left(\|\nabla n\|_{\mathcal{H}^{m-1}}^{2}+\left\|\nabla^{2} n\right\|_{\mathcal{H}^{m-1}}^{2}\right. \\
& \left.\quad+\|\nabla u\|_{\mathcal{H}^{m-1}}^{2}+\|u\|_{\mathcal{H}^{m-1}}^{2}\right) d \tau .
\end{aligned}
$$

Finally, because we don't expect that $\|\nabla \Delta c\|_{\mathcal{H}^{m-1}}^{2}$ appear in the right-hand side of energy inequality, we deal with $\int_{\Omega} \mathcal{Z}^{\alpha} \nabla(n \Delta c) \cdot \nabla \mathcal{Z}^{\alpha} \Delta n d x$ with the help of the 
equation (1.6) and Lemma 2.2, We have

$$
\begin{aligned}
& \left|\int_{0}^{t} \int_{\Omega} \mathcal{Z}^{\alpha} \nabla(n \Delta c) \cdot \nabla \mathcal{Z}^{\alpha} \Delta n d x d \tau\right| \\
& \leq \delta \int_{0}^{t}\|\nabla \Delta n\|_{\mathcal{H}^{m-1}}^{2} d \tau+D_{\delta} P(M(t)) \int_{0}^{t}\left(\|(n, c, u)\|_{\mathcal{H}^{m-1}}^{2}\right. \\
& \left.\quad+\|\nabla(n, u)\|_{\mathcal{H}^{m-1}}^{2}+\left\|\nabla^{2} c\right\|_{\mathcal{H}^{m-1}}^{2}+\|\nabla c\|_{\mathcal{H}^{m}}^{2}\right) d \tau .
\end{aligned}
$$

Consequently, the combination of (3.68), (3.97)-(3.102) and the inductive assumption yield (3.86). Therefore, we complete the proof of Lemma 3.8

Now, we turn to the estimate $\|\Delta c\|_{\mathcal{H}^{m-1}}$. We have

Lemma 3.9. For every $m \geq 1$, a smooth solution of the problem (1.5)-(1.11) satisfies the estimate

$$
\begin{aligned}
& \sup _{0 \leq \tau \leq t}\|\Delta c\|_{\mathcal{H}^{m-1}}^{2}+\int_{0}^{t}\|\nabla \Delta c\|_{\mathcal{H}^{m-1}}^{2} d \tau \\
& \leq D_{m+2}\left\{\left\|\Delta c_{0}\right\|_{\mathcal{H}^{m-1}}^{2}+(1+P(M(t))) \int_{0}^{t}\left(\|(n, c, u)\|_{\mathcal{H}^{m-1}}^{2}\right.\right. \\
& \left.\left.\quad+\|\nabla(n, c, u)\|_{\mathcal{H}^{m-1}}^{2}+\left\|\nabla^{2} c\right\|_{\mathcal{H}^{m-1}}^{2}\right) d \tau\right\}
\end{aligned}
$$

Proof. Applying $\nabla$ to the equation (1.6) gives

$$
\nabla c_{t}+\nabla(u \cdot \nabla c)=\nabla \Delta c-\nabla(n c) .
$$

By multiplying (3.104) by $\nabla \Delta c$ and integrating over $\Omega$, we get

$$
-\int_{\Omega} \nabla c_{t} \cdot \nabla \Delta c d x+\int_{\Omega}|\nabla \Delta c|^{2} d x=\int_{\Omega} \nabla(u \cdot \nabla c) \cdot \nabla \Delta c d x+\int_{\Omega} \nabla(n c) \cdot \nabla \Delta c d x .
$$

Integrating by parts and using the boundary condition (1.10) yield

$$
-\int_{\Omega} \nabla c_{t} \cdot \nabla \Delta c d x=\frac{1}{2} \frac{d}{d t} \int_{\Omega}|\Delta c|^{2} d x
$$

By virtue of Young's inequality, we get

$$
\begin{aligned}
& \left|\int_{0}^{t} \int_{\Omega} \nabla(u \cdot \nabla c) \cdot \nabla \Delta c-\nabla(n c) \cdot \nabla \Delta c d x d \tau\right| \\
\leq & \delta \int_{0}^{t}\|\nabla \Delta c\|^{2} d \tau+D_{\delta} M(t) \int_{0}^{t}\left(\|c\|^{2}+\|\nabla c\|^{2}+\left\|\nabla^{2} c\right\|^{2}\right) d \tau .
\end{aligned}
$$

Therefore, the combination of (3.105)-(3.107) implies that (3.103) holds for $m=1$.

Now, we show that (3.103) holds for $|\alpha| \leq m-1$. Assume that (3.103) is proved for $|\alpha| \leq m-2$, we need to prove it for $|\alpha|=m-1$. Applying $\mathcal{Z}^{\alpha}$ with $|\alpha|=m-1$ to (3.104) gives

$$
\mathcal{Z}^{\alpha} \nabla c_{t}+\mathcal{Z}^{\alpha} \nabla(u \cdot \nabla c)=\mathcal{Z}^{\alpha} \nabla \Delta c-\mathcal{Z}^{\alpha} \nabla(n c) .
$$


Multiplying (3.108) by $\nabla \mathcal{Z}^{\alpha} \Delta c$, we obtain that

$$
\begin{aligned}
& -\int_{\Omega} \mathcal{Z}^{\alpha} \nabla c_{t} \cdot \nabla \mathcal{Z}^{\alpha} \Delta c d x+\int_{\Omega} \mathcal{Z}^{\alpha} \nabla \Delta c \cdot \nabla \mathcal{Z}^{\alpha} \Delta c d x \\
= & \int_{\Omega} \mathcal{Z}^{\alpha} \nabla(u \cdot \nabla c) \cdot \nabla \mathcal{Z}^{\alpha} \Delta c d x+\int_{\Omega} \mathcal{Z}^{\alpha} \nabla(n c) \cdot \nabla \mathcal{Z}^{\alpha} \Delta c d x .
\end{aligned}
$$

By integrating by parts, we have

$$
\begin{aligned}
-\int_{\Omega} \mathcal{Z}^{\alpha} \nabla c_{t} \cdot \nabla \mathcal{Z}^{\alpha} \Delta c d x= & \frac{1}{2} \frac{d}{d t} \int_{\Omega}\left|\mathcal{Z}^{\alpha} \Delta c\right|^{2} d x-\int_{\partial \Omega} \nu \cdot \mathcal{Z}^{\alpha} \nabla c_{t}^{\epsilon} \mathcal{Z}^{\alpha} \Delta c d \sigma \\
& -\int_{\Omega}\left[\mathcal{Z}^{\alpha}, \nabla \cdot\right] \nabla c_{t} \mathcal{Z}^{\alpha} \Delta c d x \\
\int_{\Omega} \mathcal{Z}^{\alpha} \nabla \Delta c \cdot \nabla \mathcal{Z}^{\alpha} \Delta c d x= & \int_{\Omega}\left|\nabla \mathcal{Z}^{\alpha} \Delta c\right|^{2} d x+\int_{\Omega}\left[\mathcal{Z}^{\alpha}, \nabla\right] \Delta c \cdot \nabla \mathcal{Z}^{\alpha} \Delta c d x
\end{aligned}
$$

From (3.110) and (3.111), we get

$$
\begin{aligned}
& \frac{1}{2} \frac{d}{d t} \int_{\Omega}\left|\mathcal{Z}^{\alpha} \Delta c\right|^{2} d x+\int_{\Omega}\left|\nabla \mathcal{Z}^{\alpha} \Delta c\right|^{2} d x \\
= & \int_{\Omega} \mathcal{Z}^{\alpha} \nabla(u \cdot \nabla c) \cdot \nabla \mathcal{Z}^{\alpha} \Delta c d x+\int_{\Omega} \mathcal{Z}^{\alpha} \nabla(n c) \cdot \nabla \mathcal{Z}^{\alpha} \Delta c d x \\
& +\int_{\partial \Omega} \nu \cdot \mathcal{Z}^{\alpha} \nabla c_{t} \mathcal{Z}^{\alpha} \Delta c d \sigma+\int_{\Omega}\left[\mathcal{Z}^{\alpha}, \nabla \cdot\right] \nabla c_{t} \mathcal{Z}^{\alpha} \Delta c d x \\
& -\int_{\Omega}\left[\mathcal{Z}^{\alpha}, \nabla\right] \Delta c \cdot \nabla \mathcal{Z}^{\alpha} \Delta c d x .
\end{aligned}
$$

First, we estimate the boundary term in the right-hand side of (3.112). Note that when $\left|\alpha_{0}\right|=m-1$, this integral vanishes. Hence, we assume $\left|\alpha_{0}\right| \leq m-2$. It is easy to deduce that

$$
\left|\int_{0}^{t} \int_{\partial \Omega} \nu \cdot \mathcal{Z}^{\alpha} \nabla c_{t} \mathcal{Z}^{\alpha} \Delta c d \sigma d \tau\right| \leq \int_{0}^{t}\left|\nu \cdot \mathcal{Z}^{\alpha} \nabla c_{t}\right|_{L^{2}(\partial \Omega)}\left|\mathcal{Z}^{\alpha} \Delta c\right|_{L^{2}(\partial \Omega)} d \tau .
$$

Similar to (3.97), by virtue of Lemma 2.3, the boundary condition (1.10) and Young's inequality, we get

$$
\begin{aligned}
& \left|\int_{0}^{t} \int_{\partial \Omega} \nu \cdot \mathcal{Z}^{\alpha} \nabla c_{t} \mathcal{Z}^{\alpha} \Delta c d \sigma d \tau\right| \\
\leq & \delta \int_{0}^{t}\|\nabla \Delta c\|_{\mathcal{H}^{m-1}}^{2} d \tau+D_{\delta} D_{m} \int_{0}^{t}\left(\left\|\nabla^{2} c\right\|_{\mathcal{H}^{m-1}}^{2}+\|\nabla c\|_{\mathcal{H}^{m-1}}^{2}\right) d \tau .
\end{aligned}
$$

Next, by using Young's inequality, we can easy obtain

$$
\begin{aligned}
& \left|\int_{0}^{t} \int_{\Omega}\left[\mathcal{Z}^{\alpha}, \nabla\right] \Delta c \cdot \nabla \mathcal{Z}^{\alpha} \Delta c d x d \tau\right| \\
\leq & \delta \int_{0}^{t}\|\nabla \Delta c\|_{\mathcal{H}^{m-1}}^{2} d \tau+D_{\delta} \int_{0}^{t}\|\nabla \Delta c\|_{\mathcal{H}^{m-2}}^{2} d \tau .
\end{aligned}
$$

Also, it is easy to deduce that

$$
\left|\int_{0}^{t} \int_{\Omega}\left[\mathcal{Z}^{\alpha}, \nabla \cdot\right] \nabla c_{t} \mathcal{Z}^{\alpha} \Delta c d x d \tau\right| \leq D \int_{0}^{t}\left\|\nabla^{2} c\right\|_{\mathcal{H}^{m-1}}^{2} d \tau
$$


Finally, by virtue of Lemma 2.2 and Young's inequality, we obtain that

$$
\begin{aligned}
& \left|\int_{0}^{t} \int_{\Omega} \mathcal{Z}^{\alpha} \nabla(n c) \cdot \nabla \mathcal{Z}^{\alpha} \Delta c d x d \tau\right| \\
& \leq \delta \int_{0}^{t}\|\nabla \Delta c\|_{\mathcal{H}^{m-1}}^{2} d \tau+D_{\delta} P(M(t)) \int_{0}^{t}\left(\|n\|_{\mathcal{H}^{m-1}}^{2}\right. \\
& \left.\quad+\|\nabla n\|_{\mathcal{H}^{m-1}}^{2}+\|c\|_{\mathcal{H}^{m-1}}^{2}+\|\nabla c\|_{\mathcal{H}^{m-1}}^{2}\right) d \tau, \\
& \quad\left|\int_{0}^{t} \int_{\Omega} \mathcal{Z}^{\alpha} \nabla(u \cdot \nabla c) \cdot \nabla \mathcal{Z}^{\alpha} \Delta c d x d \tau\right| \\
& \leq \delta \int_{0}^{t}\|\nabla \Delta c\|_{\mathcal{H}^{m-1}}^{2} d \tau+D_{\delta} P(M(t)) \int_{0}^{t}\left(\|\nabla c\|_{\mathcal{H}^{m-1}}^{2}\right. \\
& \left.\quad+\left\|\nabla^{2} c\right\|_{\mathcal{H}^{m-1}}^{2}+\|\nabla u\|_{\mathcal{H}^{m-1}}^{2}+\|u\|_{\mathcal{H}^{m-1}}^{2}\right) d \tau .
\end{aligned}
$$

Consequently, based on (3.68), (3.113)-(3.117) and the inductive assumption, we can complete the proof of Lemma 3.9 .

It follows from Lemma 3.4 to Lemma 3.9 where $\delta>0$ is suitably small, that

$$
\begin{aligned}
& \sup _{0 \leq \tau \leq t}\left(\|(n, c, u)\|_{\mathcal{H}^{m}}^{2}+\|\nabla n\|_{\mathcal{H}^{m-1}}^{2}+\|\nabla c\|_{\mathcal{H}^{m}}^{2}+\|\Delta(n, c)\|_{\mathcal{H}^{m-1}}^{2}\right) \\
& +\epsilon \int_{0}^{t}\|\nabla u\|_{\mathcal{H}^{m}}^{2} d \tau+\int_{0}^{t}\left(\|\nabla n\|_{\mathcal{H}^{m}}^{2}+\|\Delta c\|_{\mathcal{H}^{m}}^{2}+\|\nabla \Delta(n, c)\|_{\mathcal{H}^{m-1}}^{2}\right) d \tau \\
& \leq D_{m+2}\left\{\left\|\left(n_{0}, c_{0}, u_{0}\right)\right\|_{\mathcal{H}^{m}}^{2}+\left\|\nabla n_{0}\right\|_{\mathcal{H}^{m-1}}^{2}+\left\|\nabla c_{0}\right\|_{\mathcal{H}^{m}}^{2}+\left\|\Delta\left(n_{0}, c_{0}\right)\right\|_{\mathcal{H}^{m-1}}^{2}\right. \\
& \quad+\int_{0}^{t}\left(\left\|\nabla^{2} p_{1}\right\|_{\mathcal{H}^{m-1}}\|u\|_{\mathcal{H}^{m}}+\epsilon^{-1}\left(\left\|\nabla p_{2}\right\|_{\mathcal{H}^{m-1}}^{2}+\left\|p_{2}\right\|_{\mathcal{H}^{m-1}}^{2}\right)\right) d \tau \\
& +(1+P(M(t))) \int_{0}^{t}\left(\|(n, c, u)\|_{\mathcal{H}^{m}}^{2}+\|\nabla(n, u)\|_{\mathcal{H}^{m-1}}^{2}+\|\nabla c\|_{\mathcal{H}^{m}}^{2}\right. \\
& \left.\left.+\|\Delta(n, c)\|_{\mathcal{H}^{m-1}}^{2}\right) d \tau+\int_{0}^{t}\|n \nabla \phi\|_{\mathcal{H}^{m}}^{2} d \tau\right\} .
\end{aligned}
$$

3.4. Normal Derivative Estimates. In this subsection, we provide the estimate for $\|\nabla u\|_{\mathcal{H}^{m-1}}$. Note that

$$
\left\|\chi \partial_{y^{i}} u\right\|_{\mathcal{H}^{m-1}} \leq D\|u\|_{\mathcal{H}^{m}} \quad \text { for } \quad i=1,2,
$$

it suffices to estimate $\left\|\chi \partial_{\nu} u\right\|_{\mathcal{H}^{m-1}}$, where $\chi$ is compactly supported in one of the $\Omega_{i}$ and with value one in a vicinity of the boundary. We shall thus use the local coordinates (1.23). Due to (3.21), we immediately obtain that

$$
\left\|\chi \partial_{\nu} u \cdot \nu\right\|_{\mathcal{H}^{m-1}} \leq D_{m}\|u\|_{\mathcal{H}^{m}}
$$

Thus, it remains to estimate $\left\|\chi \Pi \partial_{\nu} u\right\|_{\mathcal{H}^{m-1}}$. We define

$$
\eta:=\chi \Pi\left(\left(\nabla u+(\nabla u)^{t}\right) \nu\right)+2 \zeta \chi \Pi u .
$$

In view of the boundary condition (1.11), we have

$$
\eta=0 \quad \text { on } \quad \partial \Omega .
$$


Moreover, since $\eta$ have another form in the vicinity of the boundary $\partial \Omega$ :

$$
\eta=\chi \Pi \partial_{\nu} u+\chi \Pi(\nabla(u \cdot \nu)-\nabla \nu \cdot u-u \times(\nabla \times \nu)+2 \zeta u),
$$

we easily get that

$$
\begin{aligned}
\left\|\chi \Pi \partial_{\nu} u\right\|_{\mathcal{H}^{m-1}} & \leq D_{m+1}\left(\|\eta\|_{\mathcal{H}^{m-1}}+\|u\|_{\mathcal{H}^{m}}+\left\|\partial_{\nu} u \cdot \nu\right\|_{\mathcal{H}^{m}}\right) \\
& \leq D_{m+1}\left(\|\eta\|_{\mathcal{H}^{m-1}}+\|u\|_{\mathcal{H}^{m}}\right)
\end{aligned}
$$

Hence, it suffices to estimate $\|\eta\|_{\mathcal{H}^{m-1}}$. We have the following estimates for $\eta$.

Lemma 3.10. For every $m \geq 1$, we have

$$
\begin{aligned}
& \sup _{0 \leq \tau \leq t}\|\eta\|_{\mathcal{H}^{m-1}}^{2}+\epsilon \int_{0}^{t}\|\nabla \eta\|_{\mathcal{H}^{m-1}}^{2} d \tau \\
& \leq D_{m+2}\left\{\left\|u_{0}\right\|_{\mathcal{H}^{m}}^{2}+\left\|\nabla u_{0}\right\|_{\mathcal{H}^{m-1}}^{2}+\epsilon^{-1} \int_{0}^{t}\left\|\nabla p_{2}\right\|_{\mathcal{H}^{m-1}}^{2} d \tau+\epsilon^{2} \int_{0}^{t}\|\nabla u\|_{\mathcal{H}^{m}}^{2} d \tau\right. \\
& \quad+\int_{0}^{t}\left(\left\|\nabla^{2} p_{1}\right\|_{\mathcal{H}^{m-1}}+\left\|\nabla p_{1}\right\|_{\mathcal{H}^{m-1}}\right)\|\eta\|_{\mathcal{H}^{m-1}} d \tau+\int_{0}^{t}\left\|\nabla^{2} p_{1}\right\|_{\mathcal{H}^{m-1}}\|u\|_{\mathcal{H}^{m}} d \tau \\
& \quad+\int_{0}^{t}\|\nabla(n \nabla \phi)\|_{\mathcal{H}^{m-1}}\|\eta\|_{\mathcal{H}^{m-1}} d \tau+\int_{0}^{t}\|n \nabla \phi\|_{\mathcal{H}^{m-1}}\|u\|_{\mathcal{H}^{m}} d \tau \\
& \left.+(1+P(M(t))) \int_{0}^{t}\left(\|u\|_{\mathcal{H}^{m}}^{2}+\|\nabla u\|_{\mathcal{H}^{m-1}}^{2}\right) d \tau\right\} .
\end{aligned}
$$

Proof. Setting $\mathcal{N}=\nabla u$, we get from (1.7) that

$$
\mathcal{N}_{t}-\epsilon \Delta \mathcal{N}+u \cdot \nabla \mathcal{N}=-\mathcal{N}^{2}-\nabla^{2} p+\nabla(n \nabla \phi) .
$$

Hence, $\eta$ solves the equation

$$
\eta_{t}-\epsilon \Delta \eta+u \cdot \nabla \eta=F-2 \chi \Pi\left(\nabla^{2} p \nu\right),
$$

where $F:=F^{b}+F^{\chi}+F^{\kappa}$ with

$$
\begin{aligned}
F^{b}:= & -\chi \Pi\left(\left((\nabla u)^{2}+\left((\nabla u)^{t}\right)^{2}\right) \nu\right)-2 \zeta \chi \Pi \nabla p-\chi \Pi\left((\nabla(n \nabla \phi))^{2}\right. \\
& \left.\left.-\left((\nabla(n \nabla \phi))^{t}\right)^{2}\right) \nu\right), \\
F^{\chi}:= & -\epsilon \Delta \chi(\Pi S u \nu+2 \zeta \Pi u)-2 \epsilon \nabla \chi \cdot \nabla(\Pi S u \nu+2 \zeta \Pi u)+u^{\epsilon} \cdot \nabla \chi \Pi(S u \nu+2 \zeta u), \\
F^{\kappa}:= & \chi(u \cdot \nabla \Pi)(S u \nu+2 \zeta u)+\chi \Pi(S u(u \cdot \nabla) \nu)-\epsilon \chi(\Delta \Pi)(S u \nu+2 \zeta u) \\
& -2 \epsilon \chi \nabla \Pi \cdot \nabla(S u \nu+2 \zeta u)-\epsilon \chi \Pi(S u \Delta \nu+2 \nabla S u \cdot \nabla \nu) .
\end{aligned}
$$

Let us start with the case of $m=1$. Due to (1.8), we get

$$
\frac{1}{2} \frac{d}{d t}\|\eta\|^{2}+\epsilon \int_{\Omega}|\nabla \eta|^{2} d x=\int_{\Omega} F \cdot \eta d x-\int_{\Omega} \chi \Pi\left(\nabla^{2} p \nu\right) \cdot \eta d x .
$$

Now we estimate the right-hand side terms of (3.123). In view of Lemma 2.2 we easily get

$$
\int_{0}^{t}\left\|F^{b}\right\|_{\mathcal{H}^{m-1}}^{2} d \tau \leq D_{m} P(M(t)) \int_{0}^{t}\|\nabla u\|_{\mathcal{H}^{m-1}}^{2} d \tau+D \int_{0}^{t}\|\nabla(n \nabla \phi)\|_{\mathcal{H}^{m-1}}^{2} d \tau
$$




$$
\begin{gathered}
+D_{m} \int_{0}^{t}\|\nabla p\|_{\mathcal{H}^{m-1}}^{2} d \tau \\
\int_{0}^{t}\left\|F^{\chi}\right\|_{\mathcal{H}^{m-1}}^{2} d \tau \leq D_{m+1}(1+P(M(t))) \int_{0}^{t}\|u\|_{\mathcal{H}^{m}}^{2} d \tau+D \epsilon^{2} \int_{0}^{t}\|\nabla u\|_{\mathcal{H}^{m}}^{2} d \tau \\
\int_{0}^{t}\left\|F^{\kappa}\right\|_{\mathcal{H}^{m-1}}^{2} d \tau \leq D_{m+2}(1+P(M(t))) \int_{0}^{t}\left(\|u\|_{\mathcal{H}^{m}}^{2}+\|\nabla u\|_{\mathcal{H}^{m-1}}^{2}\right) d \tau \\
+D \epsilon^{2} \int_{0}^{t}\left(\|\nabla u\|_{\mathcal{H}^{m-1}}^{2}+\left\|\chi \nabla^{2} u\right\|_{\mathcal{H}^{m-1}}^{2}\right) d \tau
\end{gathered}
$$

Next, we estimate the term involving the pressure $p$ in (3.123). By recalling that $p=p_{1}+p_{2}$, we get

$$
\left|\int_{0}^{t} \int_{\Omega} \chi \Pi\left(\nabla^{2} p \nu\right) \cdot \eta d x d \tau\right| \leq D \int_{0}^{t}\left\|\nabla^{2} p_{1}\right\|\|\eta\| d \tau+\left|\int_{0}^{t} \int_{\Omega} \chi \Pi\left(\nabla^{2} p_{2} \nu\right) \cdot \eta d x d \tau\right| .
$$

Since $\eta=0$ on the boundary, we can integrate by parts the last term in (3.127) to obtain

$$
\left|\int_{0}^{t} \int_{\Omega} \chi \Pi\left(\nabla^{2} p_{2} \nu\right) \cdot \eta d x d \tau\right| \leq D \int_{0}^{t}\left\|\nabla p_{2}\right\|(\|\nabla \eta\|+\|\eta\|) d \tau
$$

Therefore, putting (3.124)-(3.128) into (3.123) and using Young's inequality, we obtain that

$$
\begin{aligned}
& \sup _{0 \leq \tau \leq t}\|\eta\|^{2}+\epsilon \int_{0}^{t}\|\nabla \eta\|^{2} d \tau \\
\leq & D_{3}\left\{\left\|\eta_{0}\right\|^{2}+\epsilon^{-1} \int_{0}^{t}\left\|\nabla p_{2}\right\|^{2} d \tau+\int_{0}^{t}\left(\left\|\nabla^{2} p_{1}\right\|+\|\nabla p\|+\|\nabla(n \nabla \phi)\|\right)\|\eta\| d \tau\right. \\
& \left.+\delta \epsilon^{2} \int_{0}^{t}\left\|\chi \nabla^{2} u\right\|^{2} d \tau+(1+P(M(t))) \int_{0}^{t}\left(\|u\|_{\mathcal{H}^{1}}^{2}+\|\nabla u\|^{2}\right) d \tau\right\}
\end{aligned}
$$

Due to (3.119) and (3.120), we get

$$
\epsilon\left\|\chi \nabla^{2} u\right\|_{\mathcal{H}^{m-1}} \leq D_{m+2} \epsilon\left(\|\nabla \eta\|_{\mathcal{H}^{m-1}}+\|\nabla u\|_{\mathcal{H}^{m}}+\|u\|_{\mathcal{H}^{m}}\right) .
$$

Furthermore, we have

$$
\begin{aligned}
& \sup _{0 \leq \tau \leq t}\|\eta\|^{2}+\epsilon \int_{0}^{t}\|\nabla \eta\|^{2} d \tau \\
\leq & D_{3}\left\{\left\|\eta_{0}\right\|^{2}+\epsilon^{-1} \int_{0}^{t}\left\|\nabla p_{2}\right\|^{2} d \tau+\int_{0}^{t}\left(\left\|\nabla^{2} p_{1}\right\|+\|\nabla p\|+\|\nabla(n \nabla \phi)\|\right)\|\eta\| d \tau\right. \\
& \left.+\delta \epsilon^{2} \int_{0}^{t}\|\nabla u\|_{\mathcal{H}^{1}}^{2} d \tau+(1+P(M(t))) \int_{0}^{t}\left(\|u\|_{\mathcal{H}^{1}}^{2}+\|\nabla u\|^{2}\right) d \tau\right\} .
\end{aligned}
$$

Hence, (3.121) holds for $m=1$.

Now we assume that Lemma 3.10 is true for $|\alpha| \leq m-2$ and let us consider the situation of $|\alpha|=m-1$. By applying $\mathcal{Z}^{\alpha}$ to (3.122), we have

$$
\mathcal{Z}^{\alpha} \eta_{t}-\epsilon \mathcal{Z}^{\alpha} \Delta \eta+u \cdot \nabla Z^{\alpha} \eta=\mathcal{Z}^{\alpha} F-\mathcal{Z}^{\alpha}\left(2 \chi \Pi\left(\nabla^{2} p \nu\right)\right)+\mathcal{C}_{4}
$$


where

$$
\mathcal{C}_{4}:=-\left[\mathcal{Z}^{\alpha}, u \cdot \nabla\right] \eta
$$

Multiplying (3.132) by $\mathcal{Z}^{\alpha} \eta^{\epsilon}$, we obtain that

$$
\begin{aligned}
\frac{1}{2} \frac{d}{d t}\left\|\mathcal{Z}^{\alpha} \eta\right\|^{2}= & \epsilon \int_{\Omega} \mathcal{Z}^{\alpha} \Delta \eta \cdot \mathcal{Z}^{\alpha} \eta d x-2 \int_{\Omega} \mathcal{Z}^{\alpha}\left(\chi \Pi\left(\nabla^{2} p \nu\right)\right) \cdot \mathcal{Z}^{\alpha} \eta d x \\
& +\int_{\Omega} \mathcal{Z}^{\alpha} F \cdot \mathcal{Z}^{\alpha} \eta d x+\int_{\Omega} \mathcal{C}_{4} \cdot \mathcal{Z}^{\alpha} \eta d x .
\end{aligned}
$$

First, let us estimate the viscous term. We observe that

$$
\begin{aligned}
& \epsilon \int_{0}^{t} \int_{\Omega} \mathcal{Z}^{\alpha} \partial_{i i} \eta \cdot \mathcal{Z}^{\alpha} \eta d x d \tau \\
= & -\epsilon \int_{0}^{t} \int_{\Omega}\left|\partial_{i} \mathcal{Z}^{\alpha} \eta\right|^{2} d x d \tau-\epsilon \int_{0}^{t} \int_{\Omega}\left[\mathcal{Z}^{\alpha}, \partial_{i}\right] \eta \cdot \partial_{i} \mathcal{Z}^{\alpha} \eta d x d \tau \\
& +\epsilon \int_{0}^{t} \int_{\Omega}\left[\mathcal{Z}^{\alpha}, \partial_{i}\right] \partial_{i} \eta \cdot \mathcal{Z}^{\alpha} \eta d x d \tau,
\end{aligned}
$$

where $i=1,2,3$. In order to estimate the last two terms in the right-hand side of (3.134), we use the structure of the commutator $\left[\mathcal{Z}^{\alpha}, \partial_{i}\right]$ and the expansion $\partial_{i}=\beta^{1} \partial_{y^{1}}+\beta^{2} \partial_{y^{2}}+\beta^{3} \partial_{y^{3}}$ in the local basis. We have the following expansion

$$
\left[\mathcal{Z}^{\alpha}, \partial_{i}\right] \eta=\sum_{\gamma,|\gamma| \leq|\alpha|-1} d_{\gamma} \partial_{z} \mathcal{Z}^{\gamma} \eta+\sum_{\beta,|\beta| \leq|\alpha|} d_{\beta} \mathcal{Z}^{\beta} \eta
$$

Thus, we have

$$
\begin{aligned}
& \epsilon\left|\int_{0}^{t} \int_{\Omega}\left[\mathcal{Z}^{\alpha}, \partial_{i}\right] \eta \cdot \partial_{i} \mathcal{Z}^{\alpha} \eta d x d \tau\right| \\
& \leq D_{m} \epsilon \int_{0}^{t}\left\|\nabla \mathcal{Z}^{m-1} \eta\right\|\left(\|\nabla \eta\|_{\mathcal{H}^{m-2}}+\|\eta\|_{\mathcal{H}^{m-1}}\right) d \tau \\
& \epsilon\left|\int_{0}^{t} \int_{\Omega}\left[\mathcal{Z}^{\alpha}, \partial_{i}\right] \partial_{i} \eta \cdot \mathcal{Z}^{\alpha} \eta d x d \tau\right| \\
& \leq D_{m} \epsilon\left\{\int_{0}^{t}\|\nabla \eta\|_{\mathcal{H}^{m-1}}\|\eta\|_{\mathcal{H}^{m-1}} d \tau+\sum_{|\gamma| \leq m-2}\left|\int_{0}^{t} \int_{\Omega} D_{\gamma} \partial_{z} \mathcal{Z}^{\gamma} \partial_{i} \eta \cdot \mathcal{Z}^{\alpha} \eta d x d \tau\right|\right\}
\end{aligned}
$$

Furthermore, by virtue of $\mathcal{Z}^{\alpha} \eta^{\epsilon}=0$ on $\partial \Omega$ and integration by parts, we obtain that

$$
\begin{aligned}
& \epsilon\left|\int_{0}^{t} \int_{\Omega}\left[\mathcal{Z}^{\alpha}, \partial_{i}\right] \partial_{i} \eta \cdot \mathcal{Z}^{\alpha} \eta d x d \tau\right| \\
& \leq D_{m+1} \epsilon \int_{0}^{t}\|\nabla \eta\|_{\mathcal{H}^{m-1}}\left(\|\eta\|_{\mathcal{H}^{m-1}}+\|\nabla \eta\|_{\mathcal{H}^{m-2}}\right) d \tau .
\end{aligned}
$$

Second, we deal with the commutator term $\mathcal{C}_{4}$. Note that

$$
\mathcal{C}_{4}=-\sum_{|\beta| \geq 1, \beta+\gamma=\alpha} \sum_{i=1}^{2} D_{\beta, \gamma} \mathcal{Z}^{\beta} u_{i} \cdot \mathcal{Z}^{\gamma} \partial_{y^{i}} \eta
$$




$$
-\sum_{|\beta| \geq 1, \beta+\gamma=\alpha} D_{\beta, \gamma} \mathcal{Z}^{\beta}(u \cdot N) \mathcal{Z}^{\gamma} \partial_{z} \eta-u \cdot N \sum_{|\beta| \leq m-2} D_{\beta} \partial_{z} \mathcal{Z}^{\beta} \eta
$$

By using Lemma 2.2 we can easily obtain that

$$
\begin{gathered}
\sum_{|\beta| \geq 1, \beta+\gamma=\alpha} \sum_{i=1}^{2} D_{\beta, \gamma} \int_{0}^{t}\left\|\mathcal{Z}^{\beta} u_{i} \cdot \mathcal{Z}^{\gamma} \partial_{y^{i}} \eta\right\|^{2} d \tau \\
\leq D_{m+2} P(M(t)) \int_{0}^{t}\left(\left\|u^{\epsilon}\right\|_{\mathcal{H}^{m}}^{2}+\left\|\nabla u^{\epsilon}\right\|_{\mathcal{H}^{m-1}}^{2}\right) d \tau .
\end{gathered}
$$

Since we want to get an estimate independent of $\partial_{z} \eta^{\epsilon}$, by using Hardy's inequality, we have

$$
\begin{aligned}
\sum_{|\beta| \leq m-2} \int_{0}^{t}\left\|u \cdot N D_{\beta} \partial_{z} \mathcal{Z}^{\beta} \eta\right\|^{2} d \tau & \leq \sum_{|\beta| \leq m-2} \int_{0}^{t}\left\|\frac{u^{\epsilon} \cdot N}{\varphi(z)} D_{\beta} Z_{3} \mathcal{Z}^{\beta} \eta\right\|^{2} d \tau \\
& \leq D_{m+2} P(M(t)) \int_{0}^{t}\left(\|u\|_{\mathcal{H}^{m}}^{2}+\|\nabla u\|_{\mathcal{H}^{m-1}}^{2}\right) d \tau
\end{aligned}
$$

Also, we note that for $|\beta| \geq 1, \beta+\gamma=\alpha$ and $|\alpha|=m-1$, it holds

$$
\begin{aligned}
\mathcal{Z}^{\beta}(u \cdot N) \mathcal{Z}^{\gamma} \partial_{z} \eta & =\frac{1}{\varphi(z)} \mathcal{Z}^{\beta}(u \cdot N) \cdot \varphi(z) \mathcal{Z}^{\gamma} \partial_{z} \eta \\
& =\sum_{|\tilde{\beta}| \leq \beta,|\tilde{\gamma}| \leq \gamma} D_{\tilde{\beta}, \tilde{\gamma}} \mathcal{Z}^{\tilde{\beta}}\left(\frac{u^{\epsilon} \cdot N}{\varphi(z)}\right) \mathcal{Z}^{\tilde{\gamma}}\left(Z_{3} \eta\right),
\end{aligned}
$$

where $|\tilde{\beta}|+|\tilde{\gamma}| \leq m-1,|\tilde{\gamma}| \leq m-2$ and $D_{\tilde{\beta}, \tilde{\gamma}}$ are some smooth bounded coefficient. By using Hardy's inequality, we have

$$
\begin{gathered}
\sum_{|\beta| \geq 1, \beta+\gamma=\alpha} \int_{0}^{t}\left\|D_{\beta, \gamma} \mathcal{Z}^{\beta}(u \cdot N) \mathcal{Z}^{\gamma} \partial_{z} \eta\right\|^{2} d \tau \\
\leq D_{m+2} P(M(t)) \int_{0}^{t}\left(\|u\|_{\mathcal{H}^{m}}^{2}+\|\nabla u\|_{\mathcal{H}^{m-1}}^{2}\right) d \tau .
\end{gathered}
$$

Therefore, from (3.139)-(3.142), we get

$$
\int_{0}^{t}\left\|\mathcal{C}_{4}\right\|^{2} d \tau \leq D_{m+2} P(M(t)) \int_{0}^{t}\left(\|u\|_{\mathcal{H}^{m}}^{2}+\|\nabla u\|_{\mathcal{H}^{m-1}}^{2}\right) d \tau .
$$

Next, it remains to deal with the term involving the pressure $p$. As above, we use the split $p=p_{1}+p_{2}$ and integrate by parts the term involving $p_{2}$. We have

$$
\begin{aligned}
& \left|\int_{0}^{t} \int_{\Omega} \mathcal{Z}^{\alpha}\left(\chi \Pi\left(\nabla^{2} p \nu\right)\right) \cdot \mathcal{Z}^{\alpha} \eta d x d \tau\right| \\
& \leq D_{m+2} \int_{0}^{t}\left(\left\|\nabla^{2} p_{1}\right\|_{\mathcal{H}^{m-1}}\|\eta\|_{\mathcal{H}^{m-1}}+\left\|\nabla p_{2}\right\|_{\mathcal{H}^{m-1}}\left(\|\nabla \eta\|_{\mathcal{H}^{m-1}}+\|\eta\|_{\mathcal{H}^{m-1}}\right)\right) d \tau .
\end{aligned}
$$


Finally, from (3.124)-(3.126) and (3.130), we get

$$
\begin{aligned}
\int_{0}^{t}\|F\|_{\mathcal{H}^{m-1}}^{2} d \tau \leq & D_{m+2}(1+P(M(t))) \int_{0}^{t}\left(\|u\|_{\mathcal{H}^{m}}^{2}+\|\nabla u\|_{\mathcal{H}^{m-1}}^{2}\right) d \tau \\
& +D \epsilon^{2} \int_{0}^{t}\left(\|\nabla u\|_{\mathcal{H}^{m}}^{2}+\|\nabla \eta\|_{\mathcal{H}^{m-1}}^{2}\right) d \tau+D_{m+2} \int_{0}^{t}\|\nabla p\|_{\mathcal{H}^{m-1}}^{2} d \tau \\
& +D \int_{0}^{t}\|\nabla(n \nabla \phi)\|_{\mathcal{H}^{m-1}}^{2} d \tau .
\end{aligned}
$$

By collecting (3.134), (3.135), (3.137), (3.143)-(3.145), Young's inequality, and the inductive assumption, we can get (3.121). Hence, the proof of Lemma 3.10 is completed.

3.5. Pressure Estimates. To enclose our a priori estimates, it remains to estimate the pressure terms and the $L^{\infty}$ norms. The aim of this subsection is to give the pressure estimates and present the $L^{\infty}$ estimates in next subsection.

Lemma 3.11. For every $m \geq 2$, we have the following estimates:

$$
\begin{aligned}
\int_{0}^{t}\left(\left\|\nabla p_{1}\right\|_{\mathcal{H}^{m-1}}^{2}+\left\|\nabla^{2} p_{1}\right\|_{\mathcal{H}^{m-1}}^{2}\right) d \tau \leq & D_{m+2} P(M(t)) \int_{0}^{t}\left(\|u\|_{\mathcal{H}^{m}}^{2}+\|\nabla u\|_{\mathcal{H}^{m-1}}^{2}\right) d \tau \\
& +\int_{0}^{t}\left(\|n \nabla \phi\|_{\mathcal{H}^{m}}^{2}+\|\nabla(n \nabla \phi)\|_{\mathcal{H}^{m-1}}^{2}\right) d \tau \\
\int_{0}^{t}\left(\left\|p_{2}\right\|_{\mathcal{H}^{m-1}}^{2}+\left\|\nabla p_{2}\right\|_{\mathcal{H}^{m-1}}^{2}\right) d \tau \leq & D_{m+2} \epsilon \int_{0}^{t}\left(\|u\|_{\mathcal{H}^{m}}^{2}+\|\nabla u\|_{\mathcal{H}^{m-1}}^{2}\right) d \tau .
\end{aligned}
$$

Proof. From (3.13) and (3.14), we obtain that

$$
\left\{\begin{array}{l}
\Delta\left(\partial_{t}^{\alpha_{0}} p_{1}\right)=-\partial_{t}^{\alpha_{0}} \nabla \cdot(u \cdot \nabla u)-\partial_{t}^{\alpha_{0}} \nabla \cdot(n \nabla \phi) \quad \text { in } \quad \Omega, \\
\partial_{\nu}\left(\partial_{t}^{\alpha_{0}} p_{1}\right)=-\partial_{t}^{\alpha_{0}}(u \cdot \nabla u) \cdot \nu-\partial_{t}^{\alpha_{0}}(n \nabla \phi) \cdot \nu \quad \text { on } \quad \partial \Omega
\end{array}\right.
$$

and

$$
\left\{\begin{array}{l}
\Delta\left(\partial_{t}^{\alpha_{0}} p_{2}\right)=0 \quad \text { in } \quad \Omega, \\
\partial_{\nu}\left(\partial_{t}^{\alpha_{0}} p_{2}\right)=\epsilon \partial_{t}^{\alpha_{0}} \Delta u \cdot \nu \text { on } \partial \Omega .
\end{array}\right.
$$

First, we deal with $p_{1}$. From the standard elliptic regularity results with Neumann boundary condition, we obtain that

$$
\begin{aligned}
&\left\|\nabla \partial_{t}^{\alpha_{0}} p_{1}^{\epsilon}\right\|_{H_{c o}^{\left|\alpha_{1}\right|}}+\left\|\nabla^{2} \partial_{t}^{\alpha_{0}} p_{1}^{\epsilon}\right\|_{H_{c o}^{\left|\alpha_{1}\right|}} \\
& \leq D_{m+1}\left(\left\|\partial_{t}^{\alpha_{0}} \nabla \cdot(u \cdot \nabla u+n \nabla \phi)\right\|_{H_{c o}^{\left|\alpha_{1}\right|}}+\left\|\partial_{t}^{\alpha_{0}}(u \cdot \nabla u+n \nabla \phi)\right\|\right. \\
&\left.\quad+\left|\partial_{t}^{\alpha_{0}}(u \cdot \nabla u+n \nabla \phi) \cdot \nu\right|_{H^{m-\left|\alpha_{0}\right|-\frac{1}{2}}(\partial \Omega)}\right),
\end{aligned}
$$

where $\left|\alpha_{0}\right|+\left|\alpha_{1}\right|=m-1$. Due to $u \cdot \nu=0$ on $\partial \Omega$ and Lemma 2.3, we get

$$
\begin{aligned}
& \left|\partial_{t}^{\alpha_{0}}(u \cdot \nabla u+n \nabla \phi) \cdot \nu\right|_{H^{m-\left|\alpha_{0}\right|-\frac{1}{2}}(\partial \Omega)} \\
\leq & D_{m+2}\left(\|\nabla(u \otimes u)\|_{\mathcal{H}^{m-1}}+\|u \otimes u\|_{\mathcal{H}^{m}}+\|\nabla(n \nabla \phi)\|_{\mathcal{H}^{m-1}}+\|n \nabla \phi\|_{\mathcal{H}^{m}}\right) .
\end{aligned}
$$


Using Lemma 2.2, we easily get (3.146).

It remains to estimate $p_{2}$. By using the standard elliptic regularity results with Neumann boundary condition again, we obtain that

$$
\left\|\partial_{t}^{\alpha_{0}} p_{2}\right\|_{H_{c o}^{m-\left|\alpha_{0}\right|-1}}+\left\|\nabla \partial_{t}^{\alpha_{0}} p_{2}\right\|_{H_{c o}^{m-\left|\alpha_{0}\right|-1}} \leq D_{m} \epsilon\left|\partial_{t}^{\alpha_{0}} \Delta u^{\epsilon} \cdot \nu\right|_{H^{m-\left|\alpha_{0}\right|-\frac{3}{2}}(\partial \Omega)} .
$$

Since

$$
\partial_{t}^{\alpha_{0}} \Delta u \cdot \nu=2\left(\partial_{t}^{\alpha_{0}} \nabla \cdot(S u \nu)-\sum_{j} \partial_{t}^{\alpha_{0}}\left(S u \partial_{j} \nu\right)_{j}\right)
$$

we have

$$
\begin{aligned}
\left|\partial_{t}^{\alpha_{0}} \Delta u \cdot \nu\right|_{H^{m-\left|\alpha_{0}\right|-\frac{3}{2}}(\partial \Omega)} \leq & D\left|\partial_{t}^{\alpha_{0}} \nabla \cdot(S u \nu)\right|_{H^{m-\left|\alpha_{0}\right|-\frac{3}{2}}(\partial \Omega)} \\
& +D_{m+1}\left|\partial_{t}^{\alpha_{0}} \nabla u\right|_{H^{m-\left|\alpha_{0}\right|-\frac{3}{2}}(\partial \Omega)}
\end{aligned}
$$

Due to (3.23), we can further arrive at

$$
\begin{aligned}
\left|\partial_{t}^{\alpha_{0}} \Delta u \cdot \nu\right|_{H^{m-\left|\alpha_{0}\right|-\frac{3}{2}}(\partial \Omega)} \leq & D\left|\partial_{t}^{\alpha_{0}} \nabla \cdot(S u \nu)\right|_{H^{m-\left|\alpha_{0}\right|-\frac{3}{2}}(\partial \Omega)} \\
& +D_{m+1}\left|\partial_{t}^{\alpha_{0}} u\right|_{H^{m-\left|\alpha_{0}\right|-\frac{1}{2}}(\partial \Omega)}
\end{aligned}
$$

Let us estimate $\left|\partial_{t}^{\alpha_{0}} \nabla \cdot(S u \nu)\right|_{H^{m-\left|\alpha_{0}\right|-\frac{3}{2}}(\partial \Omega)}$. Thank to (3.21), we get

$$
\begin{aligned}
& \left|\partial_{t}^{\alpha_{0}} \nabla \cdot(S u \nu)\right|_{H^{m-\left|\alpha_{0}\right|-\frac{3}{2}}(\partial \Omega)} \\
\leq & D\left|\partial_{\nu} \partial_{t}^{\alpha_{0}}(S u \nu) \cdot \nu\right|_{H^{m-\left|\alpha_{0}\right|-\frac{3}{2}}(\partial \Omega)}+D\left(\left|\Pi \partial_{t}^{\alpha_{0}}(S u \nu)\right|_{H^{m-\left|\alpha_{0}\right|-\frac{1}{2}}(\partial \Omega)}\right. \\
& \left.+\left|\nabla \partial_{t}^{\alpha_{0}} u\right|_{H^{m-\left|\alpha_{0}\right|-\frac{3}{2}}(\partial \Omega)}\right) .
\end{aligned}
$$

Also, due to (3.23) and the Navier boundary condition (1.11), we get

$$
\begin{aligned}
\left|\partial_{t}^{\alpha_{0}} \nabla \cdot(S u \nu)\right|_{H^{m-\left|\alpha_{0}\right|-\frac{3}{2}}(\partial \Omega)} \leq & D\left|\partial_{\nu} \partial_{t}^{\alpha_{0}}(S u \nu) \cdot \nu\right|_{H^{m-\left|\alpha_{0}\right|-\frac{3}{2}}(\partial \Omega)} \\
& +\left|\partial_{t}^{\alpha_{0}} u\right|_{H^{m-\left|\alpha_{0}\right|-\frac{1}{2}}(\partial \Omega)}
\end{aligned}
$$

The first term in the right-hand side of (3.150) can be estimated as

$$
\begin{aligned}
& \left|\partial_{\nu} \partial_{t}^{\alpha_{0}}(S u \nu) \cdot \nu\right|_{H^{m-\left|\alpha_{0}\right|-\frac{3}{2}}(\partial \Omega)} \\
\leq & D\left|\partial_{\nu} \partial_{t}^{\alpha_{0}}\left(\partial_{\nu} u \cdot \nu\right)\right|_{H^{m-\left|\alpha_{0}\right|-\frac{3}{2}}(\partial \Omega)}+D_{m+1}\left|\nabla \partial_{t}^{\alpha_{0}} u\right|_{H^{m-\left|\alpha_{0}\right|-\frac{3}{2}}(\partial \Omega)} \\
\leq & D\left|\partial_{\nu} \partial_{t}^{\alpha_{0}}\left(\partial_{\nu} u \cdot \nu\right)\right|_{H^{m-\left|\alpha_{0}\right|-\frac{3}{2}}(\partial \Omega)}+D_{m+1}\left|\partial_{t}^{\alpha_{0}} u\right|_{H^{m-\left|\alpha_{0}\right|-\frac{1}{2}}(\partial \Omega)}
\end{aligned}
$$

By taking the normal derivative of (3.21) and using (1.24), we obtain that

$$
\begin{aligned}
& \left|\partial_{\nu} \partial_{t}^{\alpha_{0}}\left(\partial_{\nu} u \cdot \nu\right)\right|_{H^{m-\left|\alpha_{0}\right|-\frac{3}{2}}(\partial \Omega)} \\
\leq & D\left|\Pi \partial_{t}^{\alpha_{0}} \partial_{\nu} u\right|_{H^{m-\left|\alpha_{0}\right|-\frac{1}{2}}(\partial \Omega)}+D_{m+1}\left|\nabla \partial_{t}^{\alpha_{0}} u\right|_{H^{m-\left|\alpha_{0}\right|-\frac{3}{2}}(\partial \Omega)} \\
\leq & D_{m+2}\left|\partial_{t}^{\alpha_{0}} u\right|_{H^{m-\left|\alpha_{0}\right|-\frac{1}{2}}(\partial \Omega)}
\end{aligned}
$$

Consequently, we have

$$
\left|\partial_{t}^{\alpha_{0}} \Delta u \cdot \nu\right|_{H^{m-\left|\alpha_{0}\right|-\frac{3}{2}}(\partial \Omega)} \leq D_{m+2}\left|\partial_{t}^{\alpha_{0}} u\right|_{H^{m-\left|\alpha_{0}\right|-\frac{1}{2}}(\partial \Omega)} .
$$


By virtue of Lemma 2.3, we finally get (3.147). Therefore, we complete the proof of Lemma 3.11.

Substituting (3.121) and (3.146)-(3.147) into (3.118), we can obtain that

$$
\begin{aligned}
& \sup _{0 \leq \tau \leq t}\left(\|(n, c, u)\|_{\mathcal{H}^{m}}^{2}+\|\nabla(n, u)\|_{\mathcal{H}^{m-1}}^{2}+\|\nabla c\|_{\mathcal{H}^{m}}^{2}\right. \\
& \left.+\|\Delta(n, c)\|_{\mathcal{H}^{m-1}}^{2}\right)+\epsilon \int_{0}^{t}\left(\|\nabla u\|_{\mathcal{H}^{m}}^{2}+\left\|\nabla^{2} u\right\|_{\mathcal{H}^{m-1}}^{2}\right) d \tau \\
& +\int_{0}^{t}\left(\|\nabla n\|_{\mathcal{H}^{m}}^{2}+\|\Delta c\|_{\mathcal{H}^{m}}^{2}+\|\nabla \Delta(n, c)\|_{\mathcal{H}^{m-1}}^{2}\right) d \tau \\
& \leq D_{m+2}\left\{N_{m}(0)+(1+P(M(t))) \int_{0}^{t} N_{m}(\tau) d \tau\right. \\
& \left.+\int_{0}^{t}\left(\|n \nabla \phi\|_{\mathcal{H}^{m}}^{2}+\|\nabla(n \nabla \phi)\|_{\mathcal{H}^{m-1}}^{2}\right) d \tau\right\} .
\end{aligned}
$$

3.6. $L^{\infty}$ estimates. In order to close the estimate (3.151), we need to give the $L^{\infty}$ estimates for $(n, c, u)$.

Lemma 3.12. For every $m \geq 4$, we have the following estimates:

$$
\begin{aligned}
\|n\|_{W^{2, \infty}}^{2} & \leq D\left(N_{m}(t)+N_{m}^{2}(t)+N_{m}^{3}(t)\right) \\
\|u\|_{\mathcal{H}^{2, \infty}}^{2} & \leq D N_{m}(t) \\
\|c\|_{W^{2, \infty}}^{2} & \leq D\left(N_{m}(t)+N_{m}^{2}(t)\right) \\
\|\nabla c\|_{\mathcal{H}^{1, \infty}}^{2} & \leq D N_{m}(t) \\
\|\nabla \Delta c\|_{L^{\infty}}^{2} & \leq D\left(N_{m}(t)+N_{m}^{2}(t)\right) .
\end{aligned}
$$

Proof. In view of Lemma 2.3 and (3.68), we obtain that

$$
\|n\|_{W^{1, \infty}}^{2}+\|\nabla n\|_{\mathcal{H}^{1, \infty}}^{2}+\|u\|_{\mathcal{H}^{2, \infty}}^{2}+\|\nabla c\|_{\mathcal{H}^{1, \infty}}^{2}+\|c\|_{\mathcal{H}^{1, \infty}}^{2} \leq D N_{m}(t) .
$$

Hence, we get (3.153) and (3.155). Also, by virtue of Lemma 2.3 and (3.68), we have

$$
\begin{aligned}
\left\|\nabla^{2} c\right\|_{L^{\infty}}^{2} & \leq D\left(\|\Delta c\|_{L^{\infty}}^{2}+\|\nabla c\|_{H_{c o}^{1, \infty}}^{2}\right) \\
& \leq D\left(\|\nabla \Delta c\|_{H_{c o}^{1}}^{2}+\|\Delta c\|_{H_{c o}^{2}}^{2}+\|\nabla c\|_{H_{c o}^{3}}^{2}\right) .
\end{aligned}
$$

Due to the equations (1.6) and (3.157), we deduce that

$$
\|\nabla \Delta c\|_{H_{c o}^{1}}^{2} \leq D\left(N_{m}(t)+N_{m}^{2}(t)\right) \text {. }
$$

The combination of (3.157)-(3.159) yields (3.154). Based on the equation (1.6), (3.154) and (3.157), we can easily get

$$
\|\nabla \Delta c\|_{L^{\infty}}^{2} \leq D\left\|\nabla\left(c_{t}+u \cdot \nabla c+n c\right)\right\|_{L^{\infty}}^{2} \leq D\left(N_{m}(t)+N_{m}^{2}(t)\right),
$$

which gives (3.156). Finally, similar to (3.158), we have

$$
\left\|\nabla^{2} n\right\|_{L^{\infty}}^{2} \leq D\left(\|\nabla \Delta n\|_{H_{c o}^{1}}^{2}+\|\Delta n\|_{H_{c o}^{2}}^{2}+\|\nabla n\|_{H_{c o}^{3}}^{2}\right) .
$$


By virtue of the equation (1.5) and (3.153)-(3.157), we obtain that

$$
\|\nabla \Delta n\|_{H_{c o}^{1}}^{2} \leq D\left(N_{m}(t)+N_{m}^{2}(t)+N_{m}(t)\|\nabla \Delta c\|_{H_{c o}^{1}}^{2}\right) .
$$

Furthermore, with the help of the equation (1.6), we arrive at

$$
\begin{aligned}
\|\nabla \Delta c\|_{H_{c o}^{1}}^{2} & \leq D\left(\|\nabla c\|_{\mathcal{H}^{2}}^{2}+\|\nabla(u \cdot \nabla c)\|_{H_{c o}^{1}}^{2}+\|\nabla(n c)\|_{H_{c o}^{1}}^{2}\right) \\
& \leq D\left(N_{m}(t)+N_{m}^{2}(t)\right) .
\end{aligned}
$$

From (3.157) and (3.160)-(3.162), we get (3.152).

Finally, we prove the estimate for $\|\nabla u\|_{\mathcal{H}^{1, \infty}}$.

Lemma 3.13. For $m \geq 6$, we have the following estimate:

$$
\begin{aligned}
\|\nabla u\|_{\mathcal{H}^{1, \infty}}^{2} \leq & D\left(N_{m}(0)+N_{m}(t)+\left(1+P\left(N_{m}(t)\right)\right)\right. \\
& \left.\times \int_{0}^{t} P\left(N_{m}(\tau)\right) d \tau\right)+\delta \epsilon \int_{0}^{t}\left\|\nabla^{2} u\right\|_{\mathcal{H}^{4}}^{2} d \tau,
\end{aligned}
$$

where $\delta$ is a small enough constant.

Proof. We observe that, away from the boundary, the following estimate holds:

$$
\left\|\beta_{i} \nabla u^{\epsilon}\right\|_{L^{\infty}}^{2}+\left\|\beta_{i} \mathcal{Z} \nabla u^{\epsilon}\right\|_{L^{\infty}}^{2} \leq D\left\|u^{\epsilon}\right\|_{\mathcal{H}^{m}}, \quad m \geq 4,
$$

where $\left\{\beta_{i}\right\}$ is a partition of unity subordinated to the covering (1.21). In order to estimate the near boundary parts, we adopt the ideas in the Proposition 21 of [18]. Here, we use a local parametrization in the vicinity of the boundary given by a normal geodesic system:

$$
\Psi^{\nu}(y, z)=\left(\begin{array}{c}
y \\
\psi(y)
\end{array}\right)-z \nu(y),
$$

where

$$
\nu(y)=\frac{1}{\sqrt{1+|\nabla \psi(y)|^{2}}}\left(\begin{array}{c}
\partial_{1} \psi(y) \\
\partial_{2} \psi(y) \\
-1
\end{array}\right) .
$$

Now, we can extend $\nu$ and $\Pi$ in the interior by setting

$$
\nu\left(\Psi^{\nu}(y, z)\right)=\nu(y), \quad \Pi\left(\Psi^{\nu}(y, z)\right)=\Pi(y) .
$$

We observe $\partial_{z}=\partial_{\nu}$ and

$$
\left.\left.\left(\partial_{y^{i}}\right)\right|_{\Psi^{\nu}(y, z)} \cdot\left(\partial_{z}\right)\right|_{\Psi^{\nu}(y, z)}=0 .
$$

Hence, the Riemann metric $g$ has the following form

$$
g(y, z)=\left(\begin{array}{cc}
\widetilde{g}(y, z) & 0 \\
0 & 1
\end{array}\right) .
$$


Consequently, the Laplacian in this coordinate system reads:

$$
\Delta f=\partial_{z z} f+\frac{1}{2} \partial_{z}(\ln |g|) \partial_{z} f+\Delta_{\widetilde{g}} f
$$

where $|g|$ is the determinant of the matrix $g$ and $\Delta_{\widetilde{g}}$ is defined by

$$
\Delta_{\widetilde{g}} f=\frac{1}{|\widetilde{g}|^{\frac{1}{2}}} \sum_{1 \leq i, j \leq 2} \partial_{y^{i}}\left(\widetilde{g}^{i j}|\widetilde{g}|^{\frac{1}{2}} \partial_{y^{j}} f\right) .
$$

Here, $\left\{\widetilde{g}^{i j}\right\}$ is the inverse matrix to $g$ and (3.164) only involves the tangential derivatives.

With these preparation, we now turn to estimate the near boundary parts. By using Lemma 3.16 and (3.21), we have

$$
\|\chi \nabla u\|_{\mathcal{H}^{1, \infty}} \leq D_{3}\left(\left\|\chi \Pi \partial_{\nu} u\right\|_{\mathcal{H}^{1, \infty}}+\|u\|_{\mathcal{H}^{m}}+\|\nabla u\|_{\mathcal{H}^{m-1}}\right) .
$$

Hence, we need to estimate $\left\|\chi \Pi \partial_{\nu} u\right\|_{\mathcal{H}^{1, \infty}}$. To this end, we first introduce the vorticity

$$
\omega=\nabla \times u
$$

We find that

$$
\begin{aligned}
\Pi(\omega \times \nu) & =\Pi\left(\nabla u-(\nabla u)^{t}\right) \nu \\
& =\Pi\left(\partial_{\nu} u-\nabla(u \cdot \nu)+(\nabla \nu)^{t} u+u \times(\nabla \times \nu)\right) .
\end{aligned}
$$

Consequently, we have

$$
\left\|\chi \Pi \partial_{\nu} u\right\|_{\mathcal{H}^{1, \infty}} \leq D_{3}\left(\|\chi \Pi(\omega \times \nu)\|_{\mathcal{H}^{1, \infty}}+\|u\|_{\mathcal{H}^{2, \infty}}\right) .
$$

By using (3.165) again, we get

$$
\|\chi \nabla u\|_{\mathcal{H}^{1, \infty}} \leq D_{3}\left(\|\chi \Pi(\omega \times \nu)\|_{\mathcal{H}^{1, \infty}}+\|u\|_{\mathcal{H}^{m}}+\|\nabla u\|_{\mathcal{H}^{m-1}}\right) .
$$

In order to conclude the estimate (3.163), we only need to estimate $\| \chi \Pi(\omega \times$ $\nu) \|_{\mathcal{H}^{1, \infty}}$. By setting in the support of $\chi$

$$
\widetilde{\omega}(y, z):=\omega^{\epsilon}\left(\Psi^{\nu}(y, z)\right), \quad \widetilde{u}(y, z):=u\left(\Psi^{\nu}(y, z)\right),
$$

we have

$$
\begin{aligned}
\widetilde{\omega}_{t}+(\widetilde{u})^{1} \partial_{y^{1}} \widetilde{\omega}+(\widetilde{u})^{2} \partial_{y^{2}} \widetilde{\omega}+\widetilde{u} \cdot n \partial_{z} \widetilde{\omega}= & \epsilon\left(\partial_{z z} \widetilde{\omega}+\frac{1}{2} \partial_{z}(\ln |g|) \partial_{z} \widetilde{\omega}+\Delta_{\widetilde{g}} \widetilde{\omega}\right)+\widetilde{F}_{1}, \\
\widetilde{u}_{t}+(\widetilde{u})^{1} \partial_{y^{1}} \widetilde{u}+(\widetilde{u})^{2} \partial_{y^{2}} \widetilde{u}+\widetilde{u} \cdot n \partial_{z} \widetilde{u}= & \epsilon\left(\partial_{z z} \widetilde{u}+\frac{1}{2} \partial_{z}(\ln |g|) \partial_{z} \widetilde{u}+\Delta_{\widetilde{g}} \widetilde{u}\right) \\
& -(\nabla p) \circ \Psi^{\nu}-(n \nabla \phi) \circ \Psi^{\nu}
\end{aligned}
$$

where

$$
\widetilde{F}_{1}:=F_{1}\left(\Psi^{\nu}(y, z)\right), \quad F_{1}:=(\omega \cdot \nabla) u-\nabla n \times \nabla \phi .
$$

By using (1.24) and (3.166) on the boundary, we have

$$
\Pi(\widetilde{\omega} \times \nu)=2 \Pi\left((\nabla \nu)^{t} \widetilde{u}-\zeta \widetilde{u}\right) .
$$


Consequently, we introduce the following quantity:

$$
\widetilde{\eta}(y, z):=\chi \Pi\left(\widetilde{\omega} \times \nu-2(\nabla \nu)^{t} \widetilde{u}+2 \zeta \widetilde{u}\right) .
$$

We thus get that $\widetilde{\eta}(y, 0)=0$ and that $\widetilde{\eta}$ solves the equation

$$
\begin{aligned}
\widetilde{\eta}_{t}+(\widetilde{u})^{1} \partial_{y^{1}} \widetilde{\eta}+(\widetilde{u})^{2} \partial_{y^{2}} \widetilde{\eta}+\widetilde{u} \cdot \nu \partial_{z} \widetilde{\eta}= & \epsilon\left(\partial_{z z} \widetilde{\eta}+\frac{1}{2} \partial_{z}(\ln |g|) \partial_{z} \widetilde{\eta}\right) \\
& +\chi \Pi\left(\widetilde{F}_{1} \times \nu\right)+\widetilde{F}^{u}+\widetilde{F}^{\chi}+\widetilde{F}^{\kappa},
\end{aligned}
$$

where

$$
\begin{aligned}
\widetilde{F}^{u}= & 2 \chi \Pi\left((\nabla \nu)^{t}(\nabla p+n \nabla \phi)-\zeta \nabla p-\zeta(n \nabla \phi)\right) \circ \Psi^{\nu}, \\
\widetilde{F}^{\chi}= & \left(\left((\widetilde{u})^{1} \partial_{y^{1}}+(\widetilde{u})^{2} \partial_{y^{2}}+\widetilde{u} \cdot \nu \partial_{z}\right) \chi\right) \Pi\left(\widetilde{\omega} \times \nu-2(\nabla \nu)^{t} \widetilde{u}+2 \zeta \widetilde{u}\right) \\
& -\epsilon\left(\partial_{z z} \chi+2 \partial_{z} \chi \partial_{z}+\frac{1}{2} \partial_{z}(\ln |g|) \partial_{z} \chi\right) \Pi\left(\widetilde{\omega} \times \nu-2(\nabla \nu)^{t} \widetilde{u}+2 \zeta \widetilde{u}\right), \\
\widetilde{F}^{\kappa}= & \chi\left(\left((\widetilde{u})^{1} \partial_{y^{1}}+(\widetilde{u})^{2} \partial_{y^{2}}\right) \Pi\right)\left(\widetilde{\omega} \times \nu-2(\nabla \nu)^{t} \widetilde{u}+2 \zeta \widetilde{u}\right)+\epsilon \chi \Pi\left(\Delta_{\widetilde{g}} \widetilde{\omega} \times \nu\right) \\
& -2 \epsilon \chi \Pi\left((\nabla \nu)^{t} \Delta_{\widetilde{g}} \widetilde{u}\right)-2 \chi \Pi\left(\left(\left((\widetilde{u})^{1} \partial_{y^{1}}+(\widetilde{u})^{2} \partial_{y^{2}}\right)(\nabla \nu)^{t}\right) \widetilde{u}\right) \\
& +\chi \Pi\left(\widetilde{\omega} \times\left((\widetilde{u})^{1} \partial_{y^{1}}+(\widetilde{u})^{2} \partial_{y^{2}}\right) \nu\right)-2 \zeta \epsilon \chi \Pi\left(\Delta_{\widetilde{g}} \widetilde{u}\right) .
\end{aligned}
$$

We know that both $\Pi$ and $\nu$ do not dependent the normal variable. Due to $\Delta_{\widetilde{g}}$ only involving the tangential derivatives and the derivatives of $\chi$ compactly supported away from the boundary, we easily obtain that

$$
\begin{aligned}
&\left\|\chi \Pi\left(\widetilde{F}_{1} \times \nu\right)\right\|_{\mathcal{H}^{1, \infty}} \leq D\left(\nabla u\left\|_{\mathcal{H}^{1, \infty}}^{2}+\right\| \nabla n \times \nabla \phi \|_{\mathcal{H}^{1, \infty}}\right), \\
&\left\|\widetilde{F}^{u}\right\|_{\mathcal{H}^{1, \infty}} \leq D_{3}\left(\|\Pi \nabla p\|_{\mathcal{H}^{1, \infty}}+\|\Pi(n \nabla \phi)\|_{\mathcal{H}^{1, \infty}}\right), \\
&\left\|\widetilde{F}^{\chi}\right\|_{\mathcal{H}^{1, \infty}} \leq D_{3}\left(\|u\|_{\mathcal{H}^{1, \infty}}\|u\|_{\mathcal{H}^{2, \infty}}+\epsilon\|u\|_{\mathcal{H}^{3, \infty}}\right), \\
&\left\|\widetilde{F}^{\kappa}\right\|_{\mathcal{H}^{1, \infty}} \leq D_{4}\left(\|u\|_{\mathcal{H}^{1, \infty}}^{2}+\|\nabla u\|_{\mathcal{H}^{1, \infty}}\|u\|_{\mathcal{H}^{1, \infty}}\right. \\
&\left.+\epsilon\|u\|_{\mathcal{H}^{3, \infty}}+\epsilon\|\nabla u\|_{\mathcal{H}^{3, \infty}}\right) .
\end{aligned}
$$

Therefore, by using Lemmas 2.3 and 3.12 , we get that

$$
\|\widetilde{F}\|_{\mathcal{H}^{1, \infty}}^{2} \leq D_{4}\left(\|\Pi \nabla p\|_{\mathcal{H}^{1, \infty}}^{2}+\epsilon^{2}\|\nabla u\|_{\mathcal{H}^{3, \infty}}^{2}+N_{m}(t)+N_{m}(t)^{2}\right),
$$

where $\widetilde{F}:=\chi \Pi\left(\widetilde{F}_{1} \times \nu\right)+\widetilde{F}^{u}+\widetilde{F}^{\chi}+\widetilde{F}^{\kappa}$. A crucial estimate towards the proof of Lemma 3.13 is the following:

Lemma 3.14 ([18]). Consider $\rho$ a smooth solution of

$$
\rho_{t}+u \cdot \nabla \rho=\epsilon \partial_{z z} \rho+\mathcal{S}, \quad z>0, \quad \rho(t, y, 0)=0
$$

for some smooth divergence free vector field $u$ such that $u \cdot \nu$ vanishes on the boundary. Assume that $\rho$ and $\mathcal{S}$ are compactly supported in $z$. Then, we have the estimate

$$
\begin{aligned}
\|\rho\|_{\mathcal{H}^{1, \infty}} \leq & D\|\rho(0)\|_{\mathcal{H}^{1, \infty}}+D \int_{0}^{t}\left\{\left(\|u\|_{\mathcal{H}^{2, \infty}}+\left\|\partial_{z} u\right\|_{\mathcal{H}^{1, \infty}}\right)\right. \\
& \left.\times\left(\|\rho\|_{\mathcal{H}^{1, \infty}}+\|\rho\|_{\mathcal{H}^{m_{0}+3}}\right)+\|\mathcal{S}\|_{\mathcal{H}^{1, \infty}}\right\} d \tau
\end{aligned}
$$


for $m_{0} \geq 2$.

In order to use Lemma 3.14 , we shall eliminate $\partial_{z}(\ln |g|) \partial_{z} \widetilde{\eta}$ in (3.170). We set

$$
\widetilde{\eta}:=\frac{1}{|g|^{\frac{1}{4}}} \bar{\eta}=\bar{\gamma} \bar{\eta}
$$

We note that

$$
\|\widetilde{\eta}\|_{\mathcal{H}^{1, \infty}} \sim\|\bar{\eta}\|_{\mathcal{H}^{1, \infty}}
$$

and $\bar{\eta}$ solve the equations

$$
\bar{\eta}_{t}+(\widetilde{u})^{1} \partial_{y^{1}} \bar{\eta}+(\widetilde{u})^{2} \partial_{y^{2}} \bar{\eta}+(u \cdot n) \partial_{z} \bar{\eta}-\epsilon \partial_{z z} \bar{\eta}=\mathcal{S},
$$

where

$$
\mathcal{S}:=\frac{1}{\bar{\gamma}}\left(\chi \Pi\left(\widetilde{F}_{1} \times \nu\right)+\widetilde{F}^{u}+\widetilde{F}^{\chi}+\widetilde{F}^{\kappa}+\epsilon \partial_{z z} \bar{\gamma} \bar{\eta}+\frac{\epsilon}{2} \partial_{z} \ln |g| \partial_{z} \bar{\gamma} \bar{\eta}-(\widetilde{u} \cdot \nabla \bar{\gamma}) \bar{\eta}\right) .
$$

Applying Lemma 3.14 to (3.177), we obtain that

$$
\begin{aligned}
\|\bar{\eta}\|_{\mathcal{H}^{1, \infty}} \leq D & \left\{\left\|\bar{\eta}_{0}\right\|_{\mathcal{H}^{1, \infty}}+\int_{0}^{t}\left(\left(\|u\|_{\mathcal{H}^{2, \infty}}+\left\|\partial_{z} u\right\|_{\mathcal{H}^{1, \infty}}\right)\right.\right. \\
& \left.\left.\times\left(\|\bar{\eta}\|_{\mathcal{H}^{1, \infty}}+\|\bar{\eta}\|_{\mathcal{H}^{m_{0}+3}}\right)+\|\mathcal{S}\|_{\mathcal{H}^{1, \infty}}\right) d \tau\right\} .
\end{aligned}
$$

It remains to estimate $\|S\|_{\mathcal{H}^{1, \infty}}$. Due to Lemmas 2.3, 3.15] and (3.175), we have

$$
\begin{aligned}
\int_{0}^{t}\|\mathcal{S}\|_{\mathcal{H}^{1, \infty}}^{2} d \tau & \leq D \int_{0}^{t}\left(\left\|\Pi \nabla p^{\epsilon}\right\|_{\mathcal{H}^{1, \infty}}^{2}+\epsilon^{2}\left\|\nabla u^{\epsilon}\right\|_{\mathcal{H}^{3, \infty}}^{2}+N_{m}(t)+N_{m}(t)^{2}\right) d \tau \\
& \leq D_{m+2}\left(1+P\left(N_{m}(t)\right)\right) \int_{0}^{t} P\left(N_{m}(\tau)\right) d \tau+\delta \epsilon \int_{0}^{t}\left\|\nabla^{2} u\right\|_{\mathcal{H}^{4}}^{2} d \tau
\end{aligned}
$$

By virtue of (3.180) and Lemma 3.17, we get

$$
\begin{aligned}
\|\bar{\eta}\|_{\mathcal{H}^{1, \infty}}^{2} \leq & D_{m+2}\left\{\left\|\bar{\eta}_{0}\right\|_{\mathcal{H}^{1, \infty}}^{2}+\left(1+P\left(N_{m}(t)\right)\right) \int_{0}^{t} P\left(N_{m}(\tau)\right) d \tau\right. \\
& \left.+\delta \epsilon \int_{0}^{t}\left\|\nabla^{2} u\right\|_{\mathcal{H}^{4}}^{2} d \tau\right\} .
\end{aligned}
$$

Finally, the combination of (3.165), (3.168), (3.169) and (3.181) yields (3.163). Therefore, we complete the proof of Lemma 3.14

3.7. Proof of Theorem 3.1. It suffices to combine (3.151), Lemma 3.12 and Lemma 3.13 . 


\section{Proof of Theorem 1.1}

In this section, we will show how to combine our a priori estimates to prove the uniform existence results. Let us fix $m \geq 6$ and consider the initial data satisfy

$$
\mathcal{I}_{m}(0)=\sup _{0<\epsilon \leq 1}\left\|\left(n_{0}^{\epsilon}, c_{0}^{\epsilon}, u_{0}^{\epsilon}\right)\right\|_{\mathcal{E}_{C N S}^{m, \epsilon}} \leq \widetilde{D}_{3}
$$

For such initial data, we are not aware of a local existence result for the problem (1.5)-(1.11), so we first need to prove the local existence results for (1.5)-(1.11) by using the energy estimates obtained in Section 3 and a classical iteration scheme. By virtue of the definition of $\mathcal{E}_{C N S}^{m, \epsilon}$, there exists a sequence of smooth approximate initial data $\left(n_{0}^{\epsilon, \delta}, c_{0}^{\epsilon, \delta}, u_{0}^{\epsilon, \delta}\right)$ ( $\delta$ being a regularization parameter) which has enough space regularity so that the time derivatives at initial data can be defined by the chemotaxis-Navier-Stokes system and the boundary compatibility conditions can be satisfied.

Fixed $\epsilon \in(0,1]$, we construct approximate solutions as follows:

(1) Define $\left(n^{0}, c^{0}, u^{0}\right)=\left(n_{0}^{\epsilon, \delta}, c_{0}^{\epsilon, \delta}, u_{0}^{\epsilon, \delta}\right)$.

(2) Assume that $\left(n^{k-1}, c^{k-1}, u^{k-1}\right)$ has been defined for $k \geq 1$. Let $\left(n^{k}, c^{k}, u^{k}\right)$ be the unique solution to the following linearized initial boundary value problem:

$$
\left\{\begin{array}{l}
n_{t}^{k}-\Delta n^{k}=-u^{k-1} \cdot \nabla n^{k}-\nabla \cdot\left(n^{k} \cdot \nabla c^{k-1}\right), \\
c_{t}^{k}-\Delta c^{k}=-u^{k-1} \cdot \nabla c^{k}-n^{k-1} c^{k} \\
u_{t}^{k}-\epsilon \Delta u^{k}+u^{k-1} \cdot \nabla u^{k}+\nabla p^{k}=n^{k} \nabla \phi \\
\operatorname{div} u^{k}=0 \\
\left.\left(n^{k}, c^{k}, u^{k}\right)\right|_{t=0}=\left(n_{0}^{\epsilon, \delta}, c_{0}^{\epsilon, \delta}, u_{0}^{\epsilon, \delta}\right) .
\end{array}\right.
$$

in $(0, T) \times \Omega$ with the boundary conditions

$$
\frac{\partial n^{k}}{\partial \nu}=\frac{\partial c^{k}}{\partial \nu}=0, \quad u^{k} \cdot \nu=0, \quad\left(S u^{k} \cdot \nu\right)_{\tau}=-\zeta u_{\tau}^{k} .
$$

Since $n^{k}, c^{k}$ and $u^{k}$ are decoupled, the existence of the global smooth solution $\left(n^{k}, c^{k}, u^{k}\right)$ of (4.2) and (4.3) can be obtained by using the classical methods, for example, see [7,25, 31].

By using the a priori estimates given in Theorem 3.1 and an induction argument, we obtain that there exist a uniform time $\widetilde{T}_{1}$ and constant $\widetilde{D}_{4}$ (independent of $\epsilon$ and $\delta$ ) such that it holds for $\left(n^{k}, c^{k}, u^{k}\right), k \geq 1$ that

$$
\begin{aligned}
& \sup _{0 \leq \tau \leq t}\left\{\left\|\left(n^{k}, c^{k}, u^{k}\right)\right\|_{\mathcal{H}^{m}}^{2}+\left\|\nabla\left(n^{k}, u^{k}\right)\right\|_{\mathcal{H}^{m-1}}^{2}+\left\|\nabla c^{k}\right\|_{\mathcal{H}^{m}}^{2}\right. \\
& \left.+\left\|\Delta\left(n^{k}, c^{k}\right)\right\|_{\mathcal{H}^{m-1}}^{2}+\left\|\nabla u^{k}\right\|_{1, \infty}^{2}\right\}+\epsilon \int_{0}^{t}\left(\left\|\nabla u^{k}\right\|_{\mathcal{H}^{m}}^{2}+\left\|\nabla^{2} u^{k}\right\|_{\mathcal{H}^{m-1}}^{2}\right) d \tau \\
& +\int_{0}^{t}\left(\left\|\nabla n^{k}\right\|_{\mathcal{H}^{m}}^{2}+\left\|\Delta c^{k}\right\|_{\mathcal{H}^{m}}^{2}+\left\|\nabla \Delta\left(n^{k}, c^{k}\right)\right\|_{\mathcal{H}^{m-1}}^{2}\right) d \tau \leq \widetilde{D}_{4}, \quad \forall t \in\left[0, \widetilde{T}_{1}\right]
\end{aligned}
$$


where $\widetilde{T}_{1}$ and $\widetilde{D}_{4}$ depend only on $\mathcal{I}_{m}(0)$. In view of the above uniform estimates, there exists a uniform time $\widetilde{T}_{2}$ (independent $\epsilon$ and $\delta$ ) such that $\left(n^{k}, c^{k}, u^{k}\right)$ converges to a limit $\left(n^{\epsilon, \delta}, c^{\epsilon, \delta}, u^{\epsilon, \delta}\right)$ as $k \rightarrow+\infty$ in the following strong sense:

$$
\begin{array}{rll}
\left(n^{k}, c^{k}\right) \rightarrow\left(n^{\epsilon, \delta}, c^{\epsilon, \delta}\right) & \text { in } & L^{\infty}\left(0, \widetilde{T}_{2} ; H^{1}\right), \\
u^{k} \rightarrow u^{\epsilon, \delta} & \text { in } & L^{\infty}\left(0, \widetilde{T}_{2} ; L^{2}\right), \\
\nabla u^{k} \rightarrow \nabla u^{\epsilon, \delta} & \text { in } & L^{2}\left(0, \widetilde{T}_{2} ; L^{2}\right) .
\end{array}
$$

It is easy to deduce that $\left(n^{\epsilon, \delta}, c^{\epsilon, \delta}, u^{\epsilon, \delta}\right)$ is a weak solution to the system (1.5)-(1.11) with the initial data $\left(n_{0}^{\epsilon, \delta}, c_{0}^{\epsilon, \delta}, u_{0}^{\epsilon, \delta}\right)$. Furthermore, due to the lower semi-continuity of norms, we obtain that

$$
\begin{aligned}
& \sup _{0 \leq \tau \leq t}\left(\left\|\left(n^{\epsilon, \delta}, c^{\epsilon, \delta}, u^{\epsilon, \delta}\right)\right\|_{\mathcal{H}^{m}}^{2}+\left\|\nabla\left(n^{\epsilon, \delta}, u^{\epsilon, \delta}\right)\right\|_{\mathcal{H}^{m-1}}^{2}+\left\|\nabla c^{\epsilon, \delta}\right\|_{\mathcal{H}^{m}}^{2}\right. \\
& \left.+\left\|\Delta\left(n^{\epsilon, \delta}, c^{\epsilon, \delta}\right)\right\|_{\mathcal{H}^{m-1}}^{2}+\left\|\nabla u^{\epsilon, \delta}\right\|_{1, \infty}^{2}\right)+\epsilon \int_{0}^{t}\left(\left\|\nabla u^{\epsilon, \delta}\right\|_{\mathcal{H}^{m}}^{2}+\left\|\nabla^{2} u^{\epsilon, \delta}\right\|_{\mathcal{H}^{m-1}}^{2}\right) d \tau \\
& +\int_{0}^{t}\left(\left\|\nabla n^{\epsilon, \delta}\right\|_{\mathcal{H}^{m}}^{2}+\left\|\Delta c^{\epsilon, \delta}\right\|_{\mathcal{H}^{m}}^{2}+\left\|\nabla \Delta\left(n^{\epsilon, \delta}, c^{\epsilon, \delta}\right)\right\|_{\mathcal{H}^{m-1}}^{2}\right) d \tau \leq \widetilde{D}_{4}, \quad \forall t \in\left[0, \widetilde{T}_{2}\right] .
\end{aligned}
$$

Based on the uniform estimate (4.5) for $\left(n^{\epsilon, \delta}, c^{\epsilon, \delta}, u^{\epsilon, \delta}\right)$, we can pass the limit $\delta \rightarrow 0$ to get a strong solution $\left(n^{\epsilon}, c^{\epsilon}, u^{\epsilon}\right)$ of the system (1.5)-(1.11) with initial data $\left(n_{0}^{\epsilon}, c_{0}^{\epsilon}, u_{0}^{\epsilon}\right)$ satisfying (4.1) by using a strong compactness arguments. Indeed, it follows from (4.5) that $\left(n^{\epsilon, \delta}, c^{\epsilon, \delta}, u^{\epsilon, \delta}, \nabla c^{\epsilon, \delta}\right)$ is bounded uniformly in $L^{\infty}\left(0, \widetilde{T}_{2} ; H_{c o}^{m}\right)$ while $\left(\nabla n^{\epsilon, \delta}, \nabla u^{\epsilon, \delta}, \partial_{t} \nabla c^{\epsilon, \delta}\right)$ is bounded uniformly in $L^{\infty}\left(0, \widetilde{T}_{2} ; H_{c o}^{m-1}\right), \partial_{t} \nabla n^{\epsilon, \delta}$ is bounded uniformly in $L^{\infty}\left(0, \widetilde{T}_{2} ; H_{c o}^{m-2}\right),\left(\Delta n^{\epsilon, \delta}, \Delta c^{\epsilon, \delta}\right)$ bounded uniformly in $L^{\infty}\left(0, \widetilde{T}_{2} ; H_{c o}^{m-1}\right)$, and $\left(\partial_{t} n^{\epsilon, \delta}, \partial_{t} c^{\epsilon, \delta}, \partial_{t} u^{\epsilon, \delta}\right)$ bounded uniformly in $L^{\infty}\left(0, \widetilde{T}_{2} ; H_{c o}^{m-1}\right)$. Then, we obtain that $\left(n^{\epsilon, \delta}, c^{\epsilon, \delta}, u^{\epsilon, \delta}, \nabla c^{\epsilon, \delta}\right)$ is compact in $\mathcal{C}\left(0, \widetilde{T}_{2} ; H_{c o}^{m-1}\right)$ and $\nabla n^{\epsilon, \delta}$ compact in $\mathcal{C}\left(0, \widetilde{T}_{2} ; H_{c o}^{m-2}\right)$ by using the strong compactness argument. In particular, there exists a sequence $\delta_{k} \rightarrow 0^{+},\left(n^{\epsilon}, c^{\epsilon}, u^{\epsilon}, \nabla c^{\epsilon}\right) \in \mathcal{C}\left(0, \widetilde{T}_{2} ; H_{c o}^{m-1}\right)$ and $\nabla n^{\epsilon} \in \mathcal{C}\left(0, \widetilde{T}_{2} ; H_{c o}^{m-2}\right)$ such that

$$
\begin{aligned}
& \nabla n^{\epsilon, \delta_{k}} \rightarrow \nabla n^{\epsilon} \quad \text { in } \mathcal{C}\left(0, \widetilde{T}_{2} ; H_{c o}^{m-2}\right) \quad \text { as } \quad \delta_{k} \rightarrow 0^{+}, \\
& \left(n^{\epsilon, \delta_{k}}, c^{\epsilon, \delta_{k}}, u^{\epsilon, \delta_{k}}, \nabla c^{\epsilon, \delta_{k}}\right) \rightarrow\left(n^{\epsilon}, c^{\epsilon}, u^{\epsilon}, \nabla c^{\epsilon}\right) \quad \text { in } \quad \mathcal{C}\left(0, \widetilde{T}_{2} ; H_{c o}^{m-1}\right) \quad \text { as } \quad \delta_{k} \rightarrow 0^{+} .
\end{aligned}
$$

Moreover, applying the lower semi-continuity of norms to (4.5), we obtain the bounds (4.5) for $\left(n^{\epsilon}, c^{\epsilon}, u^{\epsilon}\right)$. In view of (4.5) and Lemma 2.3, we have that

$$
\sup _{0 \leq \tau \leq \widetilde{T}_{2}}\left\|\left(n^{\epsilon, \delta_{k}}, c^{\epsilon, \delta_{k}}, u^{\epsilon, \delta_{k}}, \nabla n^{\epsilon, \delta_{k}}, \nabla c^{\epsilon, \delta_{k}}\right)-\left(n^{\epsilon}, c^{\epsilon}, u^{\epsilon}, \nabla n^{\epsilon}, \nabla c^{\epsilon}\right)\right\|_{L^{\infty}} \rightarrow 0 .
$$

Hence, it is easy to deduce that $\left(n^{\epsilon}, c^{\epsilon}, u^{\epsilon}\right)$ is a weak solution of the chemotaxisNavier-Stokes equations. The uniqueness of the solution $\left(n^{\epsilon}, c^{\epsilon}, u^{\epsilon}\right)$ comes directly from the Lipschitz regularity of solution. Therefore, the whole family $\left(n^{\epsilon, \delta}, c^{\epsilon, \delta}, u^{\epsilon, \delta}\right)$ converges to $\left(n^{\epsilon}, c^{\epsilon}, u^{\epsilon}\right)$. Taking $\widetilde{T}_{0}=\widetilde{T}_{2}$ and $\widetilde{D}_{1}=\widetilde{D}_{4}$, we complete the proof of Theorem 1.1 . 


\section{The Proof of Theorem 1.2}

We can use the compactness argument that is almost the same as the one needed for the proof of Theorem 1.1 to prove Theorem 1.2. Hence we omit the details here.

Acknowledgements: I am very grateful to Professor Fucai Li for his valuable suggestions and encouragement during preparing this paper. This paper is supported by NSFC (Grant No.11271184).

\section{REFERENCES}

[1] A. Lorz, Coupled chemotaxis fluid model, Math. Models Methods Appl. Sci. 20 (2010) 9871004.

[2] L. C. Berselli, S. Spirito, On the vanishing viscosity limit of 3D Navier-Stokes equations under slip boundary conditions in general domains. Comm. Math. Phys. 316 (2012), no. 1, 171-198.

[3] J.-Y Chemin, Perfect incompressible fluids. Oxford Lecture Series in Mathematics and its Applications, Vol. 14. TheClarendon PressOxfordUniversity Press, NewYork, 1998.

[4] B. Franck, F. Pierre, Mathematical tools for the study of the incompressible Navier-Stokes equations and related models. Applied Mathematical Sciences, 183. Springer, New York, 2013.

[5] J. S. Fan, K. Zhao, Global dynamics of a coupled chemotaxis-fluid model on bounded domains. J. Math. Fluid Mech. 16 (2014), no. 2, 351-364.

[6] O. Guès, Problme mixte hyperbolique quasi-linaire caractristique. Comm. Partial Differential Equations. 15 (1990), no. 5, 595-645.

[7] J C. Gao, Q. Tao, Z. A. Yao, Strong solutions to the density-dependent incompressible nematic liquid crystal flows. J. Differential Equations. 260 (2016), no. 4, 3691-3748.

[8] H. Beirão da Veiga, F. Crispo, Concerning the $W^{k, p}$-inviscid limit for 3-D flows under a slip boundary condition. J. Math. Fluid Mech. 13 (2011), no. 1, 117-135.

[9] H. Beirão da Veiga, Vorticity and regularity for flows under the Navier boundary condition. Commun. Pure Appl. Anal. 5 (2006), no. 4, 907-918.

[10] H. Beirão da Veiga, F. Crispo, Sharp inviscid limit results under Navier type boundary conditions. An $L^{p}$ theory. J. Math. Fluid Mech. 12 (2010), no. 3, 397-411.

[11] D. Iftimie, F. Sueur, Viscous boundary layers for the Navier-Stokes equations with the Navier slip conditions. Arch. Ration. Mech. Anal. 199 (2011), no. 1, 145-175.

[12] J. G. Liu, A. Lorz, A coupled chemotaxis-fluid model: Global existence, Ann. Inst. H. Poincar Anal. Non Linaire 28 (2011) 643-652.

[13] E. F. Keller, L. A. Segel, Initiation of slime mold aggregation viewed as an instability, J. Theor. Biol. 26 (1970) 399-415.

[14] T. Kato, Nonstationary flows of viscous and ideal fluids in $\mathbb{R}^{3}$. J. Funct. Anal. 9 (1972), 296-305.

[15] Y. Maekawa, On the inviscid limit problem of the vorticity equations for viscous incompressible flows in the half plane, Comm. Pure Appl. Math., 67 (2014), pp. 1045-1128.

[16] N. Masmoudi, Remarks about the inviscid limit of the Navier-Stokes system. Comm. Math. Phys. 270 (2007), no. 3, 777-788.

[17] M. Chae, K. Kang, J. Lee, Existence of smooth solutions to coupled Chemotaxis-fluid equations, Discrete Contin. Dyn. Syst. 33 (2013) 2271-2297.

[18] N. Masmoudi, F. Rousset, Uniform regularity for the Navier-Stokes equation with Navier boundary condition. Arch. Ration. Mech. Anal. 203 (2012), no. 2, 529-575.

[19] M. Winkler, Global large-data solutions in a chemotaxis-Navier-Stokes system modeling cellular swimming in fluid drops, Comm. Partial Differential Equations 37 (2012) 319C352. 
[20] C. L. M. H. Navier, Sur les lois de l'équilibre et du mouvement des corps élastiques. Mem. Acad. R. Sci. Inst. France 6 (1827), 369.

[21] O. A. Oleinik, V. N. Samokhin, Mathematical models in boundary layer theory. Applied Mathematics and Mathematical Computation, 15. Chapman \& Hall/CRC, Boca Raton, FL, 1999.

[22] Q. Zhang, On the inviscid limit of the three dimensional incompressible chemotaxis-NavierStokes equations. Nonlinear Anal. Real World Appl. 27 (2016), 70-79.

[23] Q. Zhang, X. X. Zheng, Global well-posedness for the two-dimensional incompressible chemotaxis-Navier-Stokes equations. SIAM J. Math. Anal. 46 (2014), no. 4, 3078-3105.

[24] R. J. Duan, A. Lorz, P. A. Markowich, Global solutions to the coupled chemotaxis-fluid equations, Comm. Partial Differential Equations 35 (2010) 1635-1673.

[25] S. Agmon, A. Douglis, L. Nirenberg, Estimates near the boundary for thr solutions of elliptic partial differential equations satisfying general boundary conditions, $I I$. Comm. Pure Appl. Math. 17 (1964) 35-92.

[26] M. Sammartino, R. E. Caflisch, Zero viscosity limit for analytic solutions of the Navier-Stokes equation on a half-space. I. Existence for Euler and Prandtl equations, Comm. Math. Phys., 192 (1998), 433-461.

[27] M. Sammartino, R. E. Caflisch, Zero viscosity limit for analytic solutions of the Navier-Stokes equation on a half-space. II. Construction of the Navier-Stokes solution, Comm. Math. Phys., 192 (1998), 463-491.

[28] H. S. G. Swann, The convergence with vanishing viscosity of nonstationary Navier-Stokes flow to ideal flow in $\mathbb{R}^{3}$. Trans. Amer. Math. Soc. 157 (1971) 373-397.

[29] I. Tuval, L. Cisneros, C. Dombrowski, C. W. Wolgemuth, J. O. Kessler and R. E. Goldstein, Bacterial swimming and oxygen transport near contact lines. PNSA, 102 (2005) 2277-2282.

[30] Y. Wang, Z. P. Xin, Y. Yong, Uniform regularity and vanishing viscosity limit for the compressible Navier-Stokes with general Navier-slip boundary conditions in three-dimensional domains. SIAM J. Math. Anal. 47 (2015), no. 6, 4123-4191.

[31] Y. G. Cho, H. J. Choe, H.Kim, Unique solvability of the initial boundary value problems for the compressible viscous fluids. J. Math. Pures Appl. 83 (2004), no. 2,243-275.

[32] Y. L. Xiao, Z. P. Xin, On the vanishing viscosity limit for the 3D Navier-Stokes equations with a slip boundary condition. Comm. Pure Appl. Math. 60 (2007), no. 7, 1027-1055.

Department of Mathematics, Nanjing University, Nanjing 210093, P.R. China

E-mail address: zhpzhp@aliyun.com 